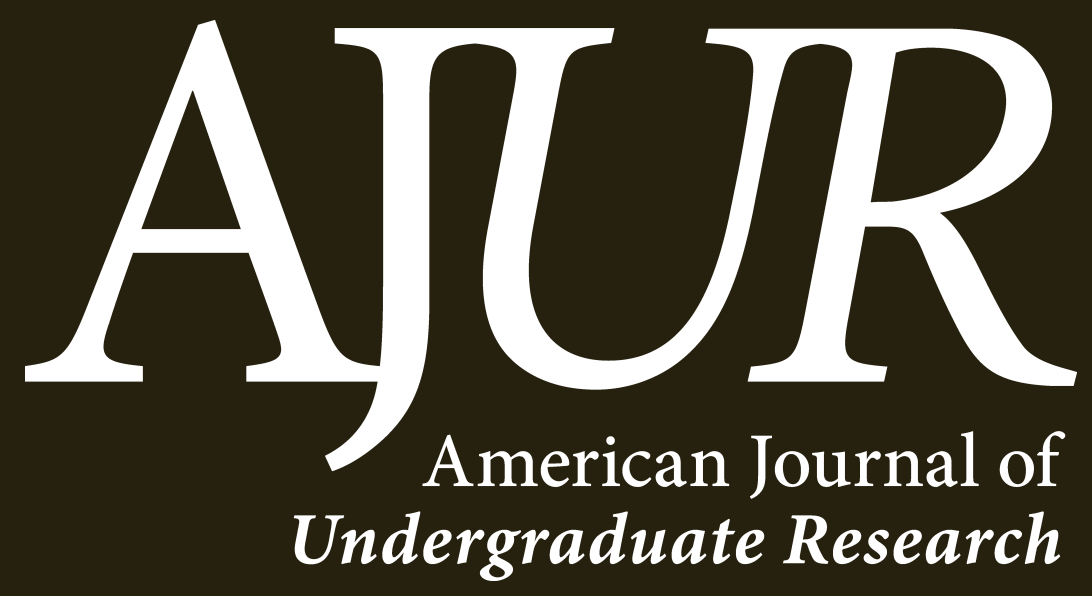

Volume 12 | Issue 3 | May 2015 www.ajuronline.org 


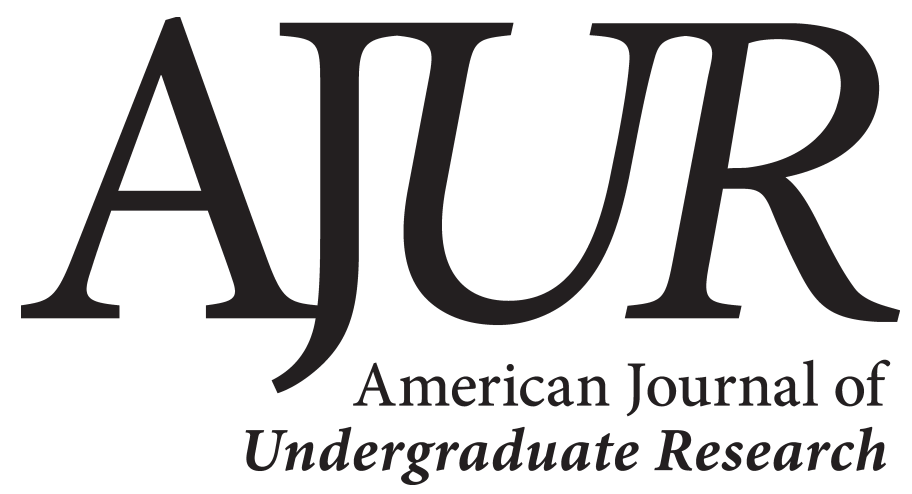

Volume 12 | Issue 3 | May 2015

$w w w . a j u r o n l i n e . o r g$

Editorial: Citizen Science: Contribute to a Common Good, Collaborate, and Communicate!

Special Thanks to AJUR's Sponsors

AJUR History and Editorial Board

Investigating the Response of a Small Urbanized

Watershed to Acute Toxicity Events via Analysis of

High-frequency Environmental Data

Holly Adelle Clark, Walter M. McDonald, Vinod K. Lohani \& Randel L. Dymond

Occurrence of Multiple Antibiotic Resistant Bacteria

in Aquatic Environments in Central Minnesota

Megan Bollin, Dr. Ellen Jensen \& Dr. David Mitchell

Effect of a Worksite Walking Competition on Health-Related Quality of Life Among University Employees

Breanna Z. Orozco, Lisa J. Leininger \& Kendra L. Contente

Approximation of Euler Number Using Gamma Function

Shekh Mohammed Zahid \& Dr. Prasanta Kumar Ray

Direct and Indirect Effects of Pseudoephedrine on the Intrinsic

Conduction System of the Embryonic Chicken Heart

Samely Gonzalez, Fatima Afzal \& Dr. Jacqueline S. McLaughlin

Nutritional Stress of Cultured Vero Cells Causes Altered Growth and Morphology as Seen in Neoplastic Transformation

Tyler Adams, Rabia Anwar, Michael Mfarej, Taylor Rundatz, Melissa Coyle \& Dr. Jacqueline S. McLaughlin

Searching for Gravitational Waves from the Coalescence of High-mass Black Hole Binaries

Liting Xiao, Alan J. Weinstein, Dr. Tjonnie G. F. Li \& Surabhi Sachdev 


\section{E D I T O R I A L}

\section{Citizen Science: Contribute to a Common Good, Collaborate, and Communicate!}

Would you like to have unlimited access to be engaged in a variety of research projects of your own choice? - Would you like your undergraduate research to contribute to a significant public health, medical, environmental, or other socially significant cause? - Would you like your data to be publicly available on an open database that is well curated and maintained? - Would you like to be engaged in research throughout the calendar year?

Citizen Science (www.citizenscienceassociation.org) engages diverse participants in thousands of research projects on local, regional, national, and global scales in the collection, interpretation, and use of scientific data on a particular scientific problem. Many examples empower the participants to engage in decision making about major issues such as: loss of biodiversity; evolutionary resistance to antibiotics, pesticides, herbicides, and cancer chemotherapy; identification of cancer clusters; monitoring effects of global warming and pollution into streams and ground water; spread of invasive species; folding proteins for possible development of HIV/AIDS drugs; and, identification of new pulsars, quasars, and stars with earth-like planets.

In a previous essay, I collaborated with undergraduate editors of the Journal of Young Investigators to assert the importance of undergraduates being authors, reviewers, and editors rather than just serving the interests of their advisors. ${ }^{5}$ AJUR has been an important voice in this conversation as it continues to publish vetted undergraduate authored research papers.

Too often, access to doing research as an undergraduate has been sorely limited to primarily rising seniors with high grade point averages, limited availability of funding, restrictions to prestigious research universities or private colleges, etc. Furthermore, because of decreased federal funding for research, too often many research projects serve corporate rather than societal needs. ${ }^{2}$ With the advent of new research efforts in participatory action research (PAR), crowdsourcing, open access, do it yourself (DIY) science, Maker Spaces, etc., numerous opportunities exist for anyone interested in a particular area to become engaged in a project where the data that you collect will be of interest to an external professional community.

You can choose from a broad range of citizen science: (1) "crowdsourcing — citizens as sensors" (e.g., contributing your labor or access to your computer); (2) "distributed intelligence — citizens as basic interpreters" (ranges from finding pulsars in telescopic images to solving protein folding problems or improving multiple sequence alignments); (3) "participatory science - participation in problem definition and data collection"; and, (4) "co-created" - ("designed by scientists and members of the public working together and for which at least some of the public participants are actively involved in most or all aspects of the research process") - "extreme - collaborative science - problem definition, data collection and analysis".', 6,7

As more students engage in a variety of citizen science projects, some core concepts of research that will be explored are: risk, uncertainty, expertise, transparency, credibility, trust, deliberation, complexity, participatory democracy, deliberative polling; citizens' juries, consensus conferences, foresight exercises, science shops, and citizenship. By engaging in citizen science, more students will also be introduced to the field of Science and Technology Studies (STS) to address such questions as: How do information, science, policy, and governance come together? How can students play an important role in deciding, observing and contesting how science and technology are being developed and implemented for the good of humanity and the natural world?

\section{— John R. Jungck, Senior Editor, AJUR Bioinformatics}

[1] Arnstein, Sherry R. (1969). A Ladder of Citizen Participation. Journal of the American Planning Association 35: 4 ,

[2] Bencze, Larry, and Lyn Carter. (2011). Globalizing Students Acting for the Common Good. Journal of Research in Science Teaching (Special Issue: Globalization-Science Education from an International Perspective) 48 (6): 648-669.

[3] Haklay, Muki. (2013). Citizen Science and Volunteered Geographic Information: Overview and Typology of Participation. In Daniel Sui, Sarah Elwood, and Michael Goodchild, Editors, Crowdsourcing Geographic Knowledge: Volunteered Geographic Information (VGI) in Theory and Practice, Springer, pp 105-122.

[4] Jordan, Rebecca, Alycia Crall, Steven Gray, Tina Phillips, and David Mellor. (2015). Citizen Science as a Distinct Field of Inquiry. BioScience 65 (2): 208-211.

[5] Jungck, John R., Margaret Harris, Renée Mercuri, and Joshua Tusin. (2004). Undergraduates: Do Research, Publish! CBE Life Science Education 3 (1): 24-26.
Director, Dupont Interdisciplinary Science Learning Laboratories Professor of Biological Sciences and Mathematical Sciences Affiliated Faculty: Computational Biology and Bioinformatics; Delaware Environmental Institute, University of Delaware

[6] Shirk, J., H. Ballard, C. Wilderman, T. Phillips, A. Wiggins, R. Jordan, E. McCallie, M. Minarchek, B. Lewenstein, M. Krasny, and R. Bonney, (2012). Public Participation in Scientific Research: A Framework for Deliberate Design. Ecology \& Society 17(2): 29 (20 pages).

[7] Wiggins, Andrea, and Kevin Crowston. 2010. Developing a conceptual model of virtual organisations for citizen science. International Journal of Organisational Design and Engineering 1(1/2): 148-162. 


\section{SPECIAL THA N KS}

AJUR is made possible through the assistance of our sponsors.

Support for this issue has been provided by the Office of the Provost, at the State University of New York at Oswego.

Thank you!

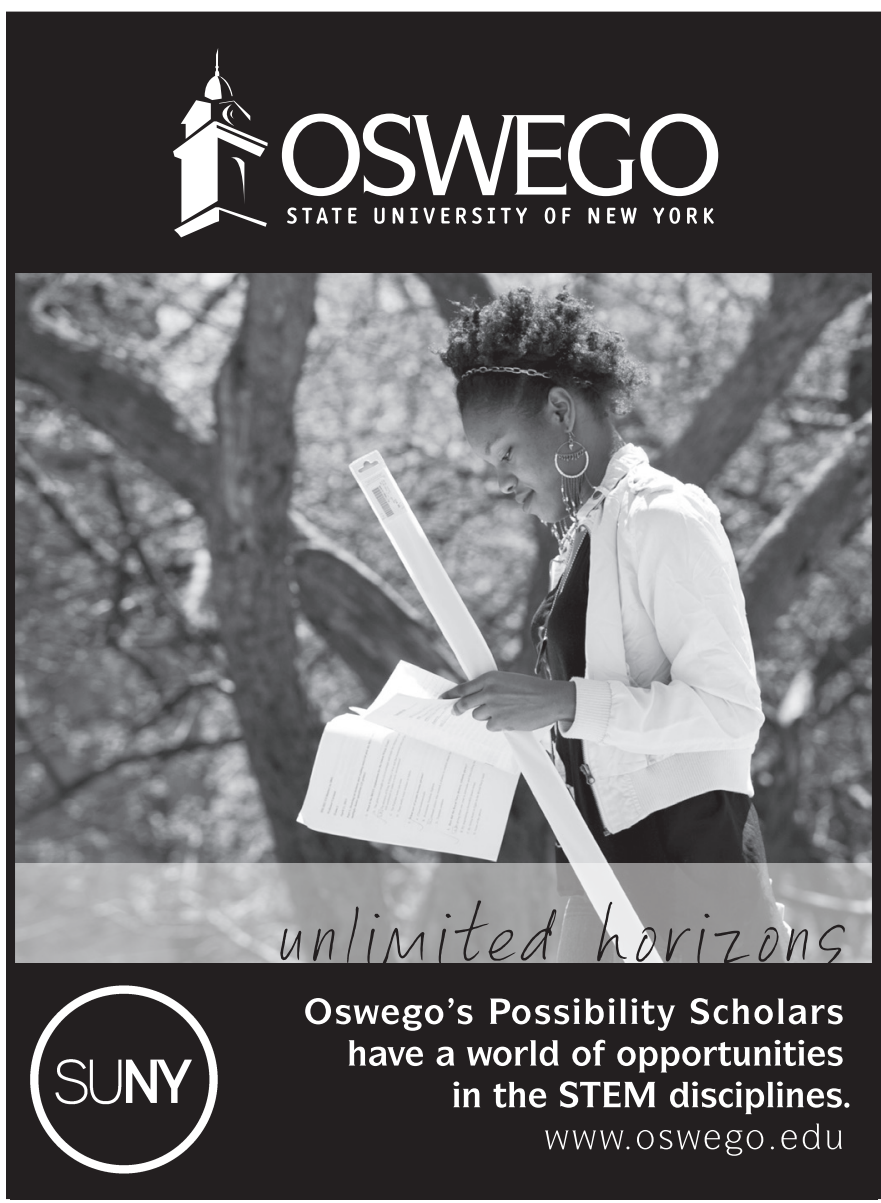

Interested in supporting quality undergraduate research? Request sponsorship information at editor@ajuronline.org 
American Journal of Undergraduate Research (AJUR) is a national, independent, peer reviewed, opensource, just-in-time, multidisciplinary student research journal for excellent student articles.

AJUR (ISSN 1536-4585) was established in 2002 by Dr. Cliff Chancey of the University of Northern Iowa, a true champion of undergraduate research. "Dr. Chancey's perspective was that the importance of peerreviewed research articles authored by undergraduates could not be overstated". Dr. Carl N. Drummond and Ms. Cathleen M. Carosella of Indiana University-Purdue University stewarded AJUR between 2013 and April of 2014, at which time the current editorial team assumed its role. The ISSN for AJUR's online edition is 2375-8732. The print edition remains ISSN 1536-4585.

\section{EDITORIAL TEAM Volume 12 | Issue 3 | May 2015}

Dr. Kestutis G. Bendinskas, Editor, editor@ajuronline.org

Dr. Anthony Contento, Assistant Technical Editor, technicaleditor@ajuronline.org

Rose Throop, Art and Copy Editor, copyeditor@ajuronline.org

Daniel Laird, Web Master,webmaster@ajuronline.org

EDITORIAL BOARD by subject area

ACCOUNTING
Dr. Dean Crawford,
dean.crawford@oswego.edu
ART HISTORY
Dr. Lisa Seppi,
lisa.seppi@oswego.edu
ASTROPHYSICS
Dr. Shashi Kanbur,
shashi.kanbur@oswego.edu
BEHAVIORAL NEUROSCIENCE
Dr. Aileen M. Bailey,
ambailey@smcm.edu
BIOCHEMISTRY
Dr. Pamela K. Kerrigan,
pamela.kerrigan@mountsaintvincent.edu
BIOENGINEERING
Dr. Jorge I. Rodriguez,
jorger@clemson.edu
BIOINFORMATICS
Dr. Kevin Daimi,
daimikj@udmercy.edu
Dr. John R. Jungck,
jungck@udel.edu
BIOLOGY, PHYSIOLOGY
Dr. David Dunn,
david.dunn@oswego.ed
BIOLOGY, DEVELOPMENTAL
Dr. Poongodi Geetha-Loganathan,
p.geethaloganathan@oswego.edu
BIOLOGY, MICROBIOLOGY
Dr. Peter Newell,
peter.newell@oswego.edu
BOTANY
Dr.William R. Bromer,
Dr. Julien Bachelier,
Dier@oswego.edu

\section{CHEMISTRY}

Dr Alfredo Castro, castroa@felician.edu

Dr. Charles Kriley,

cekriley@gcc.edu

Dr. Douglas Mulford, douglas.mulford@emory.edu

Dr. Mark A. Benvenuto,

benvenma@udmercy.edu

\section{CHEMISTRY, PHYSICAL}

Dr. John Oakes,

john.oakes@gcccd.net

\section{COMPUTATIONAL SCIENCES}

Dr. Dele Oluwade,

deleoluwade@yahoo.com

Dr. J. Ben Schafer,

schafer@cs.uni.edu

\section{ECOLOGY}

Dr. William R. Bromer, wbromer@stfrancis.edu

\section{EDUCATION, PHYSICS}

Dr. Andrew D. Gavrin,

agavrin@iupui.edu

\section{ENGINEERING, ENVIRONMENTAL}

Dr. Eileen M. Cashman,

eileen.cashman@humboldt.edu

\section{ENVIRONMENTAL SCIENCE}

Dr. Eileen M. Cashman,

eileen.cashman@humboldt.edu

\section{GEOLOGY}

Dr. Larry Davis,

ldavis@csbsju.edu

\section{HONORARY EDITORIAL}

BOARD MEMBER

Dr. Lorrie Clemo,

lorrie.clemo@oswego.edu

\section{KINESIOLOGY / EXERCISE SCIENCE}

Dr. David Senchina,

david.senchina@drake.edu

\section{LITERARY STUDIES}

Dr. Douglas Guerra,

douglas.guerra@oswego.edu

\section{MATHEMATICS}

Dr. Nkechi M. Agwu, nagwu@bmcc.cuny.edu

Dr. John Emert, emert@bsu.edu

Dr. Jeffrey J. Boats,

boatsjj@udmercy.edu

Dr. J.D. Phillips,

jophilli@nmu.edu

Dr. Dele Oluwade,

deleoluwade@yahoo.com

METEOROLOGY

Dr. Steven Skubis,

steven.skubis@oswego.edu

NEUROSCIENCE

Dr. Pamela E. Scott-Johnson, pamela.scottjohnson@morgan.edu

Dr. Amy Overman,

aoverman@elon.edu

PHYSICS

Dr. Carolina Ilie, carolina.ilie@oswego.edu

\section{PSYCHOLOGY}

Dr. Amy Overman, aoverman@elon.edu

Dr. Pamela E. Scott-Johnson,

pamela.scottjohnson@morgan.edu

STATISTICS

Dr. Mark Ecker, mark.ecker@uni.edu

TECHNOLOGY, ENGINEERING

Dr. Recayi Pecen,

regpecen@na.edu 


\title{
Investigating the Response of a Small Urbanized Watershed to Acute Toxicity Events via Analysis of High-frequency Environmental Data
}

\author{
Holly Adelle Clark ${ }^{a \star}$, Walter M. McDonald ${ }^{b}$, Vinod K. Lohani ${ }^{c}$, and Randel L. Dymond \\ ${ }^{a}$ Environmental Science, University of Idaho, Moscow, ID \\ ${ }^{b}$ Civil \& Environmental Engineering, Virginia Tech, Blacksburg, VA \\ ${ }^{c}$ Engineering Education, Virginia Tech, Blacksburg, VA \\ *Students: clar3176@vandals.uidaho.edu,walmcdon@vt.edu, \\ Mentors:vlohani@vt.edu,dymond@vt.edu
}

\begin{abstract}
This study, conducted over 10 weeks by an undergraduate student in an NSF REU (U.S. National Science Foundation Research Experiences for Undergraduates) program under mentorship of the co-authors, investigates responses of a small urban watershed, located in Blacksburg, Virginia, to acute toxicity events. High-frequency water and weather data were collected using monitoring equipment maintained by the Learning Enhanced Watershed Assessment System (LEWAS) lab, including a weather station, rain gauge, water quality sonde, and an Acoustic Doppler Current Profiler. The location and attributes of local stormwater catchments were surveyed and recorded using GIS software to determine the overall contributing area of the LEWAS lab watershed. This fieldwork resulted in the construction of an accurate stormwater network map, which in turn allowed the identification of sources of sedimentation and other pollution from runoff events. A case study presents the response of the watershed to a winter storm event that resulted in acute chloride toxicity from runoff containing road salts.
\end{abstract}

\section{KEYWORDS}

Water; Stormwater, Urban Watersheds; Watershed Responses; Acute Toxicity; High-frequency Data; Data Analysis; Data Collection; Environmental Monitoring; Flow Measurements; Hydrologic Data.

\section{INTRODUCTION}

Stroubles Creek flows approximately nine miles from Blacksburg, Virginia to the New River, and is classified as a second-order stream within the New River Watershed. Since the establishment of communities in the region beginning in 1740, the riparian health of the Stroubles Creek Watershed has fluctuated dramatically.' The major land use changes that have occurred as a result of settlement over the past 270 years have impacted the watershed's capacity to endure the ecological consequences of human activity. The Stroubles Creek Watershed has a history of impairment, as defined by the U.S. Environmental Protection Agency (EPA). In 1996, Stroubles Creek was found to be benthically impaired, and TMDL studies in 2003 revealed that sedimentation was a major cause of these impairments. ${ }^{2,3}$ In the past decade, restoration efforts have been implemented to help restore the riparian health of the Stroubles Creek Watershed. Figure 1 illustrates the location of the watershed including the land use distribution and impaired reach of Stroubles Creek. 




Figure 1. Stroubles Creek watershed land use and LEWAS lab location.

The Learning Enhanced Watershed Assessment System (LEWAS) lab is located on the Virginia Tech (VT) campus in the Webb Branch tributary $\left(2.78 \mathrm{~km}^{2}\right)$ of Stroubles Creek $\left(25 \mathrm{~km}^{2}\right)$. The watershed is highly urbanized and subject to flash flood events and urban stormwater pollutants. The lab operates multiple in-situ water and weather monitoring sensors for the purpose of water sustainability research, education, and public outreach. These sensors collect flow, water quality, and weather parameters in high-frequency temporal intervals $(0.1-3 \mathrm{~min})$ which allow the site to capture ephemeral responses of the watershed to flash events. The LEWAS lab aims to utilize high-frequency data collected in real-time to gain insight into the acute responses of a small urbanized watershed.

The flow and water quality at the LEWAS site is highly sensitive to the impacts of urban disturbances and pollutants. The watershed has been heavily developed over time (with approximately $30 \%$ of the watershed covered with impervious surfaces) and is prone to channeling large amounts of runoff during precipitation events due to the large area of impermeable surfaces. This runoff can also increase the amount of sediment that is displaced, resulting in erosion and excess sediment deposition. In addition, the runoff introduces pollutants into the aquatic system in the form of chemical compounds, trash, and debris. The type of pollutants present in the runoff is dependent upon climatological, physical, and spatial characteristics of the watershed. For instance, many studies suggest that deicing salts administered in urban areas prone to freezing temperature pose a threat to water quality in terms of chloride toxicity. ${ }^{4,5}$ More rural agricultural communities, such as those closer to the outlet of the Stroubles Creek watershed, may have other factors that might be of concern such as pesticides and fertilizers, which can contain high levels of nutrients, such as nitrates and phosphates. In excess, these compounds can cause eutrophication and can impair the ecosystem. Stroubles Creek's history of impairment and the increased urbanization in Blacksburg makes water quality and quantity monitoring an important tool for understanding causes of impairment.

The LEWAS lab hosts a National Science Foundation Research Experiences for Undergraduates (NSF/REU) Site on Interdisciplinary Water Sciences and Engineering. The goal of this site is to engage qualified undergraduate researchers drawn from various academic institutions into a 10-week interdisciplinary research project in water sciences and engineering. Faculty and graduate students from various departments including Engineering Education, Civil \& Environmental Engineering, 
Biology, Geosciences, Crop \& Soil Environmental Sciences, and Industrial Design mentor undergraduate researchers. To date, 66 researchers (39 women and 27 men) representing $50+$ institutions have graduated from this Site. This study was conducted by one of the REU students from the University of Idaho (lead author) over the summer of 2013. In order to achieve the goal of the study four objectives were established: 1) understand how a real-time, high-frequency monitoring system functions and how to address malfunctions of the components involved in order to maintain a functional monitoring system; 2) investigate sources and travel paths of sedimentation from flash runoff events; 3) delineate a stormwater network map of the Webb Branch watershed to verify the watershed network and area; and 4) investigate how deicing salts are transported through stormwater runoff to the field site and the impact this has on the water quality.

\section{LEWAS FIELD SITE}

The outdoor site of the LEWAS Lab was chosen due to the location on VT campus and the environmental significance of the creek. This site monitors one of two major inflows to a campus retention facility known as the "Duck Pond," which contains drainage from portions of the town of Blacksburg and VT campus. Over $95 \%$ of the land cover in the watershed is urbanized and thus covered with impermeable surfaces that slope (6.5\%) towards the watershed outlet at the LEWAS site. The urban runoff from the watershed flows through a four foot box culvert to a natural run of the stream where the LEWAS sensors are located and on into the Duck Pond. Consequentially, the water quality at this location is indicative of not only how anthropogenic activity impacts water quality entering the Duck Pond, but also of the watershed's functionality during flood events.

Water and weather parameters are measured and recorded by multiple in-situ sensors at the outdoor location of the LEWAS lab. The in-situ sensors include a Sontek Argonaut-SW Acoustic Doppler Current Profiler (ADCP) that is mounted on the streambed and measures stage and velocity every minute. Meteorological conditions at the site are recorded with Vaisala Weather Station equipped with sensors capable of measuring air temperature, pressure, and relative humidity every one minute, wind speed and direction every five seconds, and precipitation instantaneously using a piezoelectric sensor that detects the impact of individual raindrops. Water quality parameters including temperature, $\mathrm{pH}$, turbidity, specific conductance, dissolved oxygen, oxidation reduction potential, percentage of saturated dissolved oxygen and total dissolved solids are measured with a Hach MS5 Hydrolab Sonde and collected every three minutes. A tipping-bucket rain gauge has also been installed at the site to verify and calibrate precipitation readings from the weather station. An ultrasonic transducer has been installed to provide an additional flow measurement through stage readings behind a weir located in the culvert upstream of the site. A diagram of the physical layout of the field site is illustrated in Figure 2.

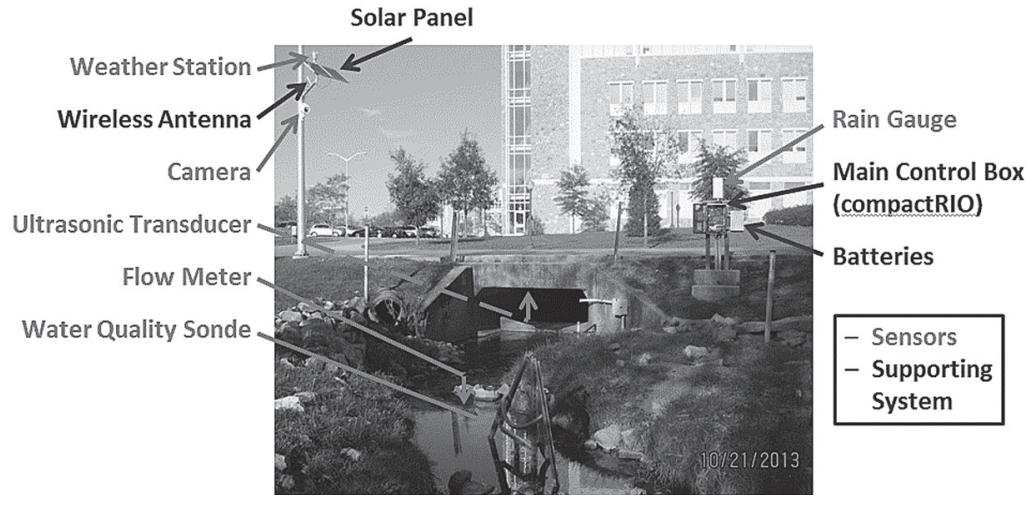

Figure 2. LEWAS lab field site on Stroubles Creek. 
Data from the sensors is collected using an embedded computer located in the main control box that runs LabVIEW software and transmits data through the campus wireless network to a database where it is accessed through a live data viewing website (http://www.lewas.centers.vt.edu/ dataviewer) ${ }^{6}$. Through the website, the LEWAS lab provides public access to environmental data captured by the sensors in order to increase educational outreach and public awareness of the impact that urbanization has on a local watershed. In fact, as part of the educational outreach of the lab data from the sensors has been used to improve student learning in multiple courses at VT and Virginia Western Community College (VWCC). ${ }^{7,8}$

\section{RESEARCH METHODS}

\subsection{Maintenance \& Calibration}

The mechanics of a real-time, high-frequency monitoring system and the complications associated with environmental sensors are two fundamental components of a continuous hydrology lab. Accurate and valid data are only generated by sensors that are in good physical condition and that have undergone meticulous calibration. Routine maintenance tasks (physical cleaning, calibration, electrical rewiring, professional repairs, etc.) are fundamental to a functioning lab. Sustaining an operational monitoring system is a primary research objective in itself, for the collection and quality of any subsequent data is dependent upon the sensors' condition.

Due to the high volume of water that flows past the LEWAS site during significant precipitation events (Figure 3), frequent sonde and ADCP sensor maintenance and occasional repairs have to be performed. These repairs must be completed as efficiently as possible to reduce the amount of time that the site is unmonitored. Routine calibrations require the sensors to be removed from the stream, cleaned in a laboratory environment, and reset with standard solutions for each parameter at approximately three-week intervals. The frequency of calibration is partly determined by the drift in measurements that have been observed since the previous procedure; if an increased margin of error is noted, then calibrations will be performed more frequently.

During high flow events, debris in a variety of mediums (substantial rocks, urban garbage, vegetation, etc.) that has been channeled through the stormwater network arrives at the site and can potentially collide into the ADCP and sonde (Figure 3). This can cause erroneous measurements and sensor damage and disturb the riparian ecosystem by damaging existing habitat and increasing turbidity and other pollutants. Debris that accumulates around or within the sensors must be manually removed to maintain accurate data.

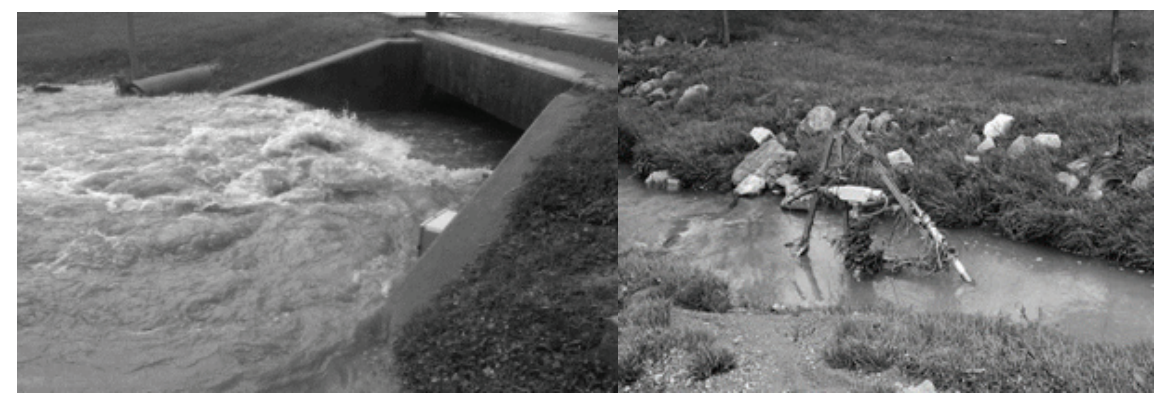

Figure 3. Flooding from rain event at LEWAS field site \& Hach MS5 Hydrolab water quality sonde covered in Debris; Sedimentation deposits on south stream bank. 
The following example describes how damage to a sensor results in repair, redeployment, and calibration efforts. In July 2013, the ADCP underwent a series of repairs following severe rain events to address internal electrical damage. The issue became apparent when measurements were not being recorded by the instrument, and it was deduced that the connection between the conduit and the instrument had been exposed to water for a prolonged period of time. This caused water to leak into the instrument, and the pins of the male connector to become corroded (Figure 4). The lack of a functional water-tight seal could have resulted from a number of situations including the accumulation of biofilm around the port preventing a flush seal, debris during storm events impacting the port and breaking the seal, or improper insertion of the cord resulting in a leak.

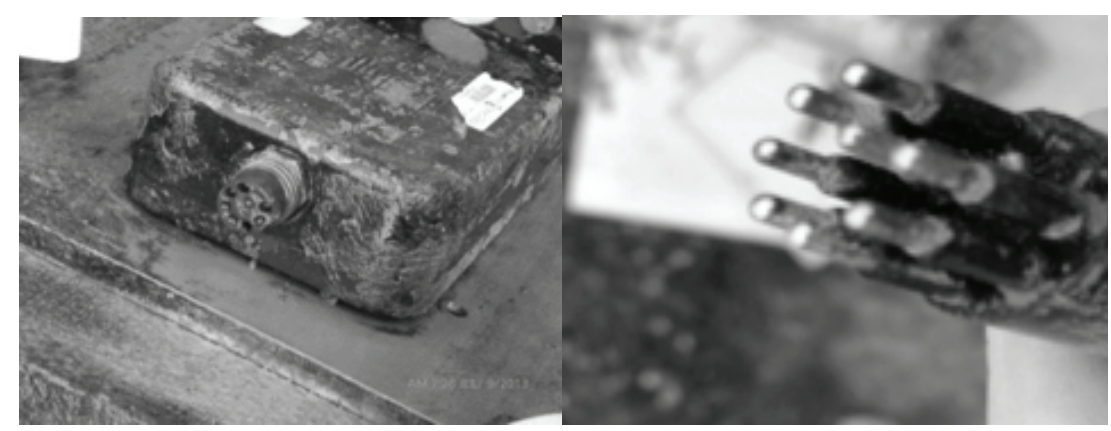

Figure 4. ADCP flow-meter with corroded biofilm accumulation and corroded conduit pins.

To repair the ADCP, it was removed from the streambed and the build-up of biofilm was removed with a poly-fiber brush. Electrically-conducting silicone spray was deposited into the female ports of the ADCP to create a water-tight seal. The damaged conduit wire containing the connector was spliced together with a replacement cord to achieve the necessary length using a soldering connection. The connector was then inserted into the ports of the instrument.

After the ADCP was returned to the streambed, the stage-area rating of the cross section was calibrated by conducting a new cross-section survey. The streambed is composed of cobbles and pebbles with alluvia-floodplain deposits of stratified unconsolidated silt, clay, and sand with lenses'. During a storm event, a stream's cross section can sometimes be altered dramatically due to erosion and sedimentation caused by the high volume and velocity of water passing through. The ADCP estimates flow based on the index velocity method ${ }^{9}$ which takes the velocity and stage readings measured by the device and applies them to index-velocity and stage-area ratings respectively. If the cross section of the stream changes due to sedimentation or erosion it will affect the stage-area rating and subsequently the volume of water estimated by the ADCP. A cross-sectional profile survey was performed at the site in two locations (at the ADCP and the calibration cross section) to generate the new cross section of the stream. The cross-sectional profiles were recorded using a Leica Total Station and were imported into Microsoft Excel to generate a new graphic cross section of the stream. Figure 5 represents the changes to the cross sectional profile of the stream during three separate surveys during the spring of 2012, spring of 2013, and summer of 2013. The surveys are aligned by a benchmark located along the left bank with the vertical datum representing the point of lowest elevation. 


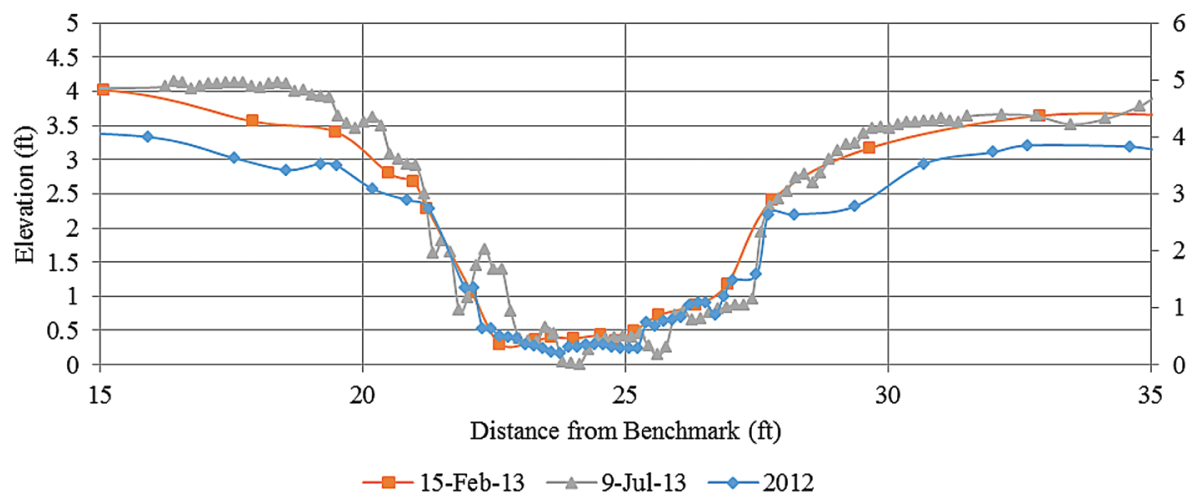

Figure 5. Seasonal comparisons of cross sections of Stroubles Creek.

A Sontek FlowTracker Handheld acoustic doppler velocimeter (ADV) point velocity instrument was used to validate the existing index velocity rating at the calibration cross section. The calibration cross section was chosen downstream of the ADCP cross section at a distance downstream of four to five times the width of the channel. The index velocity rating relates the mean velocity of the stream channel, computed through the ADV measurements, to the index velocity measured by the ADCP. Point velocities were recorded using the ADV along the stream cross section at various intervals to compute mean velocity. The mean velocity was then correlated with the index velocity measurement given by the ADCP to validate the existing index-velocity rating.

Maintaining an accurate index-velocity rating is important for valid velocity readings and water quantity computations, as well as for water quality concerns. The velocity in the stream is related to the size of sediment (and any debris or pollutant that it contains) that is physically able to be transported to the catchment location. Knowing the volume of water that is discharged from the LEWAS Lab field site allows projections to be made about the mass of sediment that is being deposited at the Duck Pond, and the mass (rather than concentration) of the measured pollutants passing the LEWAS field site.

\subsection{Sedimentation}

After ensuring accurate and reliable data from the environmental sensors, an investigation was conducted into the sources of pollutants captured by the sensors. The first pollutant of interest was the large amounts of sediment that are transported through the site during storm events. Substantial sediment deposition that occurs during high flow events at the field site has a negative impact on data accuracy, water quality, and riparian health. The impact of sedimentation on the water quality at the site is indirectly measured through turbidity and total dissolved solids (TDS) measurements collected by the sonde. Turbidity and TDS measure the optical determination of water clarity and the amount of material dissolved in water respectively, and may not be a good indicator of sediment transport is the sediment is coarse. High flow events that increase sedimentation repeatedly resulted in sensors of the sonde getting clogged with a combination of both large rocks and clay-like material and algae, resulting in inaccurate data. To clear the openings of the sensors, a plastic handled dish brush and cotton swabs were used to scrub off the sediment and biofilm after storm events.

An example of the sedimentation at the site is shown in Figure 6 which illustrates the sediment deposition that accumulated in a culvert located 10 meters upstream of the LEWAS in-stream sensors. The mounds of sediment shown in the figure demonstrate the amount of sediment that is routinely transported through the stormwater network and eventually into the Duck Pond. The Duck Pond has had a history of poor water quality, much of which is attributed to sediment. The 
pond functions as a large-scale stormwater catchment and was dredged three times in 1950, 1960, and 1986 because of detrimental sediment deposition. ${ }^{1}$

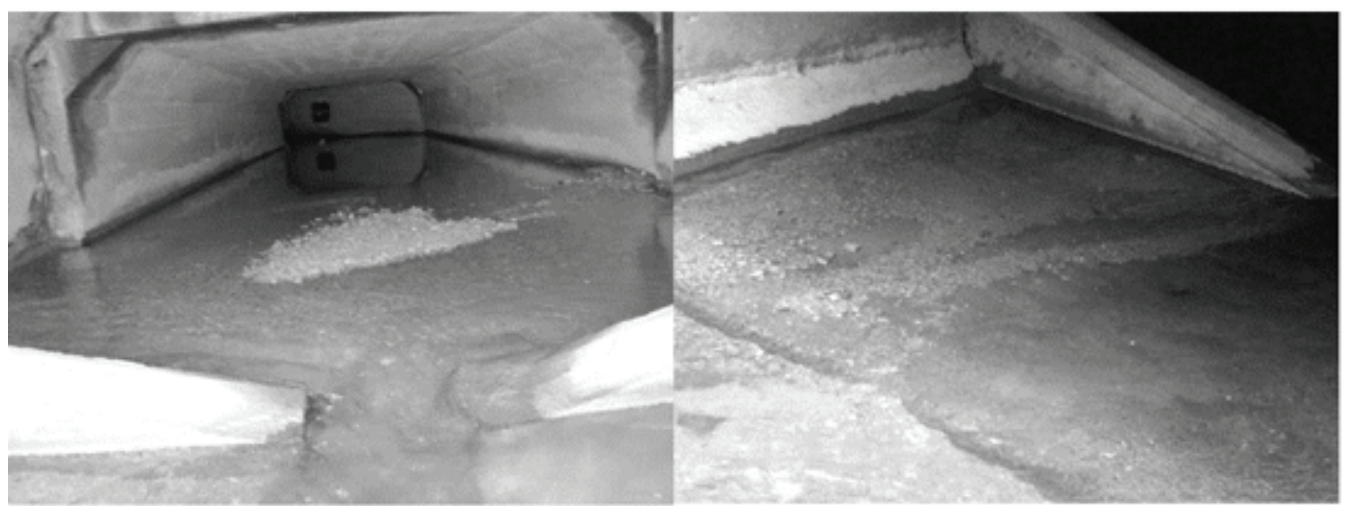

Figure 5. Sedimentation deposits in culvert and weir (looking upstream).

To investigate the sources of sedimentation from flash events that deposit large amounts of sediment near the site, a physical watershed investigation was conducted. Possible sources of sedimentation were documented through geo-tagged pictures during a summer storm event. Pictures captured various sources of sedimentation as well as travel paths of the sediment, such as small streams and stormwater inlets that were visibly transporting and storing sediment during the runoff period. These pictures were uploaded and georeferenced using GIS software to document the most likely sources of sedimentation (Figure 7). This information was combined with stormwater network data described in section 3.3 to determine leading sources of sedimentation.

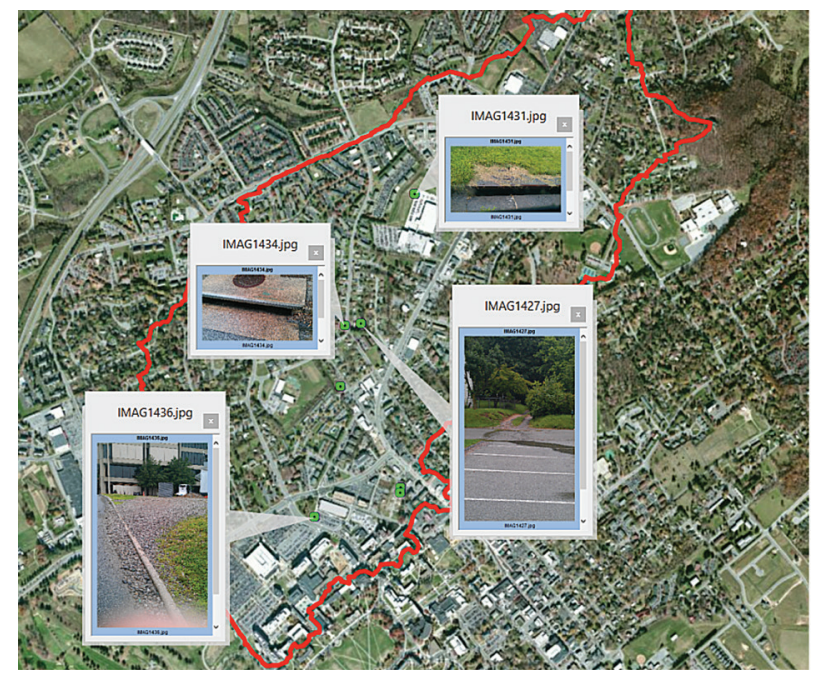

Figure 6. Sedimentation survey map.

\subsection{Stormwater Network Map}

To determine the path that sources of pollutants such as sediment take to get to the LEWAS site, a stormwater network survey was conducted in a portion of the McBryde neighborhood, a residential area just north of the Virginia Tech Campus and part of the contributing watershed. A map of the stormwater catchments and pipes for this portion of Blacksburg did not exist prior to this survey; 
however, much of the stormwater network for the LEWAS watershed had already been completed prior to June 2013. Understanding the stormwater network in this region is necessary to determine whether (and if so, how) runoff from the neighborhood reaches the field site. An understanding of the network will allow the routes of sediment loads and other pollutants to be understood, which is useful in eventually developing models that can predict pollutant loads for simulated events.

To begin constructing a stormwater network map, a geographic information system was used to locate catchments in the McBryde neighborhood that were incomplete and needed surveying. A marker was placed at each stormwater infrastructure feature, such as an inlet or outfall, to generate a fieldwork map of the locations to visit in person. While at each stormwater feature, measurements of its attributes (pipe material, diameter, shape, azimuth, depth, type of structure, etc.) were recorded into a handheld ArcPad device and pictures of the stormwater structures were taken with a GPS camera. The catchment locations and corresponding attributes stored on the handheld ArcPad were imported to ArcMap. Custom python scripts were executed within ArcMAP to create a connected stormwater network from the point shapefile imported from the $\mathrm{ArcPad}^{10}$. This created a completed stormwater network that allows investigators to connect the travel path of pollutants captured by the LEWAS sensors to their sources.

\subsection{Acute Chloride Toxicity}

The previous research methods allow the LEWAS site to effectively analyze events in the watershed that are captured by the LEWAS environmental sensors. The following case study illustrates how the LEWAS watershed responds to an acute chloride toxicity event from a spring storm occurring over a two day period. Blacksburg experienced a spring storm over March 24-25, 2013, as indicated by the meteorological data collected by the weather station illustrated in Figure 7 . The weather station detected a drop in air temperature and air pressure and a rise in rainfall intensity during the storm event.
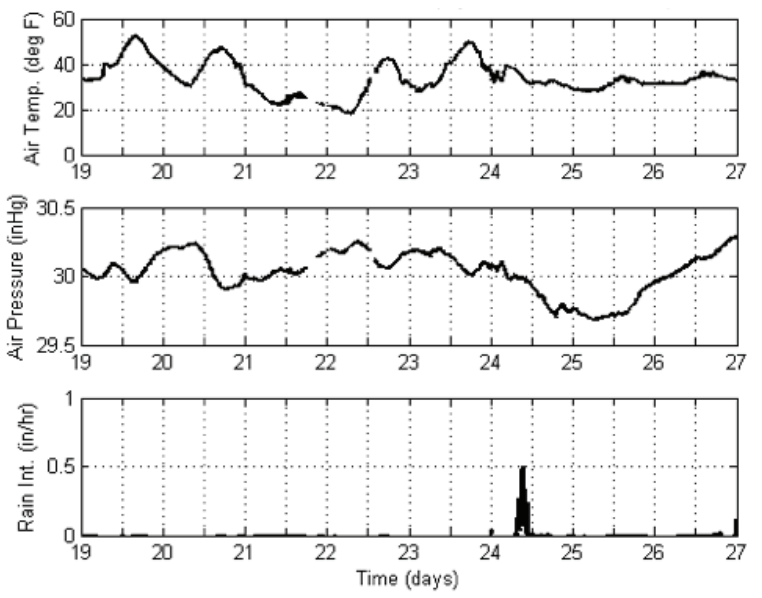

Figure 7. Weather station data for duration of March 24-25, 2013 storm event.

In addition, the water quality sonde captured a significant spike in specific conductance from runoff during the storm. Before this spring storm event, road crews applied road salts and deicing solution on the road and sidewalks in portions of the town of Blacksburg and Virginia Tech campus within the LEWAS watershed. Road salts contain a large amount of sodium chloride $(\mathrm{NaCl})$, which when added to water, dissolves into chloride $\left(\mathrm{Cl}^{-}\right)$. The Transportation Planning Engineer and Facilities Operations Director at Virginia Tech were contacted to confirm the chemical composition of the deicing compound Virginia Tech administers (M. Dunn \& M. Helms, personal 
communication, June 22, 2013). According to the Material Safety Data Sheet, the deicing compound is approximately $99 \% \mathrm{NaCl}$. This information is necessary to trace the potential sources of chloride from winter storm events. A high concentration of chloride in freshwater systems can have a negative impact on the aquatic health of the ecosystem."

Although chloride is not directly measured by the LEWAS system, specific conductance can be used as a surrogate measurement to estimate the concentration of chloride in the stream. A United States Geological Survey (USGS) study compared 24 stream locations that recorded specific conductance and chloride concentrations in Broad Run watershed in Fairfax County, Virginia and developed a general relationship when the specific conductance is over $1,000 \mu \mathrm{S} / \mathrm{cm}$, indicating heavy road salt content. ${ }^{12}$ This equation was applied to the specific conductance data collected at the LEWAS site:

$$
\mathrm{CC}=(0.333) \mathrm{SC}
$$

Equation 1.

where CC is the chloride concentration in $\mathrm{mg} / \mathrm{L}$, and SC is specific conductance amount in $\mu \mathrm{S} / \mathrm{cm}$. There are limitations to using Equation 1 as the relationship is not specific to the LEWAS site. However, given that this equation was developed in a similar watershed at various locations under similar conditions, it can give a general idea of the trends and possible chloride exceedances during a storm event at the site after a heavy road salt application.

\section{RESULTS AND DISCUSSION}

A significant portion of the McBryde neighborhood's stormwater conduits was successfully mapped out over the course of this summer investigation, thus improving the understanding of the contributing Upper Stroubles Creek watershed area. Figure 8 shows the portion of the McBryde area $\left(0.44 \mathrm{~km}^{2}\right)$ that was mapped as a result of this work in relation to the entire LEWAS watershed.

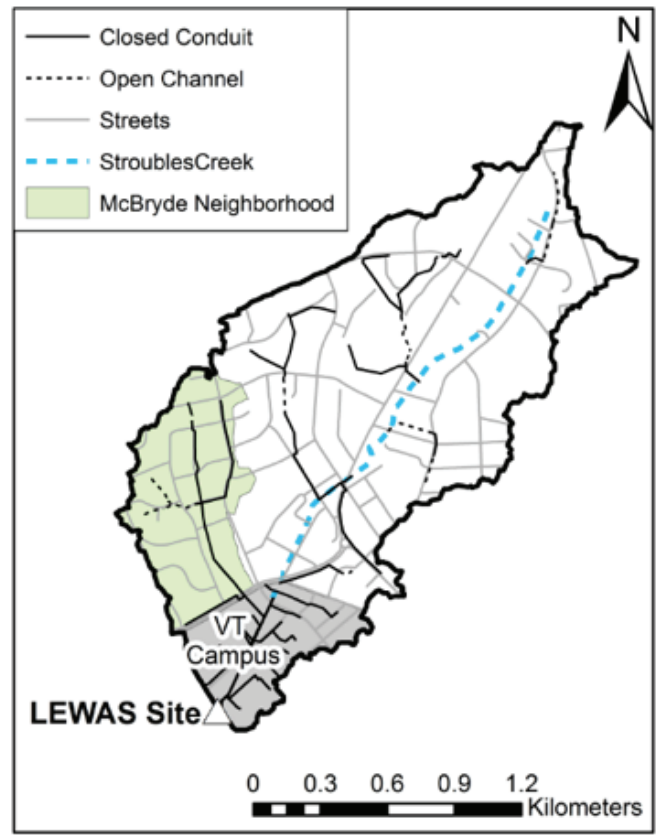

Figure 8. Portion of McBryde stormwater network system mapped during summer 2013. 
The stormwater network maps created as a result of the survey process ultimately provide a visual explanation of how pollutants and sediment arrive at the LEWAS lab field site. Knowing the path that a pollutant travels can allow estimates to be made of time that lapses between a pollutant entering a given conduit and its presence recorded by the sensors at the monitoring site. By using the stormwater network map in Figure 8 along with knowledge of the land cover, investigations into possible sources of pollution captured by the sensors can be conducted.

As an example, the stormwater network data along with the watershed sedimentation survey was combined to provide clues to the probable sources of sedimentation during flash storm events. The stormwater network map was used to properly delineate the watershed area, which provided the area of possible pollutant sources within the town. The geotagged photos within GIS were used to determine possible sedimentation source locations within the watershed and the stormwater network was used to determine the routes for each source. This information was finally used to map highly probable areas that contribute to sedimentation at the field site. The leading sources of sediment runoff within the watershed were identified as construction sites and gravel parking lots, many of which are adjacent to the main reach of Webb Branch. These sources were found to contribute sediment during flash storm events that cause spikes in turbidity and deposits of sediment at the site and in the Duck Pond, which will again require dredging and channel modifications over time. Identifying these sources can help lead to solutions by informing stormwater managers and decision makers about sedimentation impairments within Webb Branch watershed.

Results from a case study of a spring storm event suggest that there are substantial environmental consequences to the application of road salts and deicing solutions in the LEWAS watershed. The water quality sonde recorded multiple spikes in specific conductance at the field site during the March 24-25, 2013 storm event. Specific conductance represents the ability of water to conduct electrical current and can be used as a surrogate to approximate the amount of chloride in the water due to deicing solutions. Figure 9 shows the estimated chloride concentrations over the duration of the runoff event as well as the EPA recommended acute toxicity limit. Acute chloride toxicity is the potential exceedance of a species tolerance to chloride caused by a sudden, one time, and high exposure. The U.S. Environmental Protection Agency (EPA) sets recommended limits of pollutants that are toxic in acute exposures for various freshwater organisms. The EPA quantifies an acute toxicity level for chloride as $860 \mathrm{mg} / \mathrm{L}^{11}$. As illustrated, the chloride concentration exceeded this limit on multiple occasions, indicating that the system was experiencing an acute toxicity event. (Unfortunately, the ADCP was not functioning during this event so the LEWAS was only able to capture water quality data.)

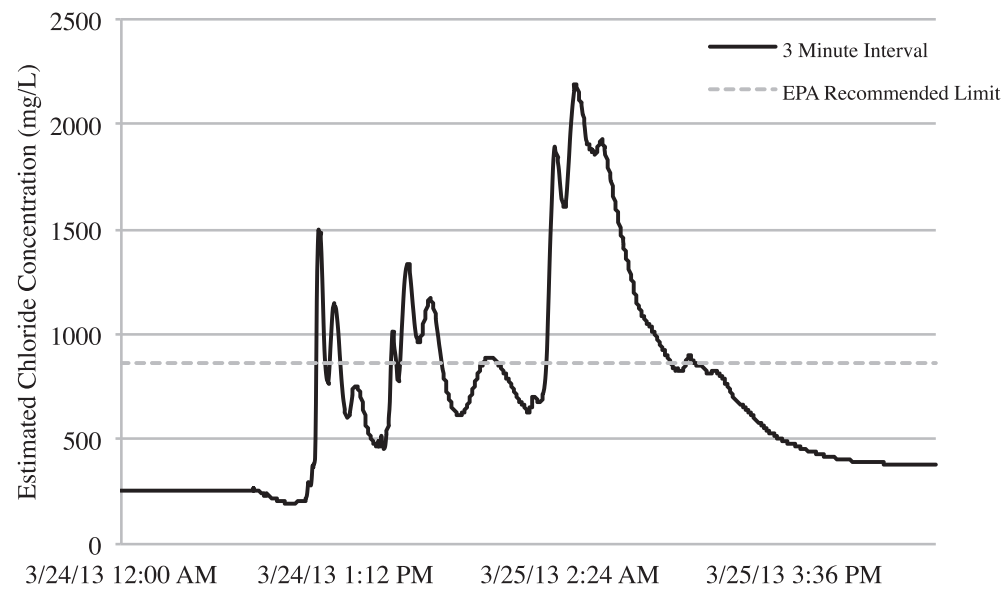

Figure 9. Measured chloride concentrations vs. EPA recommended concentration. 
In addition to the EPA recommended limit, the toxicity thresholds of key indicator species can be used to determine the impact of the chloride. A professor in the Virginia Tech Biology Department explained that the fathead minnow (Pimephales promelas) is frequently used as an indicator species by researchers to gauge the health of a riparian ecosystem (J. Webster, personal communication, July 10, 2014). The acute toxicity limit of a fathead minnow, as determined by the EPA, is $860-2790$ $\mathrm{mg} / \mathrm{L}^{11}$. Because the fathead minnow is frequently found in Stroubles Creek, these high chloride concentrations are of concern and suggest that this event most likely created an environment that was toxic to the fathead minnow.

The survey of the stormwater network and land cover of the watershed helps to determine the sources of chloride in the system. The proximity of the LEWAS Lab field site (and ultimately the Virginia Tech Duck Pond) to a main road is a contributing factor to the sudden spike in specific conductance, as there is very little distance between runoff that originates on the adjacent road where road salts are applied and the monitoring system, consequently eliminating the opportunity for natural ground filtration. The phenomenon of ground filtration is also diminished in a manmade conduit system, where the pipes are usually made of impermeable materials, such as corrugated plastic or concrete. Understanding the characteristics of a watershed's stormwater network system allows the likelihood of processes such as ground filtration to be determined; if the runoff does not have the opportunity to be channeled over land, it is likely to be more concentrated with pollutants and sediment.

The results of this study help to quantify the impact that deicing solutions and road salts have on the water quality entering the Duck Pond facility. The results suggest how an urban practice - the act of salting roads - can impact the local watershed. When freezing precipitation melts due to contact with road salts, the water carries these salts through the stormwater network and into the stream. When sodium chloride comes into contact with water it dissolves into chloride ions, which cannot be removed with structural Best Management Practices (BMPs). The only solution to improve water quality that is contaminated with high levels of chloride is to dilute it. Although human safety during winter storms is of the highest importance, decision makers can use this information to try to optimize the application of deicing solution and road salts in order to promote human safety while mitigating the impact to the environment.

\section{CONCLUSION}

During the 10-week NSF REU program, an undergraduate researcher had the opportunity to use real-time, high-frequency watershed monitoring data to analyze sedimentation and acute chloride toxicity from flash watershed events. The sensors that provide data in real-time require a great deal of maintenance, but providing reliable water and weather data in a riparian ecosystem is essential to understanding the relationship between a city and its watershed. This investigation of the LEWAS watershed is ongoing, and it is recommended that the following action items be addressed to continue this research.

- The sonde's sensors should be cleaned daily of debris and calibrated more frequently (every two weeks) to ensure that the sensors are not subject to repeat damage.

- A LEWAS Lab member should visit the field site during the winter months (especially following a snowstorm) to look for fish kills. This could strengthen the hypothesis that road deicing salts are creating a hostile environment for aquatic organisms.

- In addition, grab samples should be taken throughout a winter storm event and analyzed for chloride concentrations to develop a regression equation between specific conductance and chloride that is specific to the LEWAS site.

- A chronic chloride toxicity analysis should be performed during the winter to provide an idea of how the system responds to pollutants over the course of several days or weeks. 


\section{ACKNOWLEDGMENTS}

The authors acknowledge LEWAS lab students Daniel Brogan, Hari Raamanathan, and Arun Rai who are not included in authorship. We acknowledge the support of the National Science Foundation through NSF/REU Site Grant EEC-1062860. Any opinions, findings, and conclusions or recommendations expressed in this paper are those of the author(s) and do not necessarily reflect the views of the National Science Foundation.

\section{REFERENCES}

[1] Parece, T., DiBetitto, S., Sprague, T., and Younos, T. (2010) The Stroubles Creek Watershed: History of Development and Chronicles of Research, In Virginia Water Resources Research Center Special Report (Blacksburg, Ed.), Virginia Polytechnic Institute and State University.

[2] VEDQ. (2006) Upper Stroubles Creek Watershed TMDL Implementation Plan Montgomery County, Virginia, In VT-BSE Document Virginia Department of Environmental Quality VDEQ, Blacksburg, Virginia.

[3] VDEQ. (2012) 305(b)/303(d) Water Quality Assessment Integrated Report, Virginia Department of Environmental Quality (VDEQ), Richmond, VA.

[4] Corsi, S., Graczyk, D., Geis, S., Booth, N., and Richards, K. (2010) A Fresh Look at Road Salt: Aquatic Toxicity and Water-Quality Impacts on Local, Regional, and National Scales, Environmental Science \& Technology, 44, 7376-7382.

[5] SEWRPC. (2014) Acute Toxicity of Sodium Chloride to Freshwater Aquatic Organisms." (Appendix E), In A Watershed Restoration Plan for the Root River Watershed. SEWRPC Community Assistance Planning Report Southeastern Wisconsin Planning Commission (SEWRPC).

[6] Brogan, D., Lohani, V., and Dymond, R. (2014) Work in Progress: The Platform-Independent Remote Monitoring System (PIRMS) for Situating Users in the Field Virtually, In Proc. 2014 ASEE Annual Conference \& Exposition, Indianapolis, Indiana.

[7] Dymond, R., Lohani, V., Brogan, D., and Martinez, M. (2013) Integration of a Real-Time Water and Weather Monitoring System into a Hydrology Course, In Proc. 120th ASEE Annual Conference \& Exposition, Atlanta, Georgia.

[8] McDonald, W., Dymond, R., Lohani, V., Brogan, D., and Clark, R. (2014) Integrating a Real-Time Remote Watershed Monitoring Lab into Water Sustainability Education, In Proc. 2014 ASEE Annual Conference \& Exposition, Indianapolis, Indiana.

[9] Levesque, V., and Oberg, K. (2012) Computing Discharge Using the Index Velocity Method. 600 Techniques and Methods (U.S. Department of the Interior, U. S. G. S., Ed.), Reston, VA.

[10] Augilar, M., and Dymond, R. (2013) Innovative Technologies for Stormwater Management Programs in Small Urbanized Areas, J. Water Resour. Plann. Manage. 140.

[11] USEPA. (1986) Quality Criteria for Water, Office of Water Regulations and Standards, Washington, DC.

[12] Sanford, W., Nelms, D., Pope, J., and Selnick, D. (2012) Quantifying components of the hydrologic cycle in Virginia using chemical hydrograph separation and multiple regression analysis, U.S. Geological Survey Scientific Investigations Report. 


\section{ABOUT THE STUDENT AUTHORS}

Holly A. Clark is in her sixth year of undergraduate study at the University of Idaho. She is pursuing Bachelor of Science degrees in both Environmental Science (with a physical science emphasis) and Music History and Literature (with a clarinet emphasis). She will graduate in May 2015.

\section{PRESS SUMMARY:}

A 10 week U.S. National Science Foundation Research Experiences for Undergraduates study investigated responses of a small urban watershed, located in Blacksburg, Virginia, to acute toxicity events. High-frequency water and weather data were collected using monitoring equipment maintained by the Learning Enhanced Watershed Assessment System (LEWAS) lab, including a weather station, rain gauge, water quality sonde, and an Acoustic Doppler Current Profiler. Stormwater infrastructure fieldwork resulted in the construction of an accurate stormwater network map, which in turn allowed the identification of sources of sedimentation and other pollution from runoff events. A case study presents the response of the watershed to a winter storm event that resulted in acute chloride toxicity from runoff containing road salts.

\section{$\mathrm{AJUR}$ Volume 12 | Issue 3 | May 2015}


$\overline{A J R}$ Volume $12 \mid$ Issue $3 \mid$ May $2015 \quad 18$ 


\title{
Occurrence of Multiple Antibiotic Resistant Bacteria in Aquatic Environments in Central Minnesota
}

\author{
Megan Bollin, Dr. Ellen Jensen, and Dr. David Mitchell* \\ Department of Biology, College of Saint Benedict, St. John's University, Collegeville, MN \\ Student: mebollin@gmail.com \\ Mentors: ejensen@csbsju.edu,dmitchell@csbsju.edu*
}

\begin{abstract}
The purpose of this study was to investigate the possibility that antibiotic resistant bacteria could be isolated and identified in aquatic ecosystems in the lakes on the campus of Saint John's University and the nearby Sauk and Watab Rivers. A total of 125 isolates were collected. Seventy-nine percent of the isolates were gram negative rods. Twenty-six isolates that were resistant to seven or more antibiotics were selected for further investigation. The 26 isolates were all gram negative and members of seven different genera with Flavobacterium and Acinetobacter being the most common. Resistance coefficients were calculated based on optical density values relative to cells grown without antibiotics. Multi-drug resistant, gram negative bacteria were shown to be common in aquatic environments in central Minnesota.
\end{abstract}

\section{INTRODUCTION}

Since the beginning of widespread use of antibiotics in the 1930s and 1940s, pathogenic bacteria have been under selective pressure to evolve mechanisms to limit the effectiveness of antibiotics. As a result the minimum inhibitory concentration (MIC) for antibiotics is slowly increasing. ${ }^{1}$ MIC is defined as the lowest concentration of antibiotic that inhibits the growth of bacteria and indicates if a particular drug will be effective in treating an infection. Therefore, as time goes on, higher concentrations of antibiotics are required to kill bacteria causing infections. ${ }^{2}$ Unfortunately increased antibiotic exposure will result in even greater selective pressures for evolution to drug resistance via a variety of mechanisms. This is an important human health issue.

Initially antibiotics were only used to treat human infections. Today, antibiotics are used not only to treat infections (human, animal and some plant) but also to promote growth and prevent infections in food animals. It has been estimated that between 35 and 50 million pounds of antibiotics are used per year in the USA. ${ }^{3,4}$ Between $60 \%$ and $80 \%$ of antibiotics used in the USA are used for growth promotion and infection prevention in food animals. ${ }^{5}$ There is increasing awareness that the overuse of antibiotics (agriculturally and clinically) could lead to the evolution of "superbugs" that survive in environments where high concentrations and/or multiple classes of antibiotics are present. Previously, most research on antibiotic resistance has focused on the clinically important pathogenic bacteria causing human infections. ${ }^{6}$ However, over the past decade, it has been observed that bacteria in the environment have the potential to act as a reservoir of antibiotic resistance genes that may be transferred to pathogenic bacteria. ${ }^{7}$ Bacteria can be resistant to one or more antibiotics without being pathogenic. ${ }^{8}$ Therefore, natural environments such as lakes and streams have become targets for antibiotic resistance testing.

Unlike plants and animals - which pass their genes on only to their offspring (vertical gene transfer) - bacteria can transmit genes to other bacteria in the same environment (horizontal gene transfer). Horizontal gene transfer processes include transformation, transduction and conjugation. Since conjugation is not limited to members of the same strain or species, it is the main 
process of horizontal gene transfer in pathogenic bacteria. ${ }^{9}$ Additionally, conjugation also allows the transfer of multiple genes at once. The spread of antibiotic resistance in bacteria through horizontal gene transfer ${ }^{10}$ means that we are losing the ability to treat common bacterial infections simply and effectively. Selective pressure that develops when antibiotics are present in the environment for an extended period of time" will exacerbate this problem. The purpose of this study was to determine if bacteria that are antibiotic resistant are present in waterways surrounding St. John's University (SJU) in central Minnesota. Furthermore we wished to examine the possibility that these bacteria were resistant to multiple classes of antibiotics.

\section{METHODS}

\section{Sample Collection and Initial Plating}

Seventeen different locations were sampled including: the lakes of Saint John's University (Figure 1), the Sauk River in Cold Spring and Watab River in Saint Joseph, Minnesota (Table 1). Water samples $(100 \mathrm{~mL})$ were collected in sterile sampling bottles and placed on ice for transport to the lab. Each sample of water was plated out onto dilution broth $(\mathrm{DB}, 1$ gram tryptone per liter distilled water) agar plates. For each water sample, two spread plates were prepared. One plate was spread with $50 \mu \mathrm{L}$ of the undiluted water sample, the other with $100 \mu \mathrm{L}$ of a 1:10 dilution using dilution broth. These plates were then incubated for 48 hours at room temperature. Roughly ten colonies were selected from each sampling location for additional purification. Each colony selected was transferred using a sterile toothpick onto a new DB agar plate. Plates were incubated at room temperature for 48 hours to allow for growth. If the culture was not pure a streak plate was made on DB agar to separate the mixture. Each pure culture was then streaked onto DB agar so that colony morphology could be determined.

\section{Determination of Antibiotic Resistance}

The effect of eight antibiotics from six classes: streptomycin sulfate, penicillin G, ampicillin, tetracycline, neomycin sulfate, erythromycin, nalidixic acid, and vancomycin on the growth of the 125 isolates was assessed. These antibiotics were selected because each class acts on a slightly different target within the bacterial cell, see Table 2. Isolates were line inoculated onto DB agar plates containing $66 \mu \mathrm{g} / \mathrm{mL}$ of antibiotic using sterile toothpicks. Each plate was inoculated with four isolates, and plates were incubated at room temperature for 48 hours. The amount of growth, color, and swarming capability for each colony was recorded.

The effect of six other antibiotics on the growth of the bacterial isolates was also assessed in experiments in which the antibiotic was present in paper disks (BD/BBL). DB plates were inoculated with the bacteria to create a lawn. Then the six antibiotic disks (cephalothin, sulfamethoxazole/trimethoprim, bacitracin, nitrofurantoin, imipenem, and amoxicillin/clavulanic acid) were placed onto the plate in a circle using sterile forceps and the plates incubated for 48 hours at room temperature. The size of the zone of inhibition was then measured. If an isolate was resistance or sensitive was determined using the table packaged with the antibiotic discs.

Twenty-six isolates that were resistant to seven or more antibiotics in the previous two experiments were chosen for further investigation. In order to determine the concentration range in which these bacterial colonies are susceptible or resistant to the antibiotics, a 96 well plate assay was used. A stock solution of $0.04 \mathrm{~g}$ of antibiotic in $10 \mathrm{~mL}$ of sterile water was created. A serial twofold dilution series using TSB (tryptic soy broth, Difco/BD) was prepared for each antibiotic using the stock solution over the range of 0.25 to $64 \mu \mathrm{g} / \mathrm{mL}$. These antibiotic-TSB mixtures were then pipetted into a standard 96 well plate and inoculated with a $1 \mu \mathrm{L}$ aliquot of an overnight culture of the bacteria, one row was left uninoculated as an antibiotic-only control. The highest concentration tested $(64 \mu \mathrm{g} / \mathrm{mL})$ corresponds: $0.172 \mathrm{mM}$ penicillin $\mathrm{G}, 0.087 \mathrm{mM}$ erythromycin, $0.172 \mathrm{mM}$ 
ampicillin, $0.165 \mathrm{mM}$ amoxicillin, $0.276 \mathrm{mM}$ nalidixic acid, $0.070 \mathrm{mM}$ neomycin sulfate, $0.044 \mathrm{mM}$ streptomycin sulfate, and $0.144 \mathrm{mM}$ tetracycline. The 96 well plates were incubated for 48 hours at room temperature. The optical density $(\mathrm{OD})$ for each well was determined at $600 \mathrm{~nm}$ using a microplate reader (VERSA max, Molecular Devices) and analyzed to compute the resistance coefficient of each of these 26 organisms. Corynebacterium renale was used as a positive control since it is very susceptible to the antibiotics being tested.

Optical Density values were used to compute a numerical resistance coefficient to a particular antibiotic for each isolate. First the OD of the antibiotic-only well was subtracted from the OD of the cell + antibiotic well. This OD difference was then divided by the OD of cell $+0 \mu \mathrm{g} / \mathrm{mL}$ antibiotic well. The resulting resistance coefficient is the amount of growth suppressed by the antibiotic. A resistance coefficient of 1 means that the antibiotic did not inhibit growth. The resistance coefficients were then graphed with the resistance coefficient on the y-axis and the organism isolate on the $\mathrm{x}$-axis. The eight different bars for each organism represent the eight different antibiotic concentrations in order of increasing concentration and the corresponding resistance to that concentration.

The effect of the antibiotics penicillin $\mathrm{G}$ and streptomycin sulfate in combination was also assessed. This combination was selected based on the antibiotics mechanism of action (streptomycin inhibits protein synthesis and penicillin $G$ inhibits cell wall formation) and because the penicillin-aminoglycoside combination is used in introductory microbiology texts as an example of a combination known to have synergism. The antibiotics were tested in a 96 well assay over the range of 0 to $64 \mu \mathrm{g} / \mathrm{mL}$, with both antibiotics having the same concentration (for example, a well would have $0.5 \mathrm{ug} / \mathrm{mL}$ of penicillin $\mathrm{G}$ and $0.5 \mathrm{ug} / \mathrm{mL}$ streptomycin). Resistance coefficients were calculated as in the drug assay.

\section{IDENTIFYING SAMPLES}

The 26 bacteria used in the studies described above were identified using standard microbiological tests. Cellular morphology determined after Gram staining was used to determine which biochemical tests were used in identification. Biochemical tests included: glucose fermentation and nitrate reduction broths, Levine Eosin Methylene Blue (EMB) and Trypticase Soy Agar (TSA) plates, Triple Sugar Iron (TSI) slant, Citrate slant, gelatin deep, and Sulfide Indole Motility (SIM) deep. All test media were incubated at $37^{\circ} \mathrm{C}$ since the bacteria had been shown to grow well at human body temperature. The glucose fermentation was checked after 20 hours and the nitrate reduction was checked after 72 hours. The TSI slant was incubated for 20 hours. SIM deep and gelatin deep were both incubated at $37^{\circ} \mathrm{C}$ for 72 hours. EMB plates and TSA plates were both streaked for isolation and incubated at $37^{\circ} \mathrm{C}$ for 20 hours. ${ }^{12}$ 


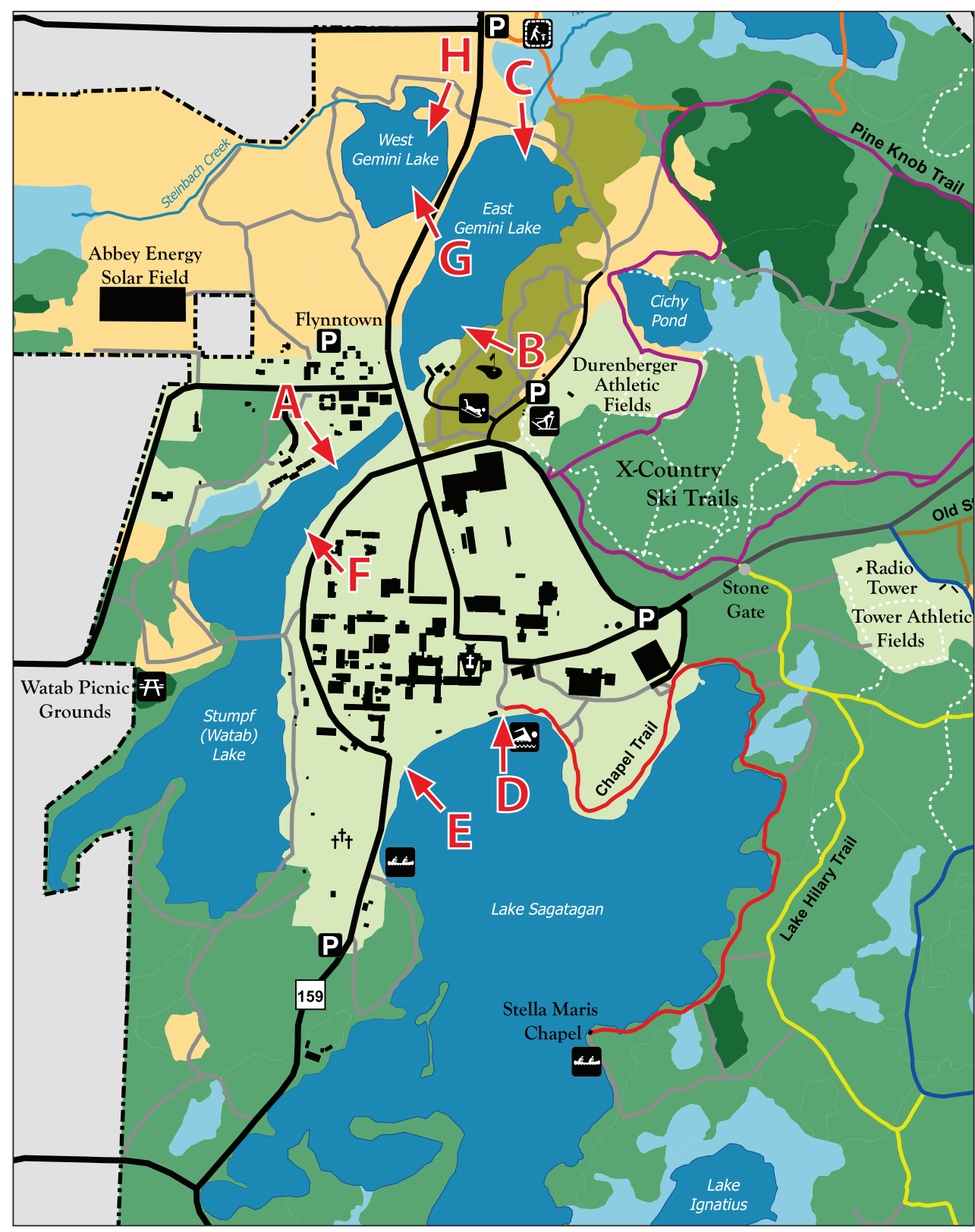

Figure 1. Sampling sites on the St. John's University (SJU) campus. 


\begin{tabular}{|c|c|c|c|c|}
\hline Location & Characteristics & $\begin{array}{l}\text { Sample } \\
\text { Letter }\end{array}$ & Coordinates & Features \\
\hline \multirow{2}{*}{ Stumpf Lake } & \multirow{2}{*}{$\begin{array}{l}\text { Man-made lake created by a } \\
\text { dam in the North Fork of the } \\
\text { Watab River }\end{array}$} & A & $\begin{array}{c}45^{\circ} 35^{\prime} 7^{\prime \prime} \mathrm{N} \\
94^{\circ} 23^{\prime} 43^{\prime} \mathrm{W}\end{array}$ & $\begin{array}{l}\text { Campus Residences } \\
\text { Nearby }\end{array}$ \\
\hline & & $\mathrm{F}$ & $\begin{array}{l}45^{\circ} 34^{\prime} 39^{\prime \prime} \mathrm{N} \\
94^{\circ} 23^{\prime} 40^{\prime \prime} \mathrm{W}\end{array}$ & $\begin{array}{l}\text { Campus Residences } \\
\text { Nearby }\end{array}$ \\
\hline \multirow{2}{*}{ East Gemini Lake } & \multirow{2}{*}{$\begin{array}{l}\text { Man-made lake formed by a } \\
\text { dam in the N. Fork Watab River. } \\
\text { Downstream from Stumpf Lake. }\end{array}$} & B & $\begin{array}{l}45^{\circ} 35^{\prime} 13^{\prime \prime} \mathrm{N} \\
94^{\circ} 23^{\prime} 35^{\prime \prime} \mathrm{W}\end{array}$ & $\begin{array}{l}\text { Where effluent from } \\
\text { SJU Waste Water } \\
\text { Treatment plant enters }\end{array}$ \\
\hline & & $\mathrm{C}$ & $\begin{array}{l}45^{\circ} 35^{\prime} 26^{\prime \prime} \mathrm{N} \\
94^{\circ} 23^{\prime} 21^{\prime \prime} \mathrm{W}\end{array}$ & $\begin{array}{l}\text { Where water flows into } \\
\text { the wetland }\end{array}$ \\
\hline \multirow{2}{*}{ West Gemini Lake } & \multirow{2}{*}{$\begin{array}{l}\text { County Road } 159 \text { separates East } \\
\text { from West Gemini, connected } \\
\text { to East Gemini by } 30 \text { inch } \\
\text { pipe. Water flows from W. to E. } \\
\text { Gemini. }\end{array}$} & G & $\begin{array}{l}45^{\circ} 35^{\prime} 24^{\prime \prime} \mathrm{N} \\
94^{\circ} 23^{\prime} 37^{\prime \prime} \mathrm{W}\end{array}$ & $\begin{array}{l}\text { East side adjacent to } \\
\text { County Road } 159\end{array}$ \\
\hline & & $\mathrm{H}$ & $\begin{array}{l}45^{\circ} 35^{\prime} 24^{\prime \prime} \mathrm{N} \\
94^{\circ} 23^{\prime} 37^{\prime \prime} \mathrm{W}\end{array}$ & $\begin{array}{l}\text { North side adjacent to } \\
\text { farm field }\end{array}$ \\
\hline \multirow{2}{*}{ Lake Sagatagan } & \multirow{2}{*}{$\begin{array}{l}\text { Natural and largest lake on } \\
\text { campus. Surrounded primarily } \\
\text { by forest }\end{array}$} & $\mathrm{D}$ & $\begin{array}{l}45^{\circ} 34^{\prime} 49^{\prime \prime} \mathrm{N} \\
94^{\circ} 23^{\prime} 29^{\prime \prime} \mathrm{W}\end{array}$ & Swimming beach \\
\hline & & $\mathrm{E}$ & $\begin{array}{l}45^{\circ} 34^{\prime} 39^{\prime \prime} \mathrm{N} \\
94^{\circ} 23^{\prime} 40^{\prime \prime} \mathrm{W}\end{array}$ & $\begin{array}{l}\text { Next to monastic } \\
\text { garden }\end{array}$ \\
\hline \multirow{5}{*}{ Sauk River } & \multirow{5}{*}{$\begin{array}{l}\text { Land use within the watershed } \\
\text { is mainly agricultural (small } \\
\text { dairy farms, hay, corn soybeans, } \\
\text { small grains) and mixed decid- } \\
\text { uous forest. The Sauk enters the } \\
\text { Mississippi on the north edge of } \\
\text { St. Cloud. }\end{array}$} & I & $\begin{array}{l}45^{\circ} 27^{\prime} 20^{\prime \prime} \mathrm{N} \\
94^{\circ} 25^{\prime} 22^{\prime \prime} \mathrm{W}\end{array}$ & $\begin{array}{l}\text { Downtown park in } \\
\text { Cold Spring }\end{array}$ \\
\hline & & $\mathrm{J}$ & $\begin{array}{l}45^{\circ} 26^{\prime} 55^{\prime \prime} \mathrm{N} \\
94^{\circ} 25^{\prime} 44^{\prime \prime} \mathrm{W}\end{array}$ & $\begin{array}{l}\text { Fishing Bridge in Cold } \\
\text { Spring }\end{array}$ \\
\hline & & K & $\begin{array}{l}45^{\circ} 27^{\prime} 22^{\prime \prime} \mathrm{N} \\
94^{\circ} 25^{\prime} 21^{\prime \prime} \mathrm{W}\end{array}$ & $\begin{array}{l}\text { Upstream of Chicken } \\
\text { Processing Plant }\end{array}$ \\
\hline & & $\mathrm{L}$ & $\begin{array}{l}45^{\circ} 27^{\prime} 48^{\prime \prime} \mathrm{N} \\
94^{\circ} 24^{\prime} 2^{\prime \prime} \mathrm{W}\end{array}$ & $\begin{array}{l}\text { Downstream from } \\
\text { Processing Plant }\end{array}$ \\
\hline & & M & $\begin{array}{l}45^{\circ} 27^{\prime} 47^{\prime \prime} \mathrm{N} \\
94^{\circ} 24^{\prime} 3^{\prime \prime} \mathrm{W}\end{array}$ & $\begin{array}{l}\text { Downstream from } \\
\text { Processing Plant }\end{array}$ \\
\hline \multirow{4}{*}{ Watab River } & \multirow{4}{*}{$\begin{array}{l}\text { Land use within the watershed } \\
\text { is mainly agricultural (small } \\
\text { dairy farms, hay, corn soybeans, } \\
\text { small grains) and mixed decid- } \\
\text { uous forest. The Watab enters } \\
\text { the Mississippi in Sartell }\end{array}$} & $\mathrm{N}$ & $\begin{array}{l}45^{\circ} 34^{\prime} 10^{\prime \prime} \mathrm{N} \\
94019^{\prime} 42^{\prime \prime} \mathrm{W}\end{array}$ & \multirow{4}{*}{$\begin{array}{l}\text { Millstream Park St. } \\
\text { Joseph. Large park } \\
\text { located at north edge } \\
\text { of the city containing } \\
\text { ball fields, Frisbee } \\
\text { golf course and picnic } \\
\text { grounds }\end{array}$} \\
\hline & & $\mathrm{O}$ & $\begin{array}{l}45^{\circ} 34^{\prime} 11^{\prime \prime} \mathrm{N} \\
94^{\circ} 19^{\prime} 40^{\prime \prime} \mathrm{W} \\
\end{array}$ & \\
\hline & & $\mathrm{P}$ & $\begin{array}{l}45^{\circ} 34^{\prime} 15^{\prime \prime} \mathrm{N} \\
94^{\circ} 19^{\prime} 41^{\prime \prime} \mathrm{W}\end{array}$ & \\
\hline & & Q & $\begin{array}{c}45^{\circ} 34^{\prime} 8^{\prime \prime} \mathrm{N} \\
94^{\circ} 19^{\prime} 44^{\prime \prime} \mathrm{W}\end{array}$ & \\
\hline
\end{tabular}

Table 1. Sampling locations including geographic coordinates and characteristics of the location. 


\begin{tabular}{|c|c|c|c|}
\hline Antibiotic Class & Target & Mode of Action & Effective Against \\
\hline $\begin{array}{c}\text { Aminoglycosides } \\
\text { (Neomycin, Streptomycin) }\end{array}$ & \multirow{3}{*}{ Protein Synthesis } & $\begin{array}{l}\text { Bind irreversibly to } 30 \mathrm{~s} \\
\text { ribosomal subunit }\end{array}$ & $\begin{array}{l}\text { Aerobic and facultative } \\
\text { gram negative and gram } \\
\text { positive bacteria }\end{array}$ \\
\hline Tetracycline & & $\begin{array}{l}\text { Bind reversibly to } 30 \mathrm{~s} \\
\text { ribosomal subunit } \\
\text { blocking binding of } \\
\text { aminoacyl-tRNA }\end{array}$ & $\begin{array}{l}\text { Gram positive and gram } \\
\text { negative bacteria }\end{array}$ \\
\hline $\begin{array}{c}\text { Macrolides } \\
\text { (Erythromycin) }\end{array}$ & & $\begin{array}{c}\text { Bind reversibly to } 23 \mathrm{~s} \\
\text { RNA of the } 50 \text { s ribosomal } \\
\text { subunit }\end{array}$ & $\begin{array}{l}\text { Gram positive and gram } \\
\text { negative bacteria }\end{array}$ \\
\hline $\begin{array}{c}\beta \text {-Lactams } \\
\text { (Penicillin G, Ampicillin) }\end{array}$ & \multirow[t]{2}{*}{ Cell Wall Synthesis } & $\begin{array}{l}\text { Irreversibly inhibit cell } \\
\text { wall synthesis by binding } \\
\text { to penicillin-binding } \\
\text { proteins preventing the } \\
\text { final crosslinking of the } \\
\text { peptidoglycan }\end{array}$ & $\begin{array}{c}\text { Gram positive, some gram } \\
\text { negative, and anaerobic } \\
\text { bacteria }\end{array}$ \\
\hline $\begin{array}{l}\text { Glycopeptides } \\
\text { (Vancomycin) }\end{array}$ & & $\begin{array}{c}\text { Inhibit cell wall synthesis } \\
\text { by preventing the addition } \\
\text { of new amino acids to the } \\
\text { peptidoglycan }\end{array}$ & Gram positive bacteria \\
\hline $\begin{array}{c}\text { Quinolone } \\
\text { (Naldixic Acid) }\end{array}$ & DNA Synthesis & Inhibits DNA gyrase & Gram negative bacteria \\
\hline
\end{tabular}

Table 2. Characteristics of the antibiotics used in the initial experiment. ${ }^{13}$

\section{RESULTS}

\section{Antibiotic Resistance}

The majority of isolates (110/125 or 88\%) were resistant to at least one antibiotic (Tables 3-5).

\begin{tabular}{|c|c|c|c|c|c|}
\hline \multirow{2}{*}{$\begin{array}{c}\text { Number of } \\
\text { drugs } \\
\text { resistant to }\end{array}$} & $\begin{array}{c}\text { Sagatagan } \\
(\mathbf{D}+\mathbf{E}) \\
\mathbf{1 5} \text { isolates }\end{array}$ & $\begin{array}{c}\text { Stumpf } \\
(\mathbf{A}+\mathbf{F}) \\
\mathbf{1 5} \text { isolates }\end{array}$ & $\begin{array}{c}\text { East Gemini } \\
(\mathbf{B}+\mathbf{C}) \\
\mathbf{1 5} \text { isolates }\end{array}$ & $\begin{array}{c}\text { West Gemini } \\
(\mathbf{G}+\mathbf{H}) \\
\mathbf{1 7} \text { isolates }\end{array}$ & $\begin{array}{c}\text { Total } \\
\mathbf{6 2} \text { isolates }\end{array}$ \\
\hline 0 & 0 & 0 & 1 & 4 & 5 \\
\hline 1 & 2 & 1 & 0 & 1 & 4 \\
\hline 2 & 5 & 1 & 1 & 4 & 11 \\
\hline 3 & 2 & 2 & 3 & 2 & 9 \\
\hline 4 & 4 & 1 & 2 & 1 & 8 \\
\hline 5 & 1 & 3 & 2 & 1 & 7 \\
\hline 6 & 0 & 4 & 2 & 1 & 10 \\
\hline 7 & 0 & 3 & 4 & 3 & 1 \\
\hline All 8 & 1 & 0 & 0 & 0 & 7 \\
\hline
\end{tabular}

Table 3. St. John's lake sample location vs. number of isolates resistant to $\mathrm{X}$ number of drugs. 


\begin{tabular}{|c|c|c|c|c|c|}
\hline \multirow{2}{*}{$\begin{array}{c}\text { Number of } \\
\text { drugs } \\
\text { resistant to }\end{array}$} & $\mathbf{I}$ & $\mathbf{J}$ & $\mathbf{K}$ & $\mathbf{L}$ & M \\
\cline { 2 - 6 } & 0 & 1 & 0 & 0 & 3 \\
\hline 0 & 2 & 1 & 1 & 0 & 1 \\
\hline 1 & 0 & 2 & 4 & 0 & 1 \\
\hline 2 & 2 & 0 & 2 & 1 & 2 \\
\hline 3 & 1 & 3 & 0 & 2 & 0 \\
\hline 4 & 1 & 0 & 0 & 1 & 0 \\
\hline 5 & 1 & 0 & 0 & 0 & 0 \\
\hline 6 & 0 & 0 & 0 & 2 & 0 \\
\hline 7 & 0 & 0 & 0 & 1 & 0 \\
\hline All 8 & & & & & \\
\hline
\end{tabular}

Table 4. Sauk River sampling site vs. number of isolates resistant to X number of drugs.

\begin{tabular}{|c|c|c|c|c|}
\hline \multirow{2}{*}{$\begin{array}{c}\text { Number of } \\
\text { drugs } \\
\text { resistant to }\end{array}$} & $\mathbf{N}$ & $\mathbf{O}$ & $\mathbf{P}$ & $\mathbf{Q}$ \\
\cline { 2 - 5 } & 1 & 1 & 2 & 2 \\
\hline 0 & 2 & 0 & 1 & 1 \\
\hline 1 & 1 & 1 & 1 & 4 \\
\hline 2 & 1 & 2 & 3 & 0 \\
\hline 3 & 1 & 2 & 0 & 0 \\
\hline 4 & 0 & 1 & 0 & 0 \\
\hline 5 & 0 & 0 & 0 & 0 \\
\hline 6 & 1 & 0 & 0 & 0 \\
\hline 7 & 0 & 0 & 0 & 0 \\
\hline All 8 & & & 0 & \multicolumn{2}{|c|}{ Watab River } \\
\hline
\end{tabular}

Table 5. Watab River sampling site vs. number of isolates resistant to X number of drugs.

The Fisher Exact Test ${ }^{14}$ was used to determine if the differences in the number of isolates resistant to $\mathrm{X}$ drugs between sites was significant. The $\mathrm{P}$ values were as follows: comparison of just the four SJU lakes $p=0.20$; comparison of five Sauk River sites $p=0.020$; comparison of four Watab River sites $\mathrm{p}=0.37$. The Fisher Exact Test was also used to compare the regions SJU lakes, Sauk River and Watab River, $\mathrm{p}=0.022$.

A data analysis using the Fisher Exact Test of the 125 isolates was also made to see if there were differences in the pattern of resistance to the different drug targets and classes for each water source (Tables 7-9). The St. John's Lakes, Sauk River and Watab River were also compared to each other using the Fisher Exact Test (Table 10). 


\begin{tabular}{|c|c|c|c|}
\hline \multirow{2}{*}{$\begin{array}{c}\text { Number of drugs } \\
\text { isolate was resistant }\end{array}$} & $\begin{array}{c}|c| \\
\text { SJU Lakes } \\
\text { (62 isolates) }\end{array}$ & $\begin{array}{c}\text { Sauk River } \\
\text { (35 isolates) }\end{array}$ & $\begin{array}{c}\text { Watab River } \\
\text { (28 isolates) }\end{array}$ \\
\cline { 2 - 4 } & 5 & 4 & 6 \\
\hline 0 & 4 & 5 & 4 \\
\hline 1 & 11 & 7 & 7 \\
\hline 2 & 9 & 7 & 6 \\
\hline 3 & 8 & 6 & 3 \\
\hline 4 & 7 & 2 & 1 \\
\hline 5 & 7 & 1 & 0 \\
\hline 6 & 10 & 2 & 1 \\
\hline 7 & 1 & 1 & 0 \\
\hline All 8 & & & \\
\hline
\end{tabular}

Table 6. Comparison of the number of isolates resistant to $\mathrm{X}$ number of drugs between SJU Lakes, Sauk River, and Watab River.

\begin{tabular}{|c|c|c|c|c|c|c|c|}
\hline \multirow[b]{2}{*}{$\begin{array}{l}\text { Antibiotic } \\
\text { Class }\end{array}$} & \multirow[b]{2}{*}{$\begin{array}{c}\text { Antibiotic } \\
\text { Target }\end{array}$} & \multicolumn{4}{|c|}{ Lake sampled } & \multirow[b]{2}{*}{$\begin{array}{l}\text { Fisher } \\
\text { p value } \\
\text { for } \\
\text { Target }\end{array}$} & \multirow[b]{2}{*}{$\begin{array}{l}\text { Fisher } \\
\text { p value } \\
\text { for } \\
\text { Class }\end{array}$} \\
\hline & & $\begin{array}{l}\text { Sagatagan } \\
(\mathrm{D}+\mathrm{E}) \\
15 \text { isolates }\end{array}$ & $\begin{array}{c}\text { Stumpf } \\
(\mathrm{A}+\mathrm{F}) \\
15 \text { isolates }\end{array}$ & $\begin{array}{c}\text { East } \\
\text { Gemini } \\
(B+C) \\
15 \text { isolates }\end{array}$ & $\begin{array}{c}\text { West } \\
\text { Gemini } \\
(\mathrm{G}+\mathrm{H}) \\
17 \text { isolates }\end{array}$ & & \\
\hline $\begin{array}{l}\text { Aminoglycosides } \\
\text { (Neomycin, } \\
\text { Streptomycin) }\end{array}$ & \multirow{3}{*}{$\begin{array}{l}\text { Protein } \\
\text { Synthesis }\end{array}$} & 15 & 15 & 13 & 13 & \multirow{3}{*}{0.057} & 0.057 \\
\hline Tetracycline & & 1 & 0 & 1 & 0 & & 0.596 \\
\hline $\begin{array}{c}\text { Macrolides } \\
\text { (Erythromycin) }\end{array}$ & & 5 & 9 & 9 & 6 & & 0.2515 \\
\hline $\begin{array}{l}\beta \text {-Lactams } \\
\text { (Penicillin G, } \\
\text { Ampicillin) }\end{array}$ & \multirow{3}{*}{$\begin{array}{l}\text { Cell Wall } \\
\text { Synthesis }\end{array}$} & 5 & 11 & 10 & 6 & \multirow{3}{*}{0.089} & 0.052 \\
\hline Glycopeptides & & & & & & & \multirow{2}{*}{0.197} \\
\hline (Vancomycin) & & 11 & 12 & 13 & 9 & & \\
\hline $\begin{array}{c}\text { Quinolone } \\
\text { (Naldixic Acid) }\end{array}$ & $\begin{array}{c}\text { DNA } \\
\text { Synthesis }\end{array}$ & 2 & 5 & 5 & 5 & 0.572 & 0.572 \\
\hline
\end{tabular}

Table 7. SJU Lake vs. number of isolates with resistance based on drug target and drug class. 


\begin{tabular}{|c|c|c|c|c|c|c|c|c|}
\hline \multirow[b]{2}{*}{$\begin{array}{l}\text { Antibiotic } \\
\text { Class }\end{array}$} & \multirow[b]{2}{*}{$\begin{array}{l}\text { Antibiotic } \\
\text { Target }\end{array}$} & \multicolumn{5}{|c|}{ Sauk River Sample } & \multirow{2}{*}{$\begin{array}{l}\text { Fisher } \\
\text { p value } \\
\text { for } \\
\text { Target }\end{array}$} & \multirow{2}{*}{$\begin{array}{l}\text { Fisher } \\
\text { p value } \\
\text { for } \\
\text { Class }\end{array}$} \\
\hline & & $\begin{array}{c}\text { I } \\
7 \text { isolates }\end{array}$ & $\begin{array}{c}\mathrm{J} \\
7 \text { isolates }\end{array}$ & $\begin{array}{c}\mathrm{K} \\
7 \text { isolates }\end{array}$ & $\begin{array}{c}\mathrm{L} \\
7 \text { isolates }\end{array}$ & $\begin{array}{c}\mathrm{M} \\
7 \text { isolates }\end{array}$ & & \\
\hline $\begin{array}{l}\text { Aminoglycosides } \\
\text { (Neomycin, } \\
\text { Streptomycin) }\end{array}$ & \multirow{3}{*}{$\begin{array}{l}\text { Protein } \\
\text { Synthesis }\end{array}$} & 4 & 5 & 4 & 4 & 1 & \multirow{3}{*}{0.118} & 0.0018 \\
\hline Tetracycline & & 0 & 0 & 0 & 1 & 0 & & 1.00 \\
\hline $\begin{array}{c}\text { Macrolides } \\
\text { (Erythromycin) }\end{array}$ & & 2 & 0 & 0 & 5 & 0 & & 0.0014 \\
\hline $\begin{array}{c}\beta \text {-Lactams } \\
\text { (Penicillin G, } \\
\text { Ampicillin) }\end{array}$ & \multirow{2}{*}{$\begin{array}{l}\text { Cell Wall } \\
\text { Synthesis }\end{array}$} & 7 & 4 & 3 & 7 & 3 & 0.056 & 0.018 \\
\hline $\begin{array}{l}\text { Glycopeptides } \\
\text { (Vancomycin) }\end{array}$ & & 2 & 3 & 3 & 6 & 3 & & 0.329 \\
\hline $\begin{array}{c}\text { Quinolone } \\
\text { (Naldixic Acid) }\end{array}$ & $\begin{array}{c}\text { DNA } \\
\text { Synthesis }\end{array}$ & 1 & 0 & 0 & 4 & 0 & 0.015 & 0.015 \\
\hline
\end{tabular}

Table 8. Sauk River samples vs. number of isolates with resistance based on drug target and drug class.

\begin{tabular}{|c|c|c|c|c|c|c|c|}
\hline \multirow{2}{*}{$\begin{array}{l}\text { Antibiotic } \\
\text { Class }\end{array}$} & \multirow{2}{*}{$\begin{array}{c}\text { Antibiotic } \\
\text { Target }\end{array}$} & \multicolumn{4}{|c|}{ Watab River Sample } & \multirow{2}{*}{$\begin{array}{l}\text { Fisher } \\
\text { p value for } \\
\text { Target }\end{array}$} & \multirow{2}{*}{$\begin{array}{l}\text { Fisher } \\
\text { p value for } \\
\text { Class }\end{array}$} \\
\hline & & $\begin{array}{c}\mathrm{N} \\
7 \text { isolates }\end{array}$ & $\begin{array}{c}0 \\
7 \text { isolates } \\
\end{array}$ & $\begin{array}{c}P \\
7 \text { isolates }\end{array}$ & $\begin{array}{c}Q Q \\
\text { 7 isolates }\end{array}$ & & \\
\hline $\begin{array}{l}\text { Aminoglycosides } \\
\text { (Neomycin, } \\
\text { Streptomycin) }\end{array}$ & \multirow{3}{*}{$\begin{array}{l}\text { Protein } \\
\text { Synthesis }\end{array}$} & 5 & 4 & 3 & 2 & \multirow{3}{*}{0.60} & 0.596 \\
\hline Tetracycline & & 1 & 0 & 0 & 0 & & 1.00 \\
\hline $\begin{array}{c}\text { Macrolides } \\
\text { (Erythromycin) }\end{array}$ & & 1 & 2 & 0 & 0 & & 0.58 \\
\hline $\begin{array}{c}\text { } \beta \text {-Lactams } \\
\text { (Penicillin G, } \\
\text { Ampicillin) }\end{array}$ & \multirow{2}{*}{$\begin{array}{l}\text { Cell Wall } \\
\text { Synthesis }\end{array}$} & 4 & 5 & 0 & 3 & \multirow{2}{*}{0.81} & 0.044 \\
\hline $\begin{array}{l}\text { Glycopeptides } \\
\text { (Vancomycin) }\end{array}$ & & 2 & 4 & 5 & 1 & & 0.17 \\
\hline $\begin{array}{c}\text { Quinolone } \\
\text { (Naldixic Acid) }\end{array}$ & $\begin{array}{c}\text { DNA } \\
\text { Synthesis }\end{array}$ & 0 & 0 & 0 & 0 & 1.00 & 1.00 \\
\hline
\end{tabular}

Table 9. Watab River samples vs. number of isolates with resistance based on drug target and drug class. 


\begin{tabular}{|c|c|c|c|c|c|c|}
\hline \multirow[b]{2}{*}{$\begin{array}{l}\text { Antibiotic } \\
\text { Class }\end{array}$} & \multirow[b]{2}{*}{$\begin{array}{c}\text { Antibiotic } \\
\text { Target }\end{array}$} & \multicolumn{3}{|c|}{ Lake Sampled } & \multirow{2}{*}{$\begin{array}{l}\text { Fisher } \\
\text { p value for } \\
\text { Target }\end{array}$} & \multirow{2}{*}{$\begin{array}{c}\text { Fisher } \\
\text { p value for } \\
\text { Class }\end{array}$} \\
\hline & & $\begin{array}{l}\text { SJU Lakes } \\
62 \text { isolates }\end{array}$ & $\begin{array}{l}\text { Sauk river } \\
35 \text { isolates }\end{array}$ & $\begin{array}{c}\text { Watab River } \\
28 \text { isolates }\end{array}$ & & \\
\hline $\begin{array}{l}\text { Aminoglycoside } \\
\text { (Neomycin, } \\
\text { Streptomycin) }\end{array}$ & \multirow{3}{*}{$\begin{array}{l}\text { Protein } \\
\text { Synthesis }\end{array}$} & 56 & 17 & 14 & \multirow{3}{*}{$<0.001$} & $<0.001$ \\
\hline Tetracycline & & 2 & 1 & 1 & & 1.00 \\
\hline $\begin{array}{c}\text { Macrolides } \\
\text { (Erythromycin) }\end{array}$ & & 29 & 7 & 3 & & $<0.001$ \\
\hline $\begin{array}{l}\beta \text {-Lactams } \\
\text { (Penicillin G, } \\
\text { Ampicillin) }\end{array}$ & \multirow{2}{*}{$\begin{array}{l}\text { Cell Wall } \\
\text { Synthesis }\end{array}$} & 32 & 24 & 12 & \multirow[t]{2}{*}{0.37} & 0.16 \\
\hline $\begin{array}{l}\text { Glycopeptides } \\
\text { (Vancomycin) }\end{array}$ & & 45 & 17 & 12 & & 0.009 \\
\hline $\begin{array}{c}\text { Quinolone } \\
\text { (Naldixic Acid) }\end{array}$ & $\begin{array}{c}\text { DNA } \\
\text { Synthesis }\end{array}$ & 17 & 5 & 0 & .002 & 0.002 \\
\hline
\end{tabular}

Table 10. Comparison between the three regions (St. John's Lakes, Sauk River and Watab River) vs. number of isolates with resistance based on drug target and drug class.

In the antibiotic disc assay $26 \%$ of the isolates were sensitive to all of the drugs (cephalothin, sulfamethoxazole/trimethoprim, bacitracin, nitrofurantoin, imipenem, and amoxicillin/clavulanic acid) tested, and 74\% were resistant to at least one of the 6 drugs. None of the isolates were resistant to imipenem. Only the St. John's Lake samples $(\mathrm{p}=0.016)$ had a Fisher Exact Test $\mathrm{p}$ value that indicated a significant difference between samples for the number of drugs the isolates were resistant to. Table 11 shows the clinical dosage and resulting serum concentration of the antibiotics used in the 96 well assay. All of the drug's peak serum concentration would be within the concentration range used in the 96 well assay $(0.5-64 \mu \mathrm{g} / \mathrm{mL})$.

\begin{tabular}{llcc}
\hline \multicolumn{1}{c}{ Antibiotic } & \multicolumn{1}{c}{ Common Dosage } & $\begin{array}{c}\text { Peak Serum } \\
\text { Concentration }\end{array}$ & Concentration (mM) \\
\hline Amoxicillin & $250 \mathrm{mg}$ per 8 hours & $5 \mu \mathrm{g} / \mathrm{mL}$ & $0.014 \mathrm{mM}$ \\
\hline Ampicillin & $250-500 \mathrm{mg}$ per 6 hours & $3 \mu \mathrm{g} / \mathrm{mL}$ & $0.009 \mathrm{mM}$ \\
\hline Streptomycin Sulfate & $1 \mathrm{~g}$ a day & $25-50 \mu \mathrm{g} / \mathrm{mL}$ & $0.026 \mathrm{mM}$ \\
\hline Nalidixic Acid & 4 g per day & $20-40 \mu \mathrm{g} / \mathrm{mL}$ & $0.172 \mathrm{mM}$ \\
\hline Penicillin G & 1 million units & $14.4 \mu \mathrm{g} / \mathrm{mL}$ & $0.043 \mathrm{mM}$ \\
\hline Erythromycin & $\begin{array}{l}250 \text { or } 500 \mathrm{mg} \\
4 \text { times a day }\end{array}$ & $7.21 \mu \mathrm{g} / \mathrm{mL}$ & $0.010 \mathrm{mM}$ \\
\hline Neomycin Sulfate & 4 g a day & $6.1 \mu \mathrm{g} / \mathrm{mL}$ & $0.010 \mathrm{mM}$ \\
\hline Vancomycin & $1 \mathrm{~g}$ per 12 hours & $20 \mu \mathrm{g} / \mathrm{mL}$ & $0.014 \mathrm{mM}$ \\
Tetracycline & $\begin{array}{l}125 \text { to } 500 \mathrm{mg} \\
\text { per } 12 \text { hours }\end{array}$ & $4-5 \mu \mathrm{g} / \mathrm{mL}$ & $0.011 \mathrm{mM}$ \\
\hline
\end{tabular}

Table 11. Clinical dosage and peak serum concentration of each antibiotic and the corresponding $\mathrm{mM}$ concentration for each antibiotic used in the 96 well assay. 
The data from the 96 well antibiotic assays on the 26 isolates were analyzed based on resistance coefficients. ${ }^{8}$ Organisms with coefficients greater than 0.8 were considered resistant to the drug at the concentration being tested. ${ }^{15}$ Graphs of the resistance coefficient data for each concentration tested showed three distinct patterns: 1) the organism's resistance coefficient was between $0.8-1$ for all concentrations tested; 2) the organism was inhibited at higher antibiotic concentrations; and 3) at some concentrations the antibiotic caused increased growth and a resistance coefficient greater than 1 (Figure 2). Table 12 shows the distribution between the three patterns for the different antibiotics.

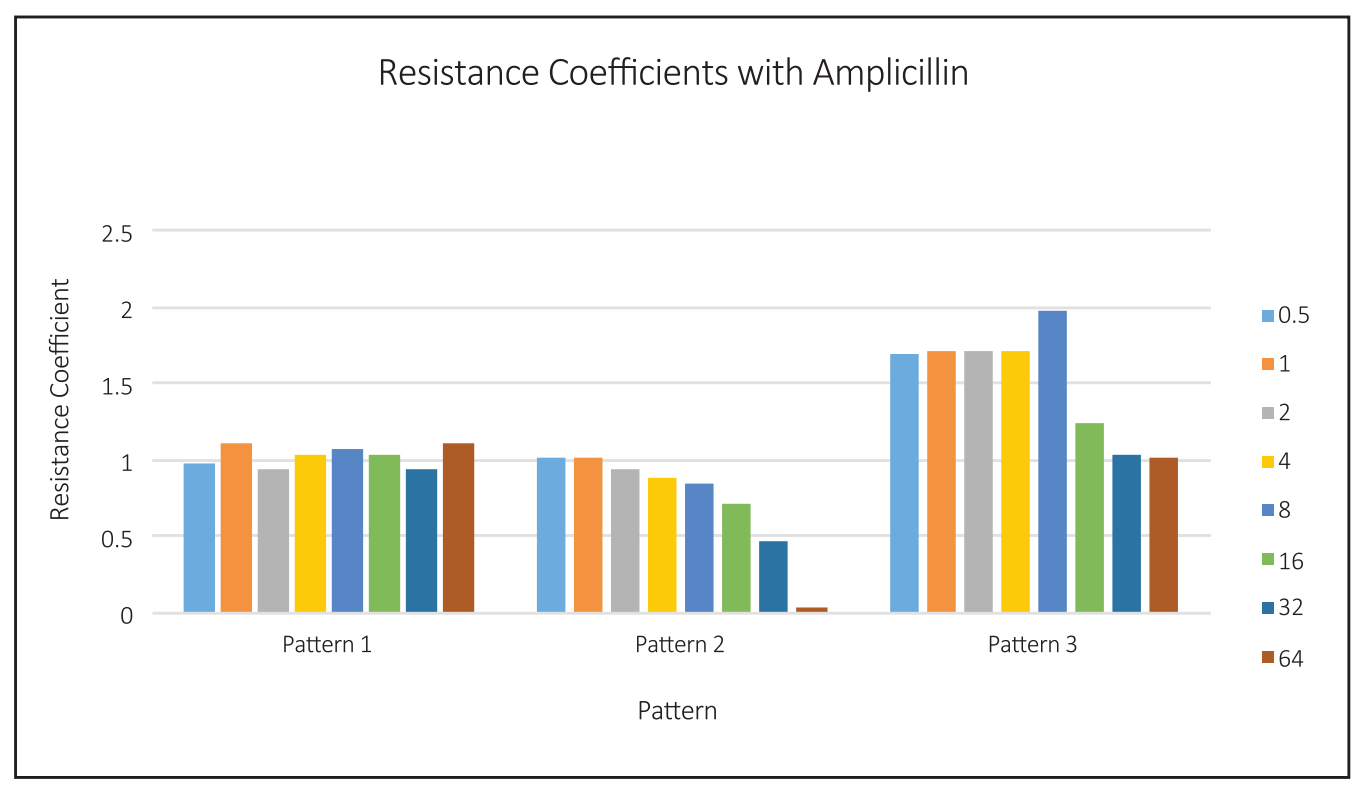

Figure 2. Resistance coefficient patterns for $0.5 \mu \mathrm{g} / \mathrm{mL}$ to $64 \mu \mathrm{g} / \mathrm{mL}$ antibiotic using ampicillin as an example.

\begin{tabular}{|c|c|c|c|c|c|c|c|c|}
\hline $\begin{array}{c}\text { Number } \\
\text { of isolates } \\
\text { with } \\
\text { pattern }\end{array}$ & 泀 & 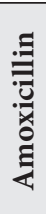 & 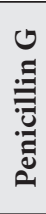 & 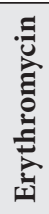 & 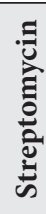 & 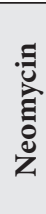 & & 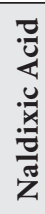 \\
\hline Pattern 1 & 17 & 18 & 19 & 19 & 17 & 15 & 0 & 19 \\
\hline Pattern 2 & 2 & 4 & 2 & 5 & 2 & 11 & 26 & 7 \\
\hline Pattern 3 & 6 & 3 & 4 & 2 & 6 & 0 & 0 & 0 \\
\hline
\end{tabular}

Table 12. Growth patterns in the 96 well plates. Pattern $1=$ Resistant at all concentrations tested,

Pattern 2 = Growth inhibited at higher concentrations, Pattern 3 = Increased growth at some concentrations

Eight of the isolates (A1, A3, A11, C6, C10, H1b, L7, N2) were resistant at all concentrations (0.5-64 $\mu \mathrm{g} / \mathrm{mL}$ ) to penicillin $\mathrm{G}$ and streptomycin in the 96 well assay. These were selected for use in a combined penicillin G plus streptomycin 96 well assay. After incubation the resistance coefficients for each well were calculated in a similar manner as in the previous experiment. All isolates showed synergism between the two drugs (Figure 3) in that higher concentrations caused an inhibition in growth. Resistance coefficients of 0.2 or less are required for successful treatment. ${ }^{15}$ Peak serum concentrations are: streptomycin 25-50 $\mu \mathrm{g} / \mathrm{mL}$ and penicillin G $14.4 \mu \mathrm{g} / \mathrm{mL}$. Two of the isolates have MICs that are low enough that the combination would be successful in treating an infection: $\mathrm{All}$ with $\mathrm{MIC}=4$ and $\mathrm{L7}$ with $\mathrm{MIC}=8$. The other six isolates would require penicillin $\mathrm{G}$ concentrations $>14.4 \mu \mathrm{g} / \mathrm{mL}$ which are not normally achieved in serum. 
Gram Stain Results of the 125 isolates

The majority of the isolates were gram negative rods (79\%) with the next most frequent morphology being gram positive endospore forming rods (8\%). The remaining isolates were subdivided between: gram positive non-endospore forming rods, cocci, gram variable rods. A Fisher Exact Test was performed in order to determine if cellular morphology was dependent on location sampled; the $\mathrm{p}$ value of 0.36 indicates the differences were not significant.

\section{Identification of the 26 Isolates:}

The two most common genera were Acinetobacter and Flavobacterium. Seven out of 26 were classified as Acinetobacter (A1, B7, C10, F3b, H3b, L6, N2) and eight as Flavobacterium (A2, A3, A6, A7, A11, C1, C6, G3). The other genera represented in these 26 isolates were Enterobacter with five isolates, Pseudomonas aeruginosa with two isolates, Proteus with one isolate, Klebsiella with one isolate, and Salmonella with one isolate. One isolate would not grow and was presumed to have died.

The Acinetobacter and Flavobacterium isolates resistance to the antibiotics that target protein and cell wall synthesis were compared (Table 13) to see if they had the same pattern. Acinetobacter isolates A1, C10 and L6 have the same pattern. Flavobacterium isolates A2, A3, and C6 have the same pattern.

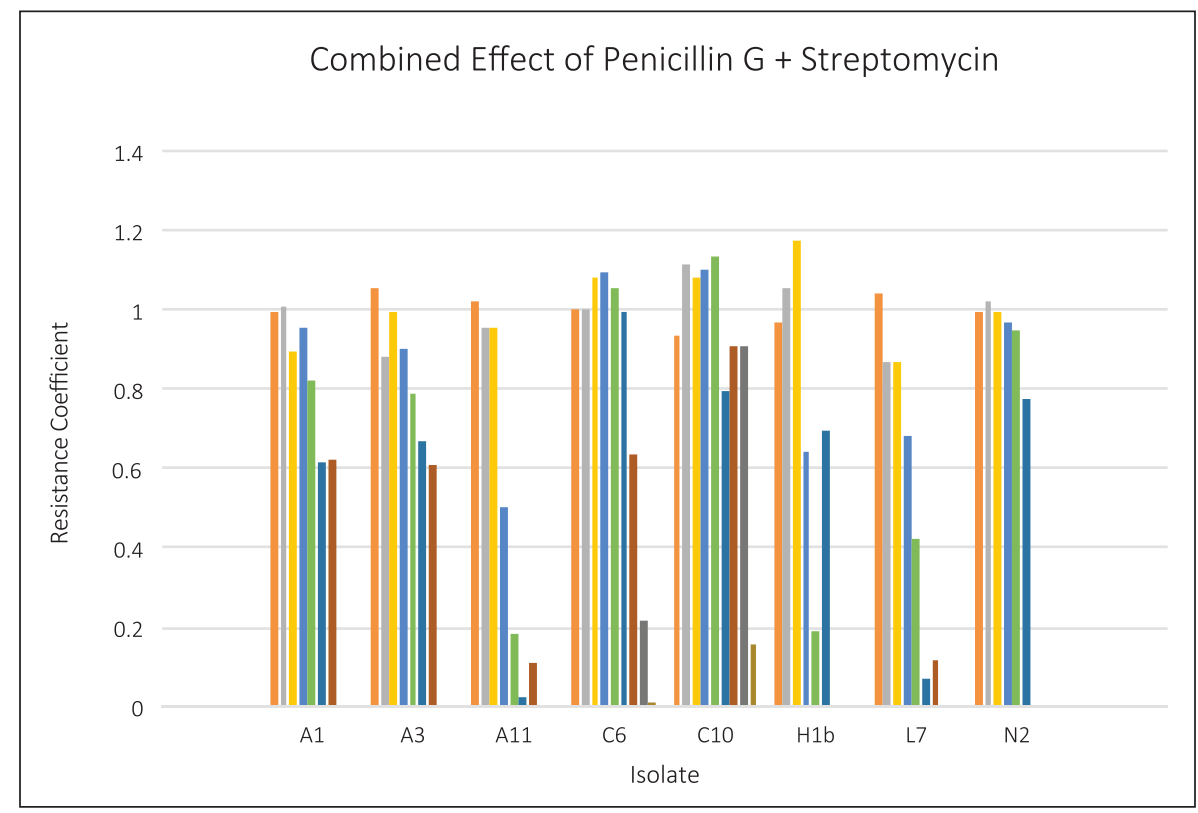

Figure 3. Combined effect of $0.25 \mathrm{ug} / \mathrm{mL}$ to $64 \mathrm{ug} / \mathrm{mL}$ penicillin $\mathrm{G}$ and streptomycin on eight isolates. 


\begin{tabular}{|l|c|c|c|c|c|c|c|c|}
\hline & \multicolumn{3}{|c|}{ Cell Wall Disruption Antibiotics } & \multicolumn{3}{c|}{ Protein Synthesis Disruption Antibiotics } \\
\hline $\begin{array}{l}\text { Acinetobacter } \\
\text { Isolates }\end{array}$ & Pen. G & Amp. & Amox. & Vanc. & Neo. & Strep. & Tet. & Eryth. \\
\hline A1 & + & + & + & + & + & + & - & + \\
\hline B7 & + & + & + & + & - & + & - & + \\
\hline C10 & + & + & + & + & + & + & - & + \\
\hline F3B & + & - & + & + & + & + & - & + \\
\hline H3B & - & - & - & + & + & - & - & - \\
\hline L6 & + & + & + & + & + & + & - & + \\
\hline N2 & + & - & + & + & + & + & - & - \\
\hline Flavobacterim & & & & & & & & \\
Isolates & + & + & + & + & + & + & - & + \\
\hline A2 & + & + & + & + & + & + & - & + \\
\hline A3 & + & + & + & + & - & - & - & + \\
\hline A6 & + & + & - & + & - & + & - & + \\
\hline A7 & - & + & + & + & - & + & - & + \\
\hline A11 & + & + & - & + & - & - & - & + \\
\hline C1 & + & - & + & + & + & + & - & + \\
\hline C6 & + & + & + & + & - & - & + \\
\hline G3 & + & + & + & + & + & + \\
\hline
\end{tabular}

Table 13. Resistance to different antibiotics for the Acinetobacter and Flavobacterium isolates. Symbols: $+=$ resistant and $-=$ susceptible

\section{DISCUSSION}

Previous studies show that the frequency of resistant bacteria in fresh water is variable and ranges from: $18.6 \%$ to $20.4 \%$ for isolates from Oregon streams and rivers to $92 \%$ for rural ground water in West Virginia. ${ }^{16-18}$ One would predict that samples collected from rural central Minnesota lakes and streams would have some antibiotic resistant bacteria present but that the frequency would be low due to the absence of a large dense population. Instead our samples had a high percentage (88\%) of bacteria resistant to at least one of eight antibiotics (Table 6). The Fisher Exact Test showed that the number of resistant isolates for the SJU lakes and Watab River samples were not significantly different (Tables 3 and 5). There was, however, a significant difference between the five Sauk River samples. Sample L which was downstream from a chicken processing plant had a higher frequency of isolates that were resistant to 6 or more drugs (Table 4). When comparing the three regions using the Fisher Exact Test $(p=0.022)$ the SJU lakes were shown to have a significantly higher number of isolates resistant to five or more drugs than the Sauk and Watab Rivers. This suggests that distinct microenvironments for horizontal gene transfer may be present in the waterways.

The antibiotic resistance data from the 125 isolates was analyzed using the Fisher Exact Test to determine if there were differences in the pattern of resistance to the different drug targets and classes either within or between the three regions (Tables 7-10). The differences seen for tetracycline resistance were not significant either within a region or between the three regions. Aminoglycoside resistance was significantly different when comparing the Sauk River samples ( $M$ had decreased number of resistant isolates), close to being significant for the SJU lake samples (decreased number of resistant isolates for West Gemini) and when the three regions were compared the SJU lakes had significantly higher number of resistant isolates than the samples from the two rivers. Differences for Erythromycin/macrolides were significant both within the Sauk River samples (increased for L) 
and between the three regions (increased for the SJU lakes). Resistance to protein synthesis inhibitors was significantly different between the regions (increased for SJU lakes). $\beta$-lactam resistance was significantly different within both the Watab River samples (decreased for sample P) and Sauk River (increased samples I and L), and almost significant within the SJU lake samples (decreased numbers for Sagatagan and West Gemini) but differences were not significant when the three regions were compared. Vancomycin/glycopeptide resistance was not significantly different when the samples within a region were compared but was significantly different when the three regions were compared to each other, in that the SJU lakes had an increased number of resistant isolates. Cell wall synthesis inhibitor differences both within and between regions were not significant. Nalidixic acid/quinolone resistance differences were only significant for the Sauk River locations and sample $\mathrm{L}$ had larger numbers of resistant isolates than the other four locations.

Two patterns of antibiotic resistance were expected to be seen in the 96 well antibiotic assays: resistant at all concentrations tested (Pattern 1) and sensitive at higher concentrations (Pattern 2). A majority of the 26 isolates acted as predicted. However, $23 \%$ of the isolates exhibited a third pattern (increased growth at some concentrations of the drug) with some drugs (ampicillin, amoxicillin, penicillin G, erythromycin, streptomycin) as shown in Figure 2. Given that antibiotics function to block the synthesis of molecules required for growth, seeing Pattern 3 seemed improbable. A search of the literature determined that Pattern 3 has been previously seen with gram negative bacteria isolated from soil especially members of the genera Pseudomonas and Burkholderia ${ }^{19}$ and Salmonella. ${ }^{20}$ This phenomenon has been named subsistence ${ }^{19,20}$ and this work provides the first evidence that this phenomenon can occur in fresh water aquatic environments. It would be interesting to see if the isolates exhibiting Pattern 3 are also able to use some antibiotics as a sole carbon source like the Salmonella isolates can. ${ }^{20}$

The most frequent bacterial cell morphology seen from all of the locations sampled was gram negative rods (79\%) followed by gram positive endospore-forming rods (8\%). The Fisher Exact Test showed the variation between locations and morphologies was not significant. Previous studies have shown that these two morphologies are the most prevalent in fresh water lakes, streams and rivers. ${ }^{16,21,24}$

In the 26 isolates chosen for identification and further study the most common genera isolated were: 31\% Flavobacterium, 27\% Acinetobacter, 19\% Enterobacter, 8\% Pseudomonas, and 4\% each for Proteus, Klebsiella, and Salmonella. Previous studies have also found these genera in fresh water but the actual percentages present varies from study to study. The percentages of Acinetobacter from our samples (27\%) are similar to those found in unpolluted lakes, streams and rivers ${ }^{21,23}$ but higher than that found in polluted streams and rivers. ${ }^{23,}{ }^{24}$ The percentages of Pseudomonas (8\%) are similar to those found for Lake Michigan and streams in Oregon ${ }^{21,24}$ but much lower than those seen in samples collected from rivers in Estonia or Oregon. ${ }^{8,16,23}$ None of the samples contained Escherichia coli and contained much lower numbers of Enterobacteriaceae than all of the other studies. Our Flavobacterium percentage (31\%) was much higher than was reported in Oregon streams, Estonia rivers and the Great Lakes ${ }^{8,24}$ but also much lower than that of polluted rivers. ${ }^{23}$

Eighty-six percent of the Acinetobacter isolates are resistant to five or more drugs and to at least one member of three or more antibiotic classes. Seventy-five percent of the Flavobacterium isolates are also resistant to five or more drugs and to at least one member of three or more antibiotic classes. Multiple Drug Resistant (MDR) is defined as being resistant to one or members of at least three classes of antibiotics. ${ }^{25}$ Pseudomonas aeruginosa, most of the other species of Pseudomonas and many other genera of gram negative bacteria have a natural resistance to penicillin $\mathrm{G}$ because the drug cannot get through their outer membrane; so observing a high frequency of resistance to this drug in isolates that are gram negative rods would be expected. Fifty-seven percent of the Acinetobacter isolates and $87.5 \%$ of the Flavobacterium isolates were resistant to ampicillin. Other studies have also shown high levels of resistance to ampicillin: 90\% of Pseudomonas isolates were resistant, $100 \%$ of Acinetobacter Abc but less than $1 \%$ of environmental Acinetobacter species were resistant, and $73 \%$ of Flavobacterium were resistant. ${ }^{23,26,27}$ If we look at the drugs that most of the 
isolates were sensitive to (tetracycline and imipenem) we see that other studies also saw no-tolow frequencies of resistance for environmental isolates of these three genera. ${ }^{23},{ }^{25}$ All three genera (Acinetobacter, Flavobacterium and Pseudomonas) are known for: their metabolic versatility especially for carbon sources utilized, ability to cope with environmental stress, large genomes and proven ability for horizontal gene transfer. ${ }^{25,26,28,29}$

Acinetobacter, Flavobacterium and Pseudomonas are genera for whom the normal habitat of most species is fresh water. The three genera also contain some species that are recognized as being opportunistic pathogens causing human infections, especially nosocomial infections. Pseudomonas aeruginosa has long been known to cause opportunist infections but the Acinetobacter $A b c$ complex has only emerged over the last 20 years as being an important cause of nosocomial infections. ${ }^{30}$ Pseudomonas aeruginosa and the Acinetobacter Abc complex are responsible for causing more than $80 \%$ of non-enteric gram negative rod infections in humans. ${ }^{31}$ It would be interesting to key out the Acinetobacter and Flavobacterium isolates to see if they are species that are strictly environmental or human associated and opportunistic pathogen like the Acinetobacter $A b c$ complex.

In conclusion this study demonstrates that antibiotic resistant bacteria can be isolated from a number of water sources on our campus and in the local community. The majority of these bacteria would be expected to be present in these waterways yet the level of resistance to antibiotics at clinical concentrations (and above) and resistance to multiple classes of antibiotics is disconcerting. In addition our data indicates that patterns of antibiotic resistance can be quite different in nearby waterways. Taken together with the works cited in this paper questions about the (over) use of antibiotics in agriculture and medicine may need to be addressed much more critically in the near future.

\section{REFERENCES}

[1] Fernandez, L., Breidenstein, E., and Hancock, R. (2012) Importance of Adaptive and Stepwise Changes in the Rise and Spread of Antimicrobial Resistance, John Wiley \& Sons, Inc., Hoboken, New Jersey.

[2] Martinez, J. L. (2008) Antibiotics and antibiotic resistance genes in natural environments, Science $321,365-367$.

[3] Davies, J., and Davies, D. (2010) Origins and evolution of antibiotic resistance, Microbiology and Molecular Biology Reviews : MMBR 74, 417-433.

[4] Shea, K. M. (2003) Antibiotic resistance: what is the impact of agricultural uses of antibiotics on children's health?, Pediatrics 112, 253-258.

[5] Silbergeld, E. K., Graham, J., and Price, L. B. (2008) Industrial food animal production, antimicrobial resistance, and human health, Annual Review of Public Health 29, 151-169.

[6] Wright, G. (2012) Antibiotic Resistome: A Framework Linking the Clinic and the Environment, In Antimicrobial Resistance in the Environment (Keen, P., and Montforts, M., Eds.), John Wiley \& Sons, Inc., Hoboken, New Jersey.

[7] Levy, S. (2012) Introduction. Antimicrobial Resistance in the Environment, In Antimicrobial Resistance in the Environment (Keen, P., and Montforts, M., Eds.), John Wiley \& Sons, Inc., Hoboken, New Jersey.

[8] Voolaid, V., Joers, A., Kisand, V., and Tenson, T. (2012) Co-occurrence of resistance to different antibiotics among aquatic bacteria, BMC Microbiology 12, 225.

[9] Roberts, M. (2012) Mechanisms of Bacterial Antibiotic Resistance and Lesions Learned from Environmental Tetracycline-Resistant Bacteria, In Antimicrobial Resistance in the Environment (Keen, P., and Montforts, M., Eds.), John Wiley \& Sons, Inc., Hoboken, New Jersey. 
[10] van Hoek, A. H., Mevius, D., Guerra, B., Mullany, P., Roberts, A. P., and Aarts, H. J. (2011) Acquired antibiotic resistance genes: an overview, Frontiers in Microbiology 2, 203.

[11] Couce, A., and Blazquez, J. (2009) Side effects of antibiotics on genetic variability, FEMS Microbiology Reviews 33, 531-538.

[12] Jensen, E. (2013) Microbiology Laboratory Manual (Biology 212), Biology Department of the College of Saint Benedict/St. John's University, Collegeville, MN.

[13] ASM. Chapter 67, In Manual of Clinical Microbiology (Murray, P., Ed.), ASM Press, Washington DC.

[14] Kirkman, T. (1996) Statistics to Use, http://www.physics.csbsju.edu/stats/ accessed July 2014

[15] Jorgensen, J., and Turnidge, J. (2003) Susceptibility Test Methods: Dilution and Disc Methods, In Manual of Clinical Microbiology (Murray, P., Ed.), ASM Press, Washington DC.

[16] Armstrong, J., Shigeno, D., Calomiris, J., and Seidler, R. (1982) Antibiotic-resistant Bacteria in Drinking Water, Applied and Environmental Microbiology 42, 277-283.

[17] Bell, J. B., Elliott, G. E., and Smith, D. W. (1983) Influence of sewage treatment and urbanization on selection of multiple resistance in fecal coliform populations, Applied and Environmental Microbiology 46, 227-232.

[18] McKeon, D., Calabrese, J., and Bissonnette, G. (1995) Antibiotic Restant Gram-negative Bacteria in Rural Groundwater Supplies, Water Research 29, 1902-1908.

[19] Barnhill, A. E., Weeks, K. E., Xiong, N., Day, T. A., and Carlson, S. A. (2010) Identification of multiresistant Salmonella isolates capable of subsisting on antibiotics, Applied and Environmental Microbiology 76, 2678-2680.

[20] Dantas, G., Sommer, M. O., Oluwasegun, R. D., and Church, G. M. (2008) Bacteria subsisting on antibiotics, Science 320, 100-103.

[21] Bennett, E. (1969) A study of the Distribution of Hetertrophic Bacteria in the Great Lakes, Bacteriology Branch of the Laboratories Division of the Ontario Water Resources Commission.

[22] Ash, R., Mauck, B., and Morgan, M. (2002) Antibiotic resistance of gram-negative bacteria in rivers, United States, Emerging Infectious Diseases 8, 713-716.

[23] Kelch, W. J., and Lee, J. S. (1978) Antibiotic resistance patterns of gram-negative bacteria isolated from environmental sources, Applied and Environmental Microbiology 36, 450-456.

[24] LeChevallier, M. W., Seidler, R. J., and Evans, T. M. (1980) Enumeration and characterization of standard plate count bacteria in chlorinated and raw water supplies, Applied and Environmental Microbiology 40, 922-930.

[25] Vaz-Moreira, I., Nunes, O. C., and Manaia, C. M. (2012) Diversity and antibiotic resistance in Pseudomonas spp. from drinking water, The Science of the Total Environment 426, 366-374.

[26] Guardabassi, L., Dalsgaard, A., and Olsen, J. E. (1999) Phenotypic characterization and antibiotic resistance of Acinetobacter spp. isolated from aquatic sources, Journal of Applied Microbiology 87, 659-667.

[27] Holmes, B., Snell, J. J., and Lapage, S. P. (1979) Flavobacterium odoratum: a species resistant to a wide range of antimicrobial agents, Journal of Clinical Pathology 32, 73-77.

[28] Projan, S. (2007) Genome Size Matters, Antimicrobial Agents and Chemotherapy 51, 1133-1134. 
[29] Touchon, M., Barbier, P., Bernardet, J. F., Loux, V., Vacherie, B., Barbe, V., Rocha, E. P., and Duchaud, E. (2011) Complete genome sequence of the fish pathogen Flavobacterium branchiophilum, Applied and Environmental Microbiology 77, 7656-7662.

[30] Peleg, A. Y., Seifert, H., and Paterson, D. L. (2008) Acinetobacter baumannii: emergence of a successful pathogen, Clinical Microbiology Reviews 21, 538-582.

[31] Sader, H. S., Streit, J. M., Fritsche, T. R., and Jones, R. N. (2004) Antimicrobial activity of daptomycin against multidrug-resistant Gram-positive strains collected worldwide, Diagnostic Microbiology and Infectious Disease 50, 201-204.

\section{ABOUT THE STUDENT AUTHOR}

Megan Bollin worked on this project in the summer of 2013 as part of her honors thesis in Biology at the College of Saint Benedict/Saint John's University. She graduated with a Bachelor of Arts degree in Biology in May 2014 and while an undergraduate spent a semester studying in London. She currently works for a biotechnology firm in the Twin Cities.

\section{PRESS SUMMARY}

The (over)use of antibiotics in agriculture and medicine over the last few decades has raised serious questions about the evolution of bacteria that have become resistant to standard antibiotics. In this paper, we sampled several local waterways in and around St. John's University in central Minnesota to determine if bacteria growing in these smaller, isolated areas had developed resistance to standard antibiotics and if so which classes of antibiotics were they now able to resist. The results indicate that bacteria growing in these areas are resistant to many common antibiotics. 
$\overline{A J R}$ Volume 12 | Issue 3 | May 2015 


\title{
Effect of a Worksite Walking Competition on Health- Related Quality of Life Among University Employees
}

\author{
Breanna Z. Orozco*, Lisa J. Leininger, and Kendra L. Contente \\ Department of Kinesiology, California State University, Monterey Bay, CA \\ Students:borozco@csumb.edu*,kcontente@csumb.edu \\ Mentor:lleininger@csumb.edu
}

\begin{abstract}
Worksite health promotion programs (WHPPs) aim to improve the health and wellness of employees in an effort to improve health related quality of life (HRQOL). The effect of exercise on improving $\mathrm{HRQOL}$ is well documented among clinical populations. However, few studies have examined the effect of WHPPs on HRQOL. The purpose of this study was to investigate the effect of a six-week "Workplace Walk-Off Competition" (WWC) on HRQOL among university employees. One hundred and nine university employees were included in this study (WWC group: $n=47$, Control group: $n=62$ ). All study participants completed the Short Form 12 Question, Version 2 (SF-12v2), a HRQOL questionnaire, before and after the WWC. The SF-12v2 questionnaire determines HRQOL based on two components and reports scores for a physical component summary (PCS) score and a mental component summary (MCS) score. A two-way repeated measures ANOVA was performed on PCS and MCS scores, followed by dependent t-tests for each group. There was no significant difference in PCS or MCS scores between the groups. Further, there were no statistically significant changes in PCS or MCS scores ( $p>$.05) among either group, following the six-week WWC. Although much research deems WHPPS effective for improving many health indicators, this short-term program was not effective in improving PCS and MCS components of HRQOL.
\end{abstract}

\section{KEYWORDS}

Quality of Life, Health Promotion, Walking Competition, University Employees

\section{INTRODUCTION}

According to the American Time Survey for 2012, Americans spend a significant amount of their day at work, averaging a total of 8.8 hours. ${ }^{1}$ The significant time spent at the workplace creates an ideal setting for structuring health behavior initiatives, such as worksite health promotion programs (WHPPs). Research demonstrates WHPPs focused on physical activity offer benefits for both the employee and the organization. Individual employee benefits include improvements in health indicators such as body mass index, perceived stress, cholesterol, blood pressure levels and physical activity amounts. ${ }^{2-6}$ Organizational benefits include decreases in absenteeism, increases in job productivity, and substantial health care savings. ${ }^{7,8}$

One of the primary goals of improving individual health indicators, including physical activity, is to improve perceived quality of life. Health-related quality of life (HRQOL) is a multifaceted concept encompassing many health related characteristics. HRQOL dimensions are frequently separated into physical and mental components. ${ }^{9,10}$ Research indicates that HRQOL can be improved with physical activity participation; higher physical activity levels are associated with an increased HRQOL."

Utilization of physical activity programs as a method to improve HRQOL in patients with various chronic diseases are well documented. ${ }^{10,12}$ However, fewer studies have focused on university physical activity programs' effect on HRQOL among employees. A recent study found that 
university employees participating in a four-month walking program increased the mental component ratings of HRQOL, but showed no significant changes in physical component ratings. ${ }^{9}$ Due to the limited and mixed results of the current literature, the purpose of this study was to determine the effect of a six-week worksite walking competition on HRQOL among university employees.

\section{METHODS}

\section{Program Description}

The Workplace Walk-Off Competition (WWC) was a six-week walking program open to all employees $(n=878)$ at a small California university. The WWC was a collaborative effort between the Kinesiology and Human Resource Departments. The program was staffed by two Human Resource administrators, one Kinesiology professor, and 10 undergraduate Kinesiology students.

A total of 170 university employees participated in the WWC (95\% of WWC participants completed all components of the program). To provide accountability and foster comradery, competition participants self-assembled into teams of five (for a total of 34 teams). Pedometers were used to quantify physical activity amounts. Each team member recorded their total number of steps per week onto a website designed and maintained by program staff. The WWC included a "Kick Off" event, three "Lunch \& Learn" meetings, and a final awards ceremony. During the "Kick Off" event researchers explained the rules of the WWC and introduced the teams. The "Lunch \& Learns" served as an educational component of the competition and were presented by the Kinesiology professor and included topics such as, "Choose This, Not That," "Healthy Shopping and Cooking for the Family," and "Healthy Living for the Busy Family." In an effort to encourage an increase of step counts, WWC facilitators set weekly step goals for WWC participants. The weekly step goal began at 30,000 steps for week one and gradually increased by 10,000 steps each subsequent week. All participants in the WWC, who met the step goal for the week, were entered into a weekly prize drawing. Weekly prizes were donated by local businesses.

\section{Participants}

One week prior to the start of the WWC, all university employees were sent an email soliciting participation in an online survey. Study procedures and information were provided to all participants of the study, and informed consent was obtained. Approval to conduct the study was granted by the University's Human Subjects Institutional Review Board. One week following the WWC, the same survey was sent via email. A total of 109 employees completed both surveys (WWC group $=62$, control group $=47$ ).

\section{Protocol}

This study was a pre/post design, including a WWC group and control group. The WWC group included individuals who participated in the six-week WWC. The control group consisted of employees who chose not to participate in the WWC.

Data were collected the week prior to the start, and the week following, the WWC. The Short Form, 12 Question, Version 2 (SF 12v2) questionnaire was used to assess HRQOL. The SF-12v2 questionnaire was administered via an online link.

The SF-12v2 questionnaire is a validated and reliable health survey including 12 -questions. ${ }^{13}$ For example, one question of the SF-12v2 reads "During the past 4 weeks, how much of the time has your physical health or emotional problems interfered with your social activities (like visiting with friends, relatives, etc.)?" The SF-12v2 questionnaire is divided into two quality of life components, the Mental Component Summary (MCS) and Physical Component Summary (PCS). Each

\section{$\mathrm{AJUR}$ Volume 12 | Issue 3 | May 2015}


component is then divided into an additional four categories. The MCS is divided into vitality, social functioning, role-emotional and mental health. The PCS is divided into physical functioning, role physical, bodily pain, and general health. The MCS and PCS are scored from $0-100$, using norm-based methods. A linear t-score transformation method was used so that both the PCS and MCS have a mean of 50 and a standard deviation of 10 in the general U.S. population (from 1998). ${ }^{13}$ Therefore, a higher score would indicate a higher HRQOL. Health Outcomes Scoring Software (Quality Metric, Lincoln, RI) was used to score the SF-12 questionnaires. To further explore perceived overall health, the authors chose to evaluate one of the questions from the SF 12v2. This question asked the respondent to indicate how they perceived their overall health, with the choices of: Excellent, Very Good, Good, Fair, or Poor.

\section{Statistical Analysis}

A two-way analysis of variance (ANOVA) with repeated measures was used to analyze the PCS and MCS scores. The independent variables for the study were time (pre and post WWC) and participation status (participation in WWC, or no participation in the WWC). Dependent variables were PCS and MCS scores. To assess within-group changes in PCS and MCS scores, t-tests for dependent samples were performed. To further explore perceived overall health, a Chi-Square for linearity was performed for perceived health ratings between groups. Data analyses were conducted using SPSS 21.0. The level of significance was set at $\alpha=.05$.

\section{RESULTS}

Demographics variables were self-reported online following completion of the SF 12v2 questionnaire $(\mathrm{n}=109)$. Demographics of the study participants are shown in Table 1.

\begin{tabular}{|c|c|c|c|c|}
\hline & \multicolumn{2}{|c|}{ Control Group $(n=47)$} & \multicolumn{2}{|c|}{ WWC Group $(n=62)$} \\
\hline & $\mathrm{N}$ & $\%$ & $\mathrm{~N}$ & $\%$ \\
\hline \multicolumn{5}{|l|}{ Gender } \\
\hline Male & 12 & 25.5 & 12 & 19.4 \\
\hline Female & 34 & 72.3 & 49 & 79 \\
\hline Decline to State & 1 & 2.1 & 1 & 1.6 \\
\hline \multicolumn{5}{|l|}{ Ethnicity } \\
\hline Hispanic/Latino & 7 & 14.9 & 7 & 11.3 \\
\hline White & 29 & 61.7 & 40 & 64.5 \\
\hline Other & 9 & 19.1 & 12 & 19.4 \\
\hline Decline to State & 2 & 4.3 & 3 & 4.8 \\
\hline \multicolumn{5}{|l|}{ Job Position } \\
\hline Staff & 26 & 55.3 & 41 & 66.1 \\
\hline Faculty & 17 & 36.2 & 12 & 19.4 \\
\hline Administration & 3 & 6.4 & 8 & 12.9 \\
\hline Decline to State & 1 & 2.1 & 1 & 1.6 \\
\hline
\end{tabular}

Table 1. Study participant demographics. 


\section{PCS Scores}

A two-way repeated measure (2x2) ANOVA was computed with time as the within-subject factor and participation as the between subject factor. There was no significant effect found for time $(\mathrm{p}=.968)$ or an interaction between time and participation $(\mathrm{p}=.542)$. This indicates that there is no difference in pre or post PCS scores between either the control or WWC group.

Results of the paired sample t-test indicated no significant difference $(\mathrm{p}=.624)$ in PCS score pre$(M=51.9 \pm 8.6)$ to post-WWC $(M=52.6 \pm 7.47)$ among the WWC group. Among the control group, there was also no significant difference $(\mathrm{p}=.704)$ in PCS score pre- $(\mathrm{M}=52.5 \pm 7.0)$ to post-WWC $(\mathrm{M}=51.9 \pm 7.4)$. Figure 1 shows mean scores for PCS between the WWC group and the control group.

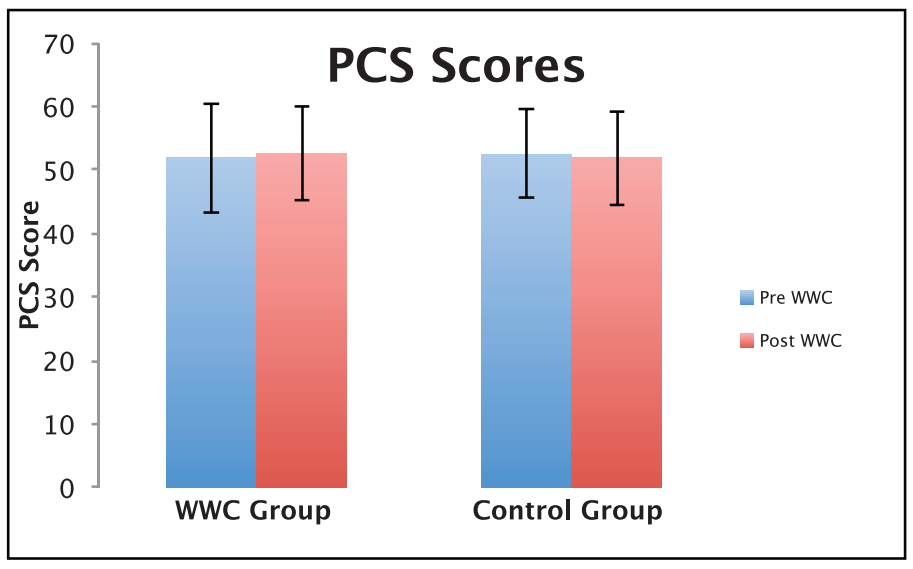

Figure 1. Mean PCS scores pre- and post-WWC between groups.

\section{MCS Scores}

A two-way repeated measure $(2 \times 2)$ ANOVA was computed with time as the within-subject factor, and participation as the between subject factor. There was no significant effect found for time $(\mathrm{p}=.784)$ or an interaction between time and participation $(\mathrm{p}=.631)$.This indicates that there is no difference in pre or post MCS scores between either the control or WWC group.

Results of the paired sample t-test indicate no significant differences $(\mathrm{p}=.885)$ in MCS score pre$(\mathrm{M}=49.4 \pm 8.5)$ to post-WWC $(\mathrm{M}=49.2 \pm 9.6)$ for the WWC group. There were also no significant differences in MCS score pre- $(M=48.8 \pm 8.6)$ to post-WWC $(M=49.7 \pm 8.5)$ for the control group. Figure 2 shows mean scores for MCS for the WWC group and the control group.

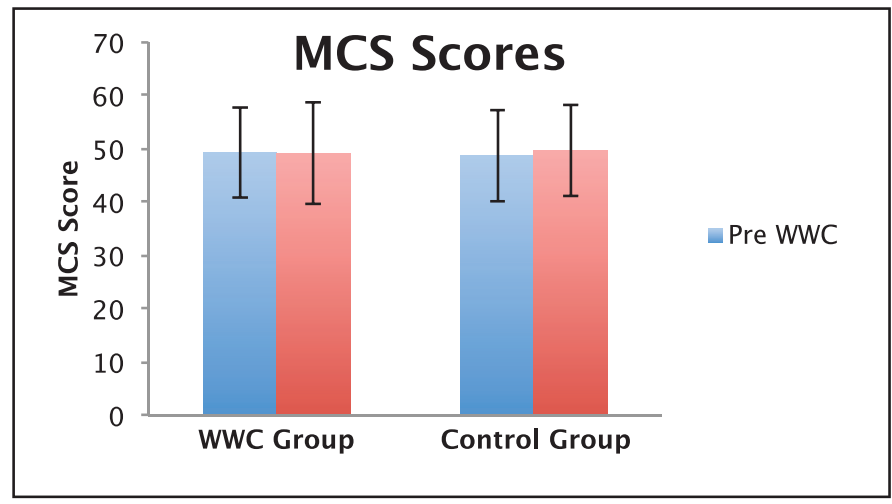

Figure 2. Mean MCS scores pre- and post-WWC between groups. 


\section{Perceived Health Ratings}

As part of the HRQOL survey, study participants were asked to rate their perceived general health on a 1-5 Likert scale (1=Excellent, $2=$ Very Good, $3=$ Good, 4=Fair, or 5=Poor. Figures 3 and 4 report percentages of respondents for each health category, pre- and post-WWC. Most respondents indicated that their health was either "very good" or "good."

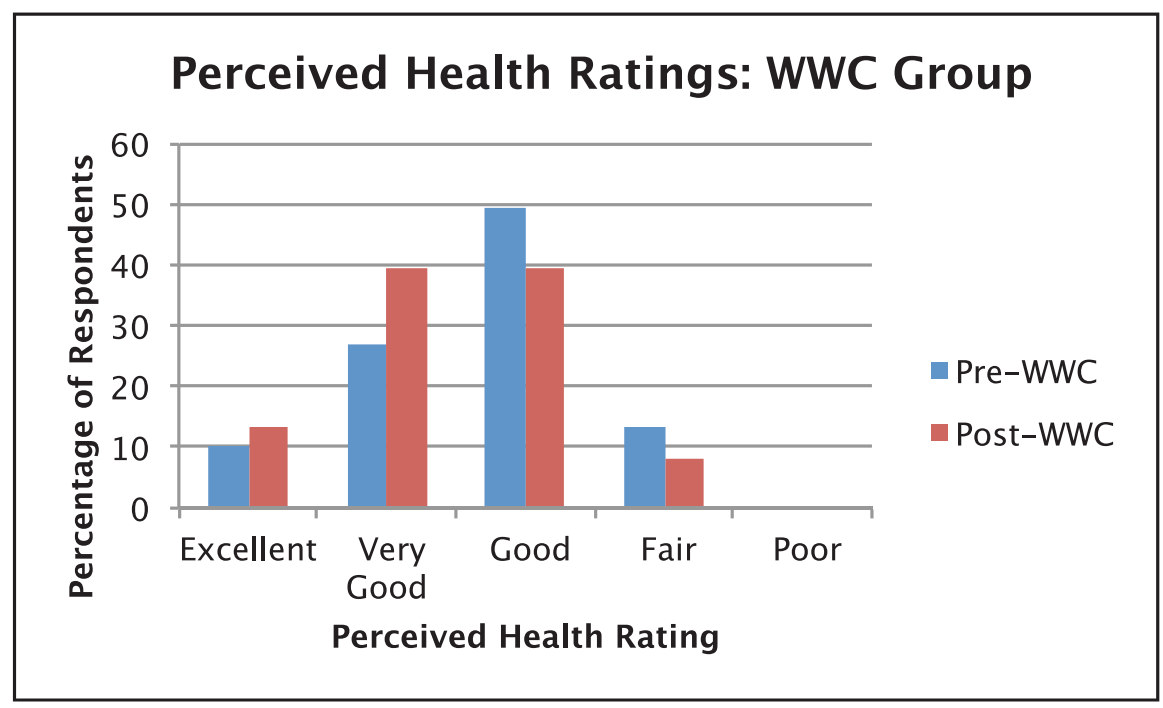

Figure 3. WWC group perceived health ratings pre- and post-WWC.

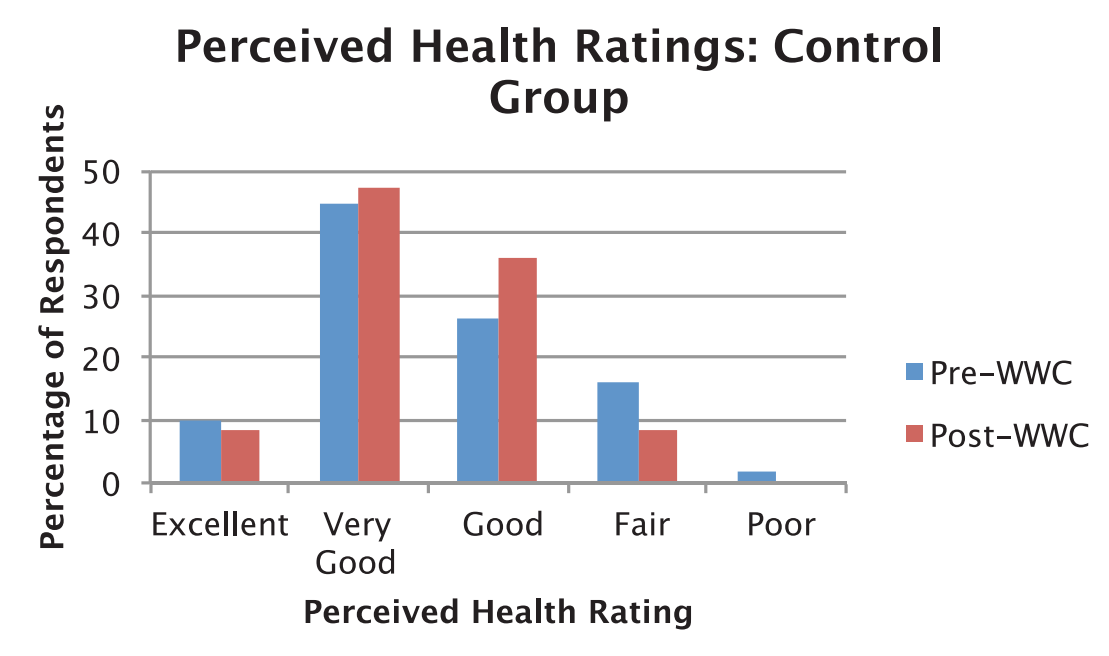

Figure 4. Control group perceived health ratings pre- and post-WWC. 
To further explore the perceived health ratings, A Chi-Square for linearity was performed to assess differences between groups. Results indicate that there were no difference in health rating pre-WWC $\left(\chi^{2}=.41, \mathrm{p}=.53\right)$ between the WWC and control groups. Further, there were no differences in health rating post-WWC $\left(\chi^{2}=.03, \mathrm{p}=.85\right)$ between the WWC and control groups.

\section{DISCUSSION}

The purpose of this study was to examine if HRQOL could improve with participation in a workplace walking competition. The results of this study indicate that the program did not have an effect on HRQOL. Although not statistically significant, there was a small increase in PCS score in the WWC group and a decrease for the control group. However, results were reversed in terms of the MCS scores. MCS scores for the WWC group decreased, while MCS scores for the control group increased. The outcome of this study is consistent with previous findings revealing no statistically significant change in PCS following a worksite health promotion program. ${ }^{9}$

The lack of improvement in HRQOL may be a result of the time of the year this study took place, even though the researchers attempted to address this through use of a control group. The study began during the middle of the academic semester and concluded during the last week of the school year. The end of the semester is a considerably busy time for staff and faculty, as they begin preparation for end of the year tasks. For instance, faculty are immersed in end of the year projects, exams, and finalizing grades. Staff members are just as busy by finishing paper work for graduation, counseling students, or completing other campus projects.

The major limitation of this study was the short time frame of the program. HRQOL is extremely complex and multifaceted. This program focused solely on physical activity participation and did not take an overall holistic approach toward improving health (e.g. nutrition, stress management, etc.) This may indicate that physical activity alone at the worksite is not sufficient to improve HRQOL. Also, the distribution of the WWC group and the control group was not randomized. This was not possible due to the voluntary nature of the WWC and survey solicitation. It may be that those who choose to participate in the study already rate their HRQOL higher in comparison to the rest of the population. Finally, as previously noted, of the $170 \mathrm{WWC}$ participants, 109 (64\%) participated in the study. Thus, the results of this study may not accurately reflect the entire participant population. Further, only 19\% of the employee population participated in the WWC. Therefore, these results may not be generalizable to the entire university population.

Most of the study participants rated their health as either "very good" or "good." It appears that the individuals included in this study have overall perceived good health. Due to this, impact of a short term walking program may be limited. Therefore, future programs and thus, research, should focus on "high-risk" individuals, or those who rate their health as "fair" or "poor." Additionally, future research projects could objectively stratify risk at the onset of programming, due to the fact there may be some difference in what individuals perceive as good health and actual health indicators.

\section{Implications and Areas of Future Research}

This study has many implications for both administrators and participants of WHPPs. Although there are several studies indicating positive health changes for employees (e.g. physical activity level, stress), these results are consistent with previous research indicating little change in HRQOL. Therefore, it may be beneficial for program administrators to weigh the cost versus potential benefits when initiating a program. Further, if a program already exists, an administrator may elect to do a cost-benefit analysis during the evaluation process. Administrators should also be selective in the claims and language they use when marketing the program, and be very precise. For instance, much research indicates that physical activity increases with WHPP participation, so an 
administrator should choose that claim rather than indicating overall HRQOL increases. Additionally, participants should be critical consumers of these programs, and also weigh the cost (whether it be financial, time, etc.) in relation the realistic benefits they may gain. For example, attending a local gym may be a more feasible health practice for their lifestyle, rather than attending programs at the worksite.

Secondly, based on results of this and previous studies, administrators of such programs should increase the duration of programs in order to potentially affect HRQOL. Harding et al. ${ }^{9}$ recently examined a similar worksite walking program. Similar to the WWC used in this study, participants used pedometers, with a 10,000 step per day goal. Harding et al.'s program also had participants report their steps with a program website, and administrators sent monthly encouraging emails, which is nearly identical to this program. Results indicated that the MCS score for HRQOL increased for participants who had participated in the program in repeated years. The major difference between their program and the current study is the duration of the walking program. Harding et al. examined the effects after four months of participation, while this study was conducted over six weeks. Therefore, based on the differences in results, the authors postulate that a longer worksite program may be necessary to illicit increases in HRQOL. If HRQOL is the main goal of the program, administrators may also wish to implement different interventions to improve HRQOL. Due to the complex nature of HRQOL, more comprehensive, longer term programs may be necessary to illicit such increases in HRQOL.

Areas of future research should focus on which components of a WHPP are most effective for improving health indicators. For example, this program had many components: the walking competition, use of teams, use of pedometers, website information, lunch-and-learn presentations, and incentives. All of these components may or may not contribute to the success of similar programs, and thus should be evaluated. Programs of longer duration should also be studied, with perhaps different measurement tools for HRQOL.

In conclusion, our findings indicate that a six-week worksite walking competition had limited impact on HRQOL. However, ample research indicates WHPPs are beneficial for both the individual and organization. Overall, more comprehensive, long-term programs at universities should be studied in an effort to help improve health and quality of life among employees.

\section{REFERENCES}

[1] (2012) American Time Use Survey, In Bureau of Labor Statistics, United States Department of Labor, http://www.bls.gov/tus/datafiles_2012.htm; cited 2014 June 25

[2] Haines, D. J., Davis, L., Rancour, P., Robinson, M., Neel-Wilson, T., and Wagner, S. (2007) A pilot intervention to promote walking and wellness and to improve the health of college faculty and staff, Journal of American College Health: J of ACH 55, 219-225.

[3] Gold, B. C., Burke, S., Pintauro, S., Buzzell, P., and Harvey-Berino, J. (2007) Weight loss on the web: A pilot study comparing a structured behavioral intervention to a commercial program, Obesity $15,155-164$.

[4] Leininger, L., Harris, D., Tracz, S., and Marshall, J. (2013) Differences in physical activity participation between university employees with and without a worksite health promotion program, California Journal of Health Promotion 11, 67-75.

[5] Leininger, L., Orozco, B., and Adams, K. (2014) Worksite based walking competition: effects on perceived stress and physical activity in female university employees, Journal of Fitness Research 3 , $32-38$.

[6] Yang, X., Ge, C., Hu, B., Chi, T., and Wang, L. (2009) Relationship between quality of life and occupational stress among teachers, Public Health 121, 750-766. 
[7] Finkelstein, E. A., Fiebelkorn, I. C., and Wang, G. (2003) National medical spending attributable to overweight and obesity: how much, and who's paying?, Health Affairs Supp/ Web Exclusives, W3219-226.

[8] Tunceli, K., Bradley, C. J., Nerenz, D., Williams, L. K., Pladevall, M., and Elston Lafata, J. (2005) The impact of diabetes on employment and work productivity, Diabetes Care 28, 2662-2667.

[9] Harding, J., Freak-Poli, R. L., Backholer, K., and Peeters, A. (2013) Change in health-related quality of life amongst participants in a 4-month pedometer-based workplace health program, Journal of Physical Activity \& Health 10, 533-543.

[10] Yeo, T., Burrell, S., Sauter, P., Kennedy, E., Lavu, H., Leiby, B., and Yeo, C. (2012) A progressive post resection walking program significantly improves fatigue and health-related quality of life in pancreas and periampullary cancer patients, J Am Coll Surg 214, 463-475.

[11] Anokye, N. K., Trueman, P., Green, C., Pavey, T. G., and Taylor, R. S. (2012) Physical activity and health related quality of life, BMC Public Health 12, 624.

[12] Courneya, K. S., Mackey, J. R., Bell, G. J., Jones, L. W., Field, C. J., and Fairey, A. S. (2003) Randomized controlled trial of exercise training in postmenopausal breast cancer survivors: cardiopulmonary and quality of life outcomes, Journal of clinical oncology: Official Journal of the American Society of Clinical Oncology 21, 1660-1668.

[13] Ware, J., Kosinski, M., Turner-Bowker, D., and Gandek, B. (2002) User's manual for the SF-12v2 health survey with a supplement documenting SF-12 health survey, QualityMetric Incorporated, Lincoln, RI.

\section{ABOUT THE STUDENT AUTHORS}

Breanna Orozco will graduate from California State University, Monterey Bay in Spring 2015 with a Bachelor's degree in Kinesiology-Exercise Science and a Minor in Statistics. Breanna is a McNair scholar, and has received many other prestigious awards, including the American College of Sports Medicine Golding Scholarship. Each summer, Breanna travels to the University of Southern California to work in the Women's Health and Exercise Laboratory. She has presented at many national conferences including the American College of Sports Medicine Annual meetings, and the Hawaiian International Conference on Education. Her future endeavors include pursuing a Ph.D. in Biokinesiology and a career as a research professor.

Kendra Contente will graduate from California State University, Monterey Bay in Spring 2015 with a Bachelor's degree in Kinesiology - Exercise Science. Kendra has been involved in many projects at CSU Monterey Bay, including the annual Workplace Walkoff Challenge. Kendra will be presenting at several national conferences in the next year. Kendra is interested in health and exercise, and her main focus of research for the upcoming year will be interventions to increase physical activity among employees at universities. Kendra plans to pursue medical school.

\section{PRESS SUMMARY}

Research indicates that exercise can help improve health-related quality of life, which adds to the importance of participating in regular physical activity. Worksite health promotion programs aim to improve the health of employees, in an effort to reduce individual disease risk and benefit the organization by reducing health care costs. This study examined if quality of life could be improved among employees with participation in a walking competition at a university. This study did not find that quality of life was improved, however there are many other benefits to worksite health promotion programs, and therefore, studies should continue in this field. 


\title{
Approximation of Euler Number Using Gamma Function
}

\author{
Shekh Mohammed Zahid ${ }^{a *}$, and Dr. Prasanta Kumar Ray ${ }^{b}$ \\ a Institute of Mathematics and Applications, Bhubaneswar, Odisha, India \\ ${ }^{b}$ International Institute of Information Technology, Bhubaneswar, Odisha, India \\ *Student: shekhmohammedzahid@gmail.com \\ Mentor:prasanta@iiit-bh.ac.in
}

\section{ABSTRACT}

This research presents a formula to calculate Euler number using gamma function. The representation is somewhat similar to Taylor series expansion of $e$. The number $e$ is presented as sum of an integral and decimal part. But it is well known that $e$ is an irrational number, and therefore such an expression for $e$ does not exist in principle, that is why we are approximately representing it for use in computation. The approximation of our formula increases as we increase the value of index in the summation formula. We also analyse the approximation of our formula by both numerical and graphical methods.

\section{KEYWORDS}

Euler Number, Gamma Function, Approximation, Computing, L'Hospital's Rule

\section{INTRODUCTION}

The number $e$ is a mathematical constant and its value is approximately equal to 2.71828 , which is popularly known as Euler number or Napier constant. Its symbol is given in the honour of famous Swiss mathematician Leonhard Euler. The constant is not be confused with Euler-Mascheroni constant or simply Euler constant $\gamma^{1}{ }^{1}$ It is well known that the Euler number $e$ is irrational, that is, it cannot be represented as ratio of integers. Also it is transcendental, that is, it is not a root of any non-zero polynomial with rational coefficients. The number $e$ can be represented as a real number in many ways, as an infinite series, an infinite product, a continued fraction, or a limit of a sequence. The discovery of the constant $e$ is credited to Jacob Bernoulli, who tried to find the value of the given expression $\lim _{x \rightarrow \infty}\left(1+\frac{1}{x}\right)^{x}$, an expression that arises in the study of compound interest. It can also be calculated as the sum of the infinite series $\sum_{k=0}^{\infty} \frac{1}{k !}$. The number $e$ has many applications to probability theory, Newton's laws of cooling and in applied science.

In mathematics, the gamma function (represented by the Greek capital letter $\Gamma$ ) is an extension of the factorial function. The gamma function is defined for all complex numbers except the negative integers and zero. For complex numbers with a positive real part, it is defined via a convergent improper integral:

$$
\Gamma(n)=\int_{0^{+}}^{\infty} e^{-x} x^{n-1} d x, 0<\infty<n .
$$


If $0<n<1$, the integrand becomes infinite as $x \rightarrow 0^{+}$. The integral corresponding to the interval $(0,1)$ is convergent or proper for $0<n$, while that corresponding to the interval $(1, \infty)$ converges for all $n$. Hence, $\Gamma(n)$ is well defined integral for all $n>0 .^{2}$

The origin of gamma function is due to the problem of extending the factorial to noninteger arguments was apparently first considered by Daniel Bernoulli and Christian Goldbach in the 1720s, and was solved at the end of the same decade by Leonhard Euler. Euler gave two different definitions, first an infinite product of the form $n !=\prod_{k=1}^{\infty} \frac{\left(1+\frac{1}{k}\right)^{n}}{1+\frac{n}{k}} \quad$ and then he wrote to Goldbach again to announce his discovery of the integral representation $n !=\int_{0}^{1}(-\ln s)^{n} d s$ which is valid for $n>0$. By the change of variable $t=-\ln s$, this integral becomes the familiar Euler integral.Euler published these results in the paper "De progressionibus transcendentibus seu quarum termini generales algebraice dari nequeunt" “"On transcendental progressions, that is, those whose general terms cannot be given algebraically"). ${ }^{3}$

Some particular values of the gamma function are given in the following table:

\begin{tabular}{|c|c|}
\hline Value of $\boldsymbol{n}$ & Value of $\boldsymbol{\Gamma}(\boldsymbol{n})$ \\
\hline-1 & $\infty$ \\
\hline 0 & $\infty$ \\
\hline 1 & 1 \\
\hline$-3 / 2$ & $4 \sqrt{\pi} / 3$ \\
\hline$-1 / 2$ & $-2 \sqrt{\pi}$ \\
\hline $1 / 2$ & $\sqrt{\pi}$ \\
\hline $3 / 2$ & $\sqrt{\pi} / 2$ \\
\hline $5 / 2$ & $3 \sqrt{\pi} / 4$ \\
\hline
\end{tabular}

The gamma function has caught the interest of some of the most prominent mathematicians of all time. Its history, notably documented by Philip J. Davis in an article that won him the 1963 Chauvenet Prize, reflects many of the major developments within mathematics since the 18th century. In the words of Davis, "each generation has found something of interest to say about the gamma function. Perhaps the next generation will also." 4

\section{DERIVATION OF EULER NUMBER USING GAMMA FUNCTION}

In this section, we derive the Euler number using the notion of gamma function. We restrict the gamma function, $\Gamma(n)=\int_{0}^{\infty} e^{-x} x^{n-1} d x, 0<\mathrm{n}<\infty$ only to integral value of $n$. Replacing $n$ by $(n+1)$ and setting $x=1+a$ in the above equation we get,

$$
\Gamma(n+1)=\int_{-1}^{\infty} e^{-(1+a)}(1+a)^{n} d a
$$

Equation 1. 
Now expanding Equation 1

$$
\begin{aligned}
e \times \Gamma(n+1) & =\int_{-1}^{\infty} e^{-a}(1+a)^{n} d a \\
& =\int_{-1}^{0} e^{-a}(1+a)^{n} d a+\sum_{i=0}^{n-2}\left(\begin{array}{c}
n \\
i
\end{array}\right) \int_{0}^{\infty} e^{-a} a^{i} d a+\left(\begin{array}{c}
n \\
n-1
\end{array}\right) \int_{0}^{\infty} e^{-a} a^{n-1} d a+\left(\begin{array}{c}
n \\
n
\end{array}\right) \int_{0}^{\infty} e^{-a} a^{n} d a,
\end{aligned}
$$

Which on simplification gives

$e \times \Gamma(n+1)=\int_{-1}^{0} e^{-a}(1+a)^{n} d a+\sum_{i=0}^{\mathrm{n}-2}\left(\begin{array}{c}n \\ i\end{array}\right) \times \Gamma(i+1)+2 \times \Gamma(n+1) . \quad$ Equation 2.

Consider the function, $\xi(a)=e^{-a}(1+a)^{n}$ which is defined for all positive integers $n$. Now using Simpson's rule $\int_{\alpha}^{\beta} f(x) \approx \frac{\beta-\alpha}{6} \times\left(f(\alpha)+f(\beta)+4 \times\left(\frac{\alpha+\beta}{2}\right)\right) 5$ of numerical integration for approximation of definite integral of $\xi(a)$ from -1 to 0 , we obtain

$$
\begin{aligned}
\int_{-1}^{0} e^{-a}(1+a)^{n} d a & \approx\left(\frac{0-(-1)}{6}\right) \times(f(-1)+f(0)+4 \times f(-1 / 2)) \\
& \approx \frac{1}{6} \times\left(0+1+\frac{4 \times e^{1 / 2}}{2^{n}}\right) \approx \frac{1}{6}+\frac{e^{1 / 2}}{6 \times 2^{n-2}}
\end{aligned}
$$

which is approximately $\frac{0.2}{2^{n-2}}$. For larger values of $n$, the quantity $\frac{0.2}{2^{n-2}}$ will approach to zero. But for better approximation, the whole integral of the function $\xi(a)$ will be zero, as the quantity 0.1 is minute and adding it does not affect the integral. Thus, we have $\int_{-1}^{0} e^{-a}(1+a)^{n} d a \approx 0$

Therefore Equation 2 reduces to

$$
e \times \Gamma(n+1) \approx \sum_{i=0}^{n-2}\left(\begin{array}{c}
n \\
i
\end{array}\right) \times \Gamma(i+1)+2 \times \Gamma(\mathrm{n}+1) .
$$

Using the property $\Gamma(t+1)=t !^{2}$, we get

$$
e \times n ! \approx \sum_{i=0}^{n-2}\left(\begin{array}{c}
n \\
i
\end{array}\right) \times i !+2 \times n !
$$

which on simplification gives

$$
e \approx \sum_{i=0}^{n-2} \frac{1}{(n-i) !}+2, \text { where } n \geq 2
$$

Equation 3.

\section{AJUR Volume 12 | Issue 3 | May 2015}


The following table represents the values of the Euler number $e$ for different values of $n$ using Equation 3 .

\begin{tabular}{|c|c|}
\hline Value of $n$ & Value of $e$ \\
\hline 2 & 2.5 \\
\hline 3 & 2. 66666666666666667 \\
\hline 4 & 2.7083333333333333 \\
\hline 5 & 2.71666666666666667 \\
\hline 6 & 2.71805555555555556 \\
\hline 7 & 2.7182539682539683 \\
\hline 8 & 2.7182787698412698 \\
\hline 9 & 2.7182815255731922 \\
\hline 10 & 2.7182818011463845 \\
\hline 11 & 2.7182818261984929 \\
\hline 12 & 2.7182818282861686 \\
\hline 13 & 2.718281828446759 \\
\hline 14 & 2. 7182818284582297 \\
\hline 15 & 2.7182818284589945 \\
\hline 16 & 2.7182818284590423 \\
\hline 17 & 2.7182818284590451 \\
\hline 18 & 2.7182818284590452 \\
\hline 19 & 2.7182818284590452 \\
\hline 20 & 2.7182818284590452 \\
\hline
\end{tabular}

\section{ANALYSIS OF APPROXIMATION}

In this section, the approximation of the Euler number $e$ given in Equation 3 is analysed numerically and graphically.

\section{Numerical Method}

It is observed that by substituting $x=1+a$ in Equation 1, the limit changed from -1 to $\infty$, but the actual upper limit was $\infty-1$. Also we approximated the integral of $\xi(a)$ from -1 to 0 to zero. This brings an approximation in Equation 3.

The next graphical approach emphasizes the cause of approximation more clearly.

\section{Graphical Method}

Differentiating the function $\xi(a)=e^{-a}(1+a)^{n}$ with respect to $a$ and computing $\xi(a)$ at -1 and 0 , we get

$$
\xi(-1)=0, \xi(0)=1, \frac{d \xi(a)}{d a}=e^{-a}(1+a)^{n-1}(n-(a+1))
$$


To compute the limit of $\xi(a)$ when $a \rightarrow \infty$, we expand the expression $(1+a)^{n}$ by binomial theorem and evaluate the limit of each term using L'Hospital's rule, since each term (excluding first term) is in $\frac{\infty}{\infty}$ form. Further, differentiating each term (excluding first term) to numbers of times the respective power of ' $a$ ', as $e^{a}$ never vanishes, all terms will be in $\frac{1}{\infty}$ form. Therefore we get

$$
\lim _{a \rightarrow \infty} \frac{\left(\sum_{k=0}^{n}\left(\begin{array}{l}
n \\
k
\end{array}\right) a^{k}\right)}{e^{a}}=0
$$

Equation 4.

Now we observe the graphs of $\int_{-1}^{\infty} e^{-a}(1+a)^{n} d a$ for different values of $n$

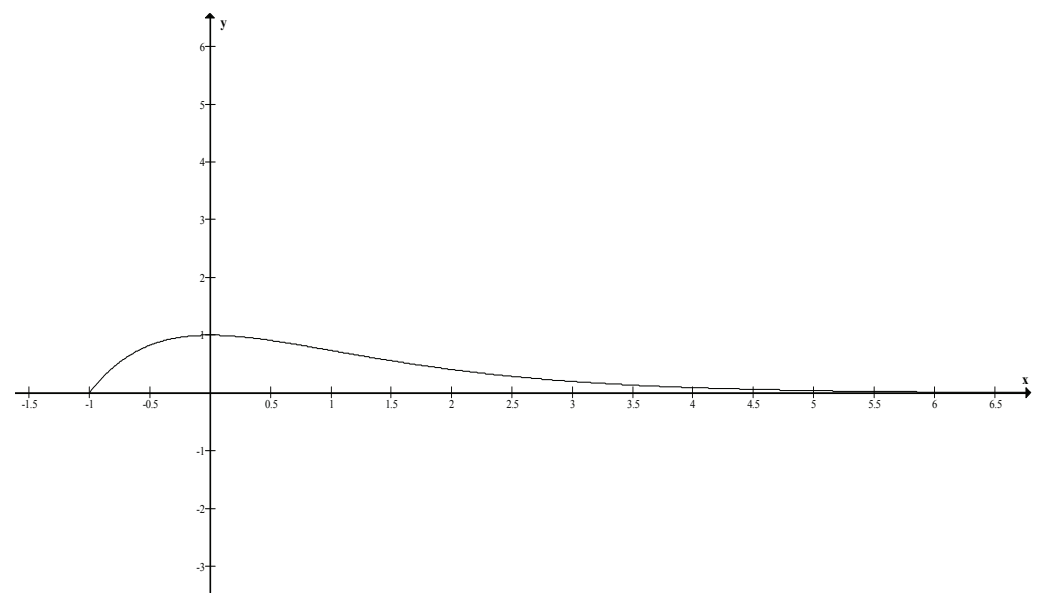

Figure 1. $n=1$

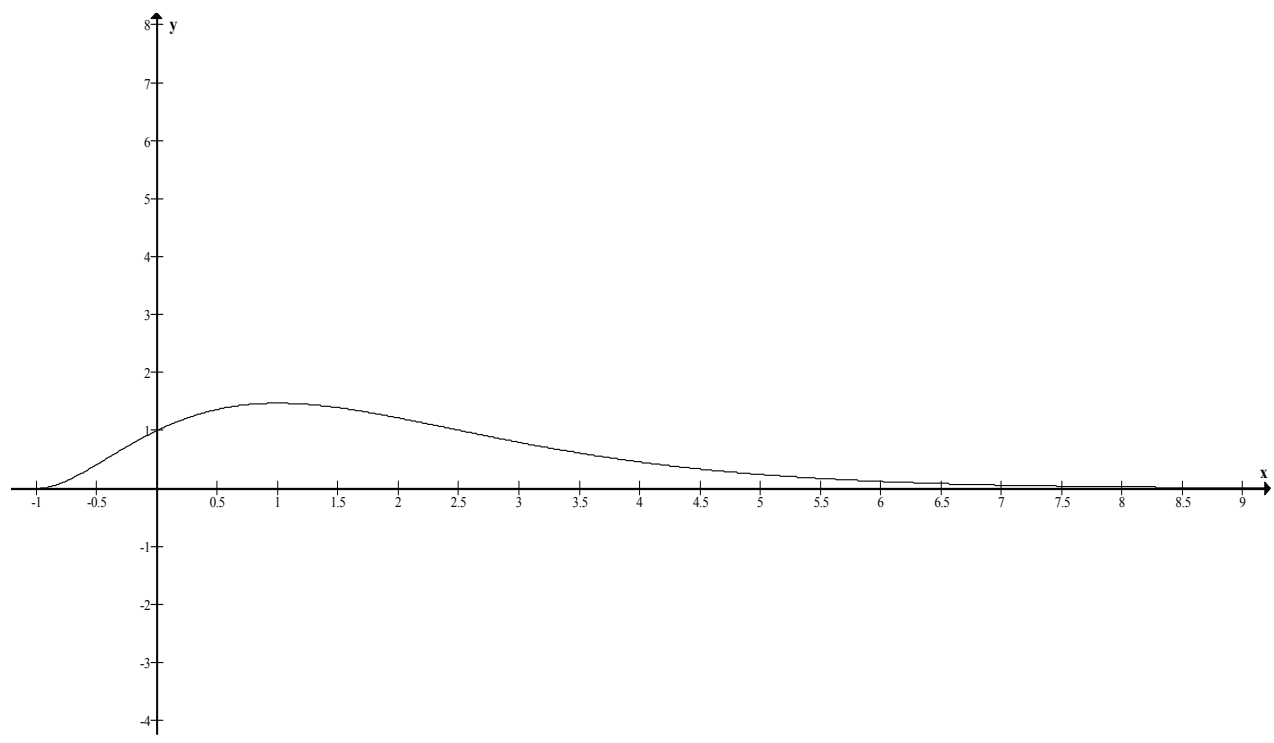

Figure 2. $n=2$ 


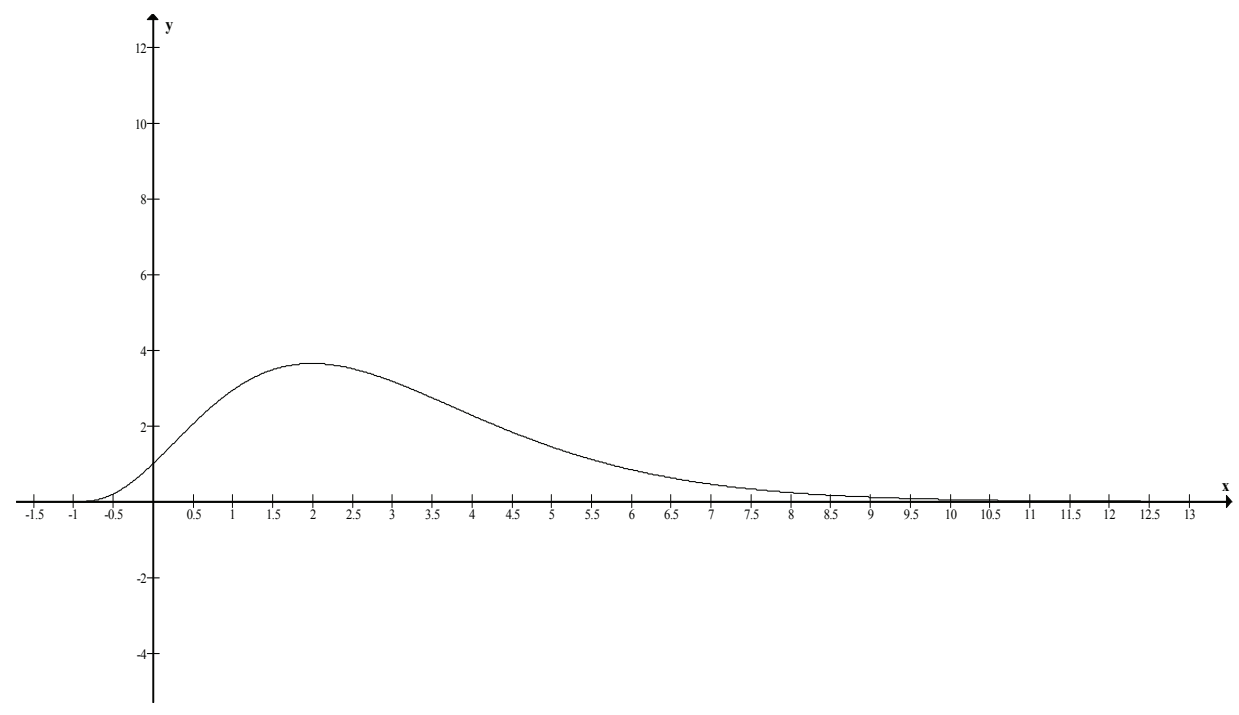

Figure 3. $n=3$

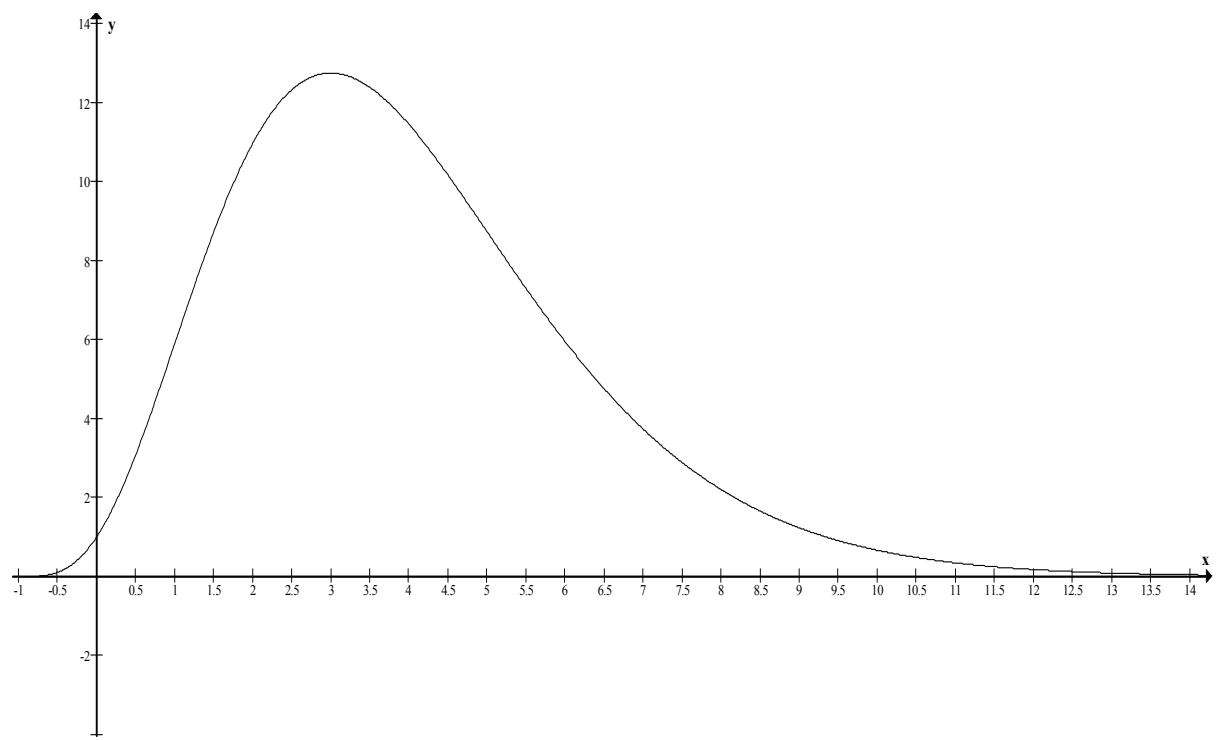

Figure 4. $n=4$ 


\section{ANALYSIS OF GRAPHS}

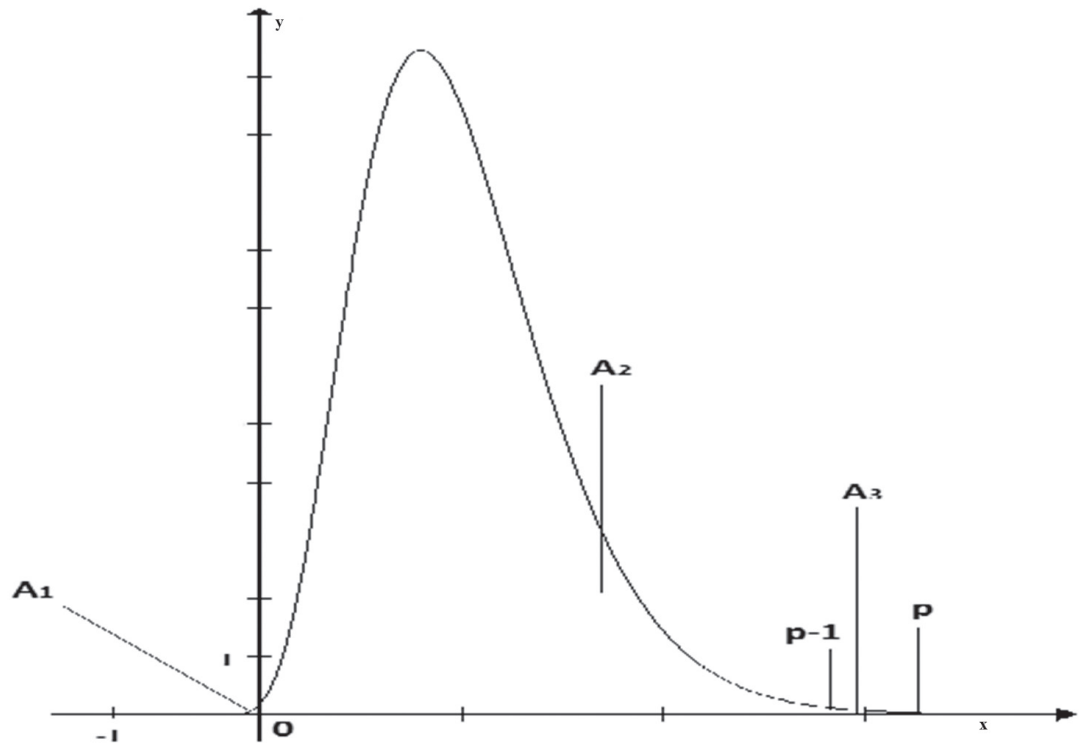

Figure 5. Rough graph of Figure 1, Figure 2, Figure 3 and Figure 4.

Analysing Figure 5, we observe that as the value of $n$ increases the steepness of graph between -1 and 0 towards origin increases which means the graph is more approaching towards origin. Also the concave graph between 0 to $\infty$ is going more upward. In the above figure, we observe that the area $A_{\text {Total }}=A_{1}+A_{2}$, where $A_{1}$ and $A_{2}$ are area of regions as shown in the above Figure 5.

Using Simpson rule for approximating the integrals and using Equation 4, we obtain

$\int_{p-1}^{p} e^{-a}(1+a)^{n} d a \approx\left(\frac{p-(p-1)}{6}\right) \times\left(f(p-1)+f(p)+4 \times f\left(\frac{p-1+p}{2}\right)\right) \approx \frac{1}{6} \times(f(p)+f(p)+4 \times f(p))$.

As $p \rightarrow \infty, p-1 \rightarrow \infty$ and also $2 p-1 \rightarrow \infty$. Therefore, we have

$$
\int_{p-1}^{p} e^{-a}(1+a)^{n} d a \approx f(p) \approx \lim _{p \rightarrow \infty} \frac{(1+p)^{n}}{e^{p}} \approx 0
$$

The integral $\mathrm{A}_{3}=\int_{p-1}^{p} e^{-a}(1+a)^{n} d a$ and the integral of $\xi(a)$ from -1 to $0, \mathrm{~A}_{1}$, both are tending to same value zero, therefore we must have $A_{1} \approx A_{3}$. Hence replacing area $A_{1}$ by area $A_{3}$, we have $\mathrm{A}_{\text {Total }} \approx \mathrm{A}_{2}+\mathrm{A}_{3}$.

\section{CONCLUSION}

Though the Euler number is evaluated using Taylor series, in this study, we have given an alternative method of it to approximate the Euler number using gamma function. We have also connected the graphs with the mathematical equation and observe how the curve varies to fulfil the approximation process. 


\section{REFERENCES}

[1] Maor, E. (2009), e: The Story of a Number, Princeton University Press. pp-28-38.

[2] Widder, D.V. (2013), Advanced Calculus, Second edition, PHI Learning Private Limited, pp-367-378.

[3] Sandifier, C. E. (2007), The Early Mathematics of Leonhard Euler constant, Mathematical Association of America, pp-33-43.

[4] Mathematical Association of America (2008), Leonhard Euler's Integral: A Historical Profile of the Gamma Function, American Mathematical Monthly, from http://www.maa.org/programs/ maa-awards/writing-awards/leonhard-eulers-integral-an-historical-profile-of-the-gamma-function.

[5] Maron, I.A. (1973), Problems in Calculus of One Variable, CBS Publishers and Distributors, pp-301-307.

\section{ACKNOWLEDGMENTS}

I truly thank my parents for support and my mentor Dr. Prasanta Kumar Ray, IIIT, Bhubaneswar for helping me to review this paper.

\section{ABOUT THE STUDENT AUTHOR}

Shekh Mohammed Zahid is a first year undergraduate student of BSc (Hons) in Mathematics and Computing in the Institute of Mathematics and Applications, Bhubaneswar, Odisha, India. His research interests include number theory and theoretical physics.

\section{PRESS SUMMARY}

In this paper, we developed a formula that gives an approximate value of the Euler number $e$ using gamma function. Its expansion is the sum of integer part and the fractional part. Also, the expansion of series can be terminated as per our preference by choosing favourable index in the summation formula. We have approximated the Euler number $e$ in the given formula for a specific value; however, there is a scope to extend the formula for approximating $e$ in a new way using asymptotic notation. 


\title{
Direct and Indirect Effects of Pseudoephedrine on the Intrinsic Conduction System of the Embryonic Chicken Heart
}

\author{
Samely Gonzalez, Fatima Afzal and Dr. Jacqueline S. McLaughlin \\ Department of Biology, Penn State Lehigh Valley, Center Valley, PA \\ Students: gnzlz_smly2@yahoo.com,fia5083@psu.edu \\ Mentor: jxm57@psu.edu
}

\begin{abstract}
Pseudoephedrine (PSE) is an over the counter (OTC) medication used to temporarily relieve nasal congestion, hay fever or other upper respiratory allergies by shrinking blood vessels in the nasal passages. Aside from its vasoconstriction properties it is also known to elevate heart rate, and blood pressure, thus being a sympathomimetic drug. There are two hypotheses on how this drug increases heart rate (HR): (1) a direct mechanism wherein PSE works by binding to adrenergic receptors in the heart's intrinsic conduction system; and, (2) an indirect mechanism wherein PSE causes the release of norepinephrine from sympathetic nerves to activate adrenergic receptors. This research utilized the chick embryonic heart as a model system to examine the chronotropic effects and mechanisms of PSE on the developing vertebrate heart. Research suggests that this drug has both direct and indirect effects, and induces dangerous heart arrhythmias such as atrial flutter, in high doses.
\end{abstract}

\section{KEYWORDS}

Pseudophedrine, Sympathomimetic Drugs, Pseudophedrine and Pregnancy, Pseudophedrine and Norepinephrine, Pseudophedrine Related Arrhythmias, Over-the-counter Drugs; Chick Heart Development

\section{INTRODUCTION}

Psuedoephederine (PSE) is the active ingredient in many Food and Drug Administration (FDA)-approved nasal decongestant products, including Sudafed ${ }^{\circ}$. Despite its wide distribution in local pharmacies and its common use, Sudafed ${ }^{\circ}$ has become a topic of interest in drug research due to its numerous side effects on the cardiovascular system such as increased heart rate. The autonomic regulation of $\mathrm{HR}$ relies on two branches of the autonomic nervous system, sympathetic and parasympathetic, which have an impact on the electrical impulses and the timing that are generated from the SA node. ${ }^{4}$ The sympathetic division speeds up pacemaker's depolarization via the exocytosis of norepinephrine (NE), while the parasympathetic division slows it down by releasing acetylcholine. Even though this drug has been proven to affect the cardiovascular system, it is unclear whether it works on the heart via a direct or indirect mechanism. The two hypotheses regarding these mechanisms are the following: (1) A direct mechanism wherein PSE works by binding to adrenergic receptors in the heart's intrinsic conduction system; and, (2) an indirect mechanism of action is via stimulating the release of NE from presynaptic nerve endings which then freely activate $\alpha$ - and $\beta$-adrenergic receptors located within the SA node and the muscle lining walls of blood vessels. The adrenergic receptors are a class of $\mathrm{G}$ protein-coupled receptors that bind agonists such as catecholamines to generally stimulate the sympathetic nervous system. Activation of the beta receptor leads to increases in contractile force and heart rate. Activation of alpha receptors by either sympathetic nervous system transmission or drugs will result in vasoconstriction and an increase in peripheral resistance and systemic arterial blood pressure. ${ }^{5}$ It is noteworthy to mention that the parasympathetic nervous system works through a different set of receptors not $\alpha$ - and 
$\beta$-adrenergic receptors.

Studies on anesthetized adult rats showed that PSE causes dose-dependent increases in arterial blood pressure and heart rate (HR). The increase in HR disappeared after the destruction of the sympathetic nerve terminals, suggesting that PSE's mechanism of action is indirect and innervation is needed to affect the HR. ${ }^{6}$ This rules out a direct effect because after the termination of the sympathetic nerves, the receptors on the heart were still present and could technically still be bound by PSE to increase the HR; however, the heart rate no longer increased even though the receptors were exposed to the drug, suggesting the inability of PSE to directly bind to the receptors. Based on these findings, it was hypothesized that PSE will exhibit dose-dependent chronotropic effects on the in vitro 6-day intact chick embryo, but not on an in vitro denervated 6-day chick heart. It was reasoned that the 6 -day isolated heart without innervation will not have the capability to respond to this drug due to the disruption of sympathetic nerve input.

Avian organisms are commonly used as model systems for the study of the cardiovascular system in humans as both systems share a four-chambered heart. During bird and human heart development, endothelial tubes fuse to form four distinct regions: ventricle, atrium, conotruncus (aka bulbus cordis), and the sinus venosus. The latter functions as the embryonic pacemaker. Once the four-chambered heart is developed, between days 6-7, the sinus venosus becomes the mature pacemaker or sinoatrial node (SA node). ${ }^{1,2,3}$ The SA node is a cluster of auto-rhythmic cells located in the wall of the right atrium that sets the rate and timing at which cardiac muscle cells contract. However, the adrenergic receptors located at the SA node are influenced by neurotransmitters or sympathomimetic drugs, therefore changing the normal auto-rhythm of the SA node cells. Overall, this research on the fetal heart system provides an understanding of sympathomimetic drugs and the adrenergic receptors at which these drugs interact. Understanding and awareness of such drugs is critical because they are widely used by the general public as a nasal decongestant and by medical professionals to treat cardiac arrest, and low blood pressure, among other things.

\section{METHODS AND MATERIALS}

\section{A. Serial Dilution}

A 10-fold serial dilution of PSE was prepared using a PSE stock solution of $3 \mathrm{mg} / \mathrm{mL}$ in CMRL $10661 \mathrm{X}$ Media (Mediatech Inc., Manassas, VA) to yield the following concentrations: $3 \times 10^{-1} \mathrm{mg} /$ $\mathrm{mL}, 3 \times 10^{-2} \mathrm{mg} / \mathrm{mL}, 3 \times 10^{-3} \mathrm{mg} / \mathrm{mL}, 3 \times 10^{-4} \mathrm{mg} / \mathrm{mL}$, and $3 \times 10^{-5} \mathrm{mg} / \mathrm{mL}$.

\section{B. Windowing the Egg - $\mathrm{Cruz}^{7}$}

A 6-day fertilized broiler egg was removed from a $41^{\circ} \mathrm{C}$ egg incubator into a $110 \mathrm{~mm}$ glass dish lined with cotton, maintaining its horizontal position. While keeping the egg warm under a goose neck lamp with $100 \mathrm{~W}$ bulb, Scotch ${ }^{\oplus} \mathrm{Magic}^{\mathrm{mi}}$ tape was placed along the long axis of the egg. A dissecting needle was used to puncture a hole in the blunt end of the egg. Then, a $20 \mathrm{G}$ needle with syringe was inserted into the hole to remove approximately $1-2 \mathrm{~mL}$ of albumen. Next, an oval opening was cut with micro-dissecting scissors, exposing the embryo and its extra-embryonic membranes.

\section{Explanting the Embryo - Cruz}

Using an embryo spoon and micro-dissecting scissors, the extra-embryonic membranes were cut to explant the embryo into a $65 \mathrm{~mm}$ Syracuse glass dish containing warmed CMRL media on the stage of a dissecting microscope. The chick explant was oriented so the right side was facing upward to allow access to the beating heart. 


\section{Heart Isolation - McLaughlin and McCain ${ }^{8}$}

The beating heart was removed with surgical scissors and fine forceps by cutting it above the conotruncus and below the SA node or atrium. The excised heart was transferred into a Bioptechs EDU Series Culture Dish Control System (Butler, PA), attached to a dissecting microscope, to maintain normal avian body temperature at $41^{\circ} \mathrm{C}$. Its control $\mathrm{HR}$ (beats per minute) was then visually assessed and recorded without drug. HR was recorded in three intervals of 15 seconds each, using a stopwatch, and then averaged.

\section{E. Application of Pseudoephedrine}

The isolated hearts were suspended in five PSE concentrations starting with lowest $\left(3 \times 10^{-5} \mathrm{mg} /\right.$ $\mathrm{mL})$ to highest $\left(3 \times 10^{-1} \mathrm{mg} / \mathrm{mL}\right)$. Previous concentrations of drug were removed with a transfer pipette properly labeled as drug with labeling tape and indelible ink pen. HRs and arrhythmias were recorded for each concentration following an approximate 30 second period of equilibration. For whole embryos, the in vitro HR was recorded without the drug (control) and again in each PSE concentration from lowest to highest (Figures 1 and 2). Any heart arrhythmias that were observed were also recorded.

\section{F. Statistical Analysis}

Polynomial regression analysis was chosen to represent the non-linear trend in the data points between the varying concentrations of PSE and HR. Furthermore, a second order polynomial trend line is selected to set the data on the graph to best address the number of fluctuations. The second-order quadratic equation is represented in quadratic form, $y=a x^{2}+b x+c$. In Figure 4, error bars represent one standard deviation above and below the mean $(\mathrm{SD} \pm 1)$ for both the isolated heart and whole embryo trials. To clarify, the mean of trials 1-3 was calculated for both the isolated hearts and whole embryos separately, and then the custom STDEV.S function in MS Excel was applied to calculate the error bars (note: this function calculates the standard deviation of a sample, rather than of a larger population). The more the raw data values lie above and below the mean, the wider the error bars and less confident one can be in a particular value. If the upper error bar for one drug concentration overlaps the range of values within the error bar of another drug concentration, there is a much lower likelihood that these two values differ significantly. ${ }^{9}$
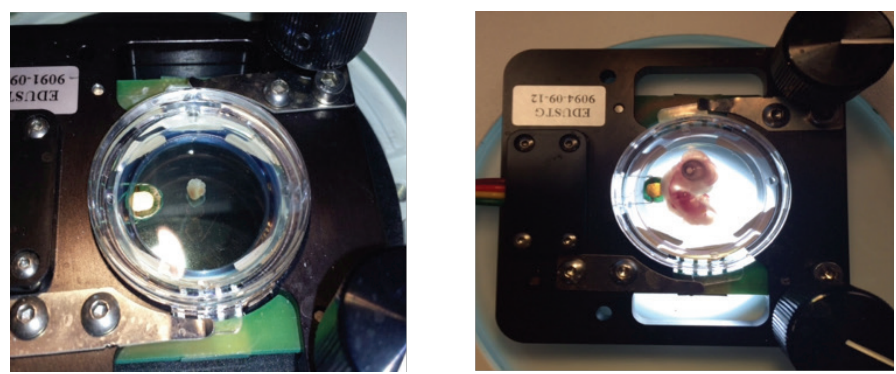

Figure 1. Delta T-EDU Bioptechs apparatus illustrating a 6-day isolated chick heart. (left)

Figure 2. Delta T-EDU Bioptechs apparatus illustrating a 6-day whole chick embryo. (right) 


\section{RESULTS}

Polynomial regression analysis was used to interpret the relationship between HRs and increasing PSE concentrations. Three experimental trials were carried out, each consisting of an isolated 6-day chick heart and a whole 6-day chick embryo. Additionally, polynomial trend lines were used to best present the overall trends as data fluctuated between concentrations. Figure $3(\mathbf{a}, \mathbf{b}, \mathbf{c})$ presents polynomial regressions that plot HR vs. PSE concentrations for all three trials. Control data is also plotted on the graphs, which reflect HR of isolated heart and whole embryo without drug (CMRL media only). Additionally, Tables $\mathbf{1}$ and $\mathbf{2}$ display the average HR values for the isolated hearts and whole embryos along with observations of arrhythmias per concentrations. The isolated heart 1 and whole embryo 1 data shown in the tables below make up the first trial, isolated heart 2 and whole embryo 2 are compared as the second trial, and isolated heart 3 and whole embryo 3 were compared as the third trial. Overall, the following consistent pattern is seen throughout all three trials; an increase in HR towards the mid-concentration of $3 \times 10^{-1} \mathrm{mg} / \mathrm{mL}$ followed by a gradual decrease in HR towards the highest concentration of $3 \times 10^{-1} \mathrm{mg} / \mathrm{mL}$. Tachycardia was most prevalent at lower concentrations followed by atrial flutter and bradycardia towards the highest concentrations, in which some cases lead to cardiac arrest. For example, the isolated heart of trial 1 showed a control HR of 125 beats per minute (bpm) which then increased to $188 \mathrm{bpm}$ at the concentration of $3 \times 10^{-3} \mathrm{mg} / \mathrm{mL}$, illustrating tachycardia. This accelerated HR then fell to $73 \mathrm{bpm}$ at the highest concentration at $3 \times 10^{-1} \mathrm{mg} / \mathrm{mL}$, therefore bradycardia was observed along with atrial flutter.

Figure 4 illustrates a graphical presentation of the average HRs of 6-day isolated hearts and whole embryos exposed to varying concentrations of PSE (the three trials presented above as well as the control data without drug). The polynomial regression line models the same overall trend between the average HRs and PSE concentrations, revealing a polynomial increase in HR up until $3 \times 10^{-3}$ $\mathrm{mg} / \mathrm{mL}$, followed by a gradual decrease until the highest concentration, $3 \times 10^{-1} \mathrm{mg} / \mathrm{mL}$. Standard error bars were determined to evaluate the uncertainty around the estimate of the mean measurement. Standard error is used as a measurement of precision of the sample mean, and depends largely on both standard deviation and sample size. The error bars that are displayed in Figure 4 represent one standard deviation above and below the expected HR values plotted for each drug concentration. The average HR data indicates a $68 \%$ confidence interval of the data set with respect to the mean. Clearly, the distance between the two means for each concentration overlaps. This suggests statistical insignificance.

It is noteworthy to mention that aside from recording HRs, meticulous observations were made on the sinus rhythm of the chick hearts exposed to various PSE concentrations. The isolated chick hearts and whole chick embryos exposed to the drug experienced many arrhythmias. After the control media was removed and the lowest concentration was added, episodes of tachycardia were the most prevalent arrhythmias observed. However, at the highest concentrations of PSE, $3 \times 10^{-1}$ $\mathrm{mg} / \mathrm{mL}$, bouts of atrial flutter, bradycardia, and cardiac arrest were the most predominant effects. In a developing vertebrate heart tachycardia can be categorized as a sinus rhythm over $160 \mathrm{bpm}$, while bradycardia describes a sinus rhythm in which the heart rate is around $90 \mathrm{bpm}$. As for atrial flutter the heart rate in the atrium is usually very fast, often in the range of 350-500 beats per minute. Fortunately, the AV node is usually incapable of transferring impulses at such a fast rate, so only every other or every third impulse is conducted to the ventricles, this result in a heart rate in the ventricles that is much slower than the atrium, but still usually faster than normal. 


\begin{tabular}{|c|c|c|c|c|c|c|}
\hline Concentrations & $\begin{array}{l}\text { Isolated } \\
\text { Heart } 1\end{array}$ & Observations & $\begin{array}{l}\text { Isolated } \\
\text { Heart } 2\end{array}$ & Observations & $\begin{array}{l}\text { Isolated } \\
\text { Heart } 3\end{array}$ & Observations \\
\hline \multirow{2}{*}{ No drug } & Avg. & \multirow{2}{*}{$\begin{array}{l}\text { Normal sinus } \\
\text { rhythm }\end{array}$} & Avg. & \multirow{2}{*}{$\begin{array}{c}\text { Tear in atria; } \\
\text { normal sinus } \\
\text { rhythm }\end{array}$} & Avg. & \multirow{2}{*}{$\begin{array}{l}\text { Normal sinus } \\
\text { rhythm }\end{array}$} \\
\hline & 125 & & 156 & & 105 & \\
\hline $3 \times 10^{-5} \mathrm{mg} / \mathrm{mL}$ & 100 & $\begin{array}{l}\text { Normal sinus } \\
\text { rhythm }\end{array}$ & 140 & Atrial flutter & 115 & $\begin{array}{l}\text { Normal sinus } \\
\text { rhythm }\end{array}$ \\
\hline $3 \times 10^{-4} \mathrm{mg} / \mathrm{mL}$ & 188 & Tachycardia & 137 & Atrial flutter & 177 & Tachycardia \\
\hline $3 \times 10^{-3} \mathrm{mg} / \mathrm{mL}$ & 188 & Tachycardia & 165 & Atrial flutter & 188 & Tachycardia \\
\hline $3 \times 10^{-2} \mathrm{mg} / \mathrm{mL}$ & 171 & Tachycardia & $\mathrm{CA}$ & $\begin{array}{l}\text { Cardiac arrest } \\
\text { (CA) }\end{array}$ & 134 & $\begin{array}{c}\text { Periods of } \\
\text { tachycardia and } \\
\text { bradycardia }\end{array}$ \\
\hline $3 \times 10^{-1} \mathrm{mg} / \mathrm{mL}$ & 73 & $\begin{array}{l}\text { Atrial flutter; } \\
\text { bradycardia }\end{array}$ & $\mathrm{CA}$ & CA & 97 & $\begin{array}{c}\text { Bradycardia; } \\
\text { periods of atrial } \\
\text { flutter }\end{array}$ \\
\hline
\end{tabular}

Table 1. Average of heart rates (HRs) in beats per minute (bpm) of three isolated hearts with respects to increasing pseudoephedrine (PSE) concentrations.

\begin{tabular}{|c|c|c|c|c|c|c|}
\hline Concentrations & $\begin{array}{c}\text { Whole } \\
\text { Embryo } 1\end{array}$ & Observations & $\begin{array}{c}\text { Whole } \\
\text { Embryo } 2\end{array}$ & Observations & $\begin{array}{c}\text { Whole } \\
\text { Embryo } 3\end{array}$ & Observations \\
\hline \multirow{2}{*}{ No drug } & Avg. & \multirow{2}{*}{$\begin{array}{l}\text { Normal sinus } \\
\text { rhythm }\end{array}$} & Avg. & \multirow{2}{*}{$\begin{array}{l}\text { Normal sinus } \\
\text { rhythm }\end{array}$} & Avg. & \multirow{2}{*}{$\begin{array}{l}\text { Normal sinus } \\
\text { rhythm }\end{array}$} \\
\hline & 128 & & 121 & & 120 & \\
\hline $3 \times 10^{-5} \mathrm{mg} / \mathrm{mL}$ & 91 & Bradycardia & 143 & $\begin{array}{l}\text { Normal sinus } \\
\text { rhythm }\end{array}$ & 156 & $\begin{array}{l}\text { Normal sinus } \\
\text { rhythm }\end{array}$ \\
\hline $3 \times 10^{-4} \mathrm{mg} / \mathrm{mL}$ & 124 & $\begin{array}{l}\text { Normal sinus } \\
\text { rhythm }\end{array}$ & 153 & $\begin{array}{l}\text { Normal sinus } \\
\text { rhythm }\end{array}$ & 170 & Tachycardia \\
\hline $3 \times 10^{-3} \mathrm{mg} / \mathrm{mL}$ & 136 & $\begin{array}{l}\text { Normal sinus } \\
\text { rhythm }\end{array}$ & & $\begin{array}{l}\text { No data } \\
\text { (lab error) }\end{array}$ & 183 & Tachycardia \\
\hline $3 \times 10^{-2} \mathrm{mg} / \mathrm{mL}$ & 91 & Bradycardia & 170 & Tachycardia & $\mathrm{CA}$ & $\begin{array}{l}\text { Bradycardia; } \\
\text { atrial flutter }\end{array}$ \\
\hline $3 \times 10^{-1} \mathrm{mg} / \mathrm{mL}$ & 75 & $\begin{array}{l}\text { Bradycardia; } \\
\text { atrial flutter }\end{array}$ & 36 & $\begin{array}{l}\text { Bradycardia; } \\
\text { atrial flutter }\end{array}$ & $\mathrm{CA}$ & Cardiac arrest \\
\hline
\end{tabular}

Table 2. Average of heart rates (HRs) in beats per minute (bpm) of three whole embryos with respect to increasing pseudoephedrine (PSE) concentrations. 


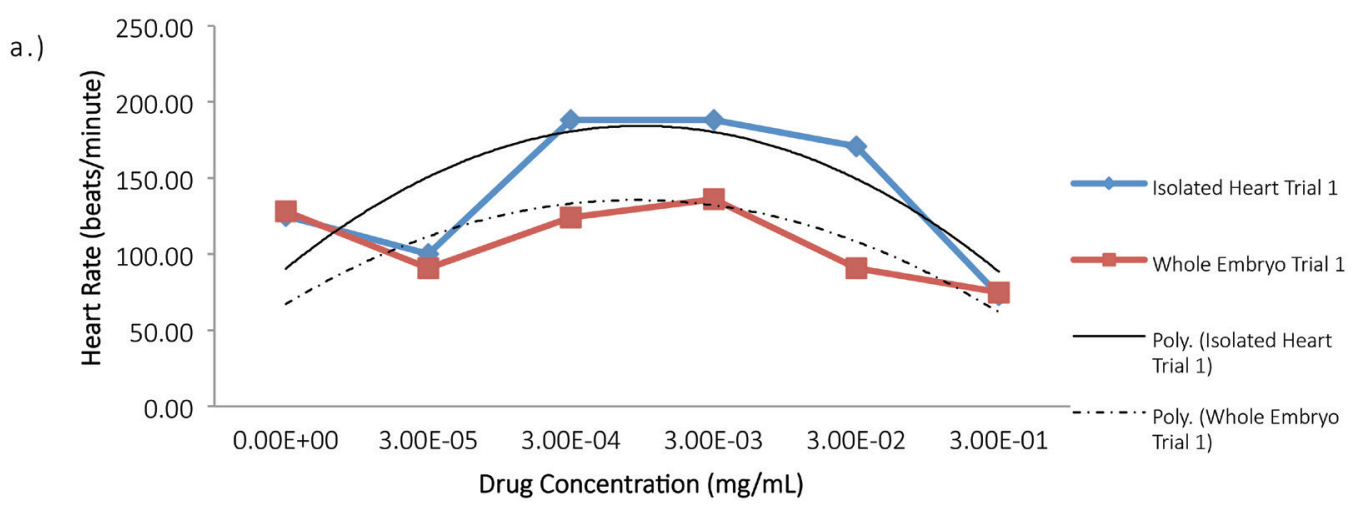

b.
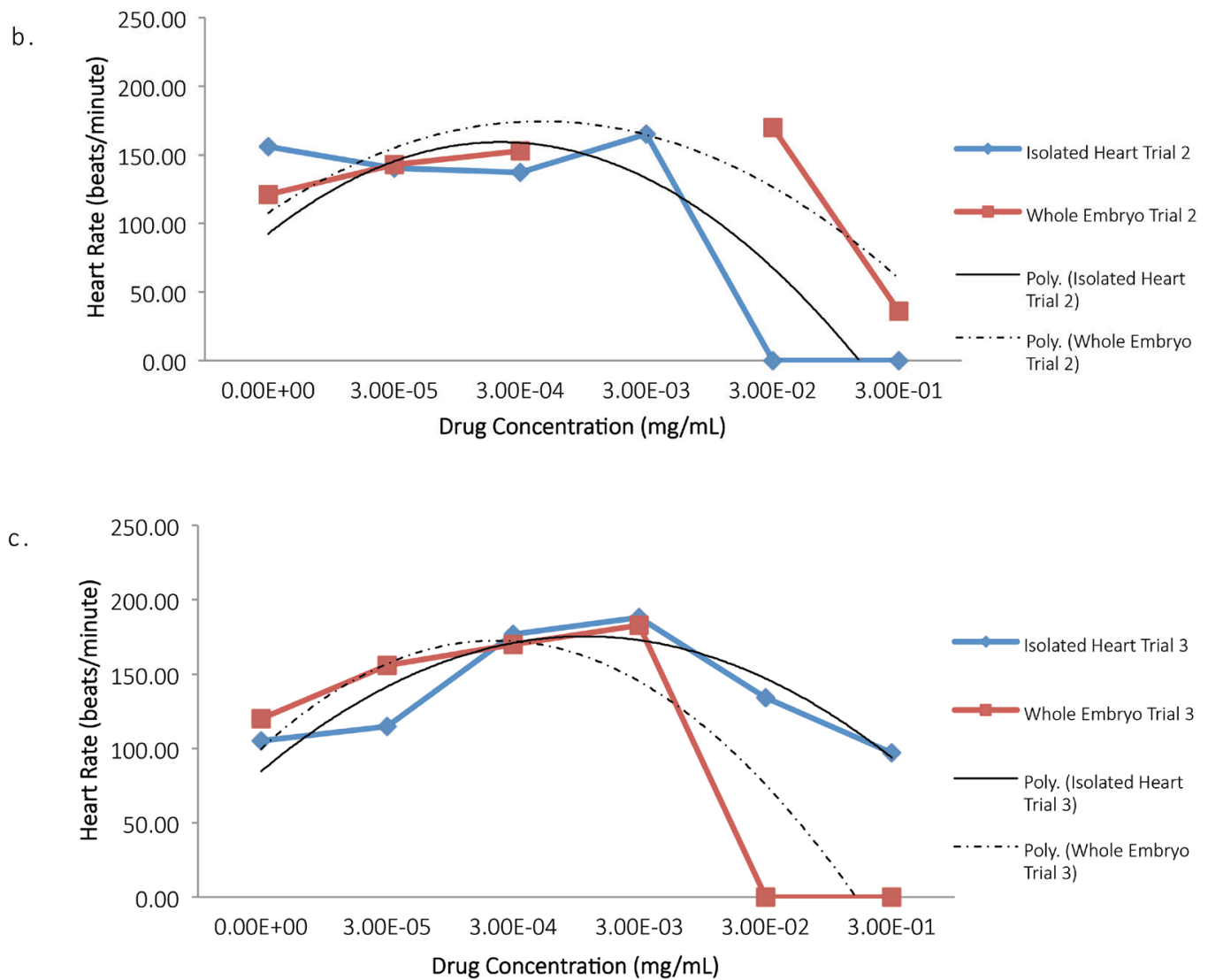

Figure 3. a, b, c. Polynomial Regression Curves illustrating HR of a 6-day isolated chick heart (blue) and whole embryo (red) exposed to varying concentrations of PSE. CMRL was used as the control (no drug). Figure 3 b., data for drug concentration $3.00 \mathrm{E}-03 \mathrm{mg} / \mathrm{mL}$ was not recorded. 


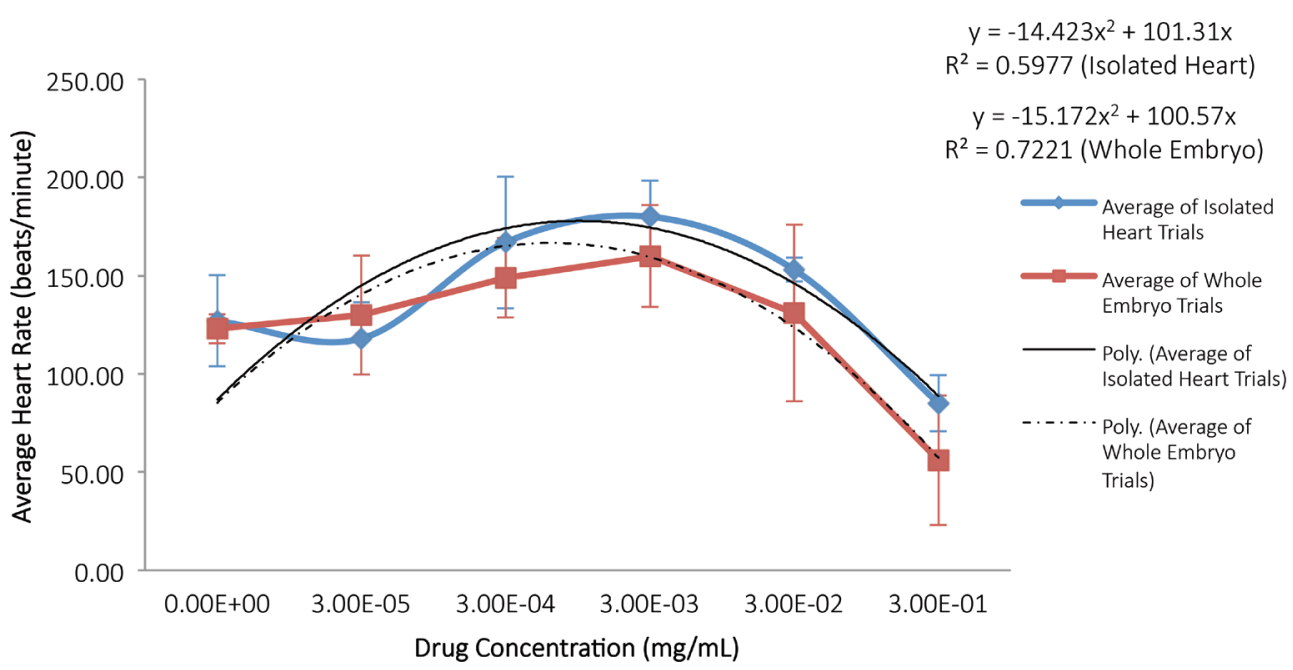

Figure 4. Polynomial regression representing average HR of 6-day isolated chick hearts (blue) and whole embryos (red) exposed to varying concentrations of PSE. CMRL was the control (no drug). Error bars represent the mean \pm 1 SD, for both the isolated hearts, in blue $(n=3)$ and the whole embryos, in red $(n=3)$.

\section{DISCUSSION}

The pharmacokinetics and pharmacodynamics of PSE are fairly well understood in various vertebrate model systems; however, no information exists on the effects of this drug on a vertebrate embryo. Not only did the experiment presented in this research paper intend to determine if PSE worked directly and/or indirectly on a fetal heart system, by utilizing five different PSE concentrations (lowest to highest), this research was also able to illustrate the dose-response relationship between this drug and its effects on the heart. It is important to note that around the lowest concentration, both isolated hearts and whole embryos showed normal sinus rhythm but increasing the doses of PSE caused abnormal changes in the electrical activity of the heart. The drug increased the HR to the point of tachycardia followed by atrial flutter and bradycardia at highest concentrations. In regards to this drug's mechanism of action, data suggests the following: both 6-day in vitro "isolated" chick hearts and whole chick embryos exposed to PSE showed the capability to respond to this drug. Based on the data, in both the isolated and whole embryo systems, the HR increased with increasing concentrations; however, at the highest concentrations, $3 \times 10^{-2} \mathrm{mg} / \mathrm{mL}$ and $3 \times 10^{-1}$ $\mathrm{mg} / \mathrm{mL}, \mathrm{HR}$ showed a copious fall along with various episodes of arrhythmias, especially atrial flutter. According to previous research conducted on anesthetized rats, PSE caused dose-dependent increases in HR, but after using the drug 6-hydroxydopamine (6-ODHA) for the destruction of sympathetic nerve terminals, these effects on the HR disappeared, suggesting that PSE's effect on HR was dependent on the presence of these nerves, i.e., an indirect effect. ${ }^{6}$ From these results in a fully developed vertebrate system, we reasoned that PSE would only increase the HR of the whole chick embryo with intact innervation (indirect effect), and not that of the isolated chick heart (direct effect). This was reasoned because previous research suggested that stimulation of PSE worked solely through the interaction between the sympathetic nervous system and the heart. However, the isolated heart no longer had external nerve supply which impaired the response to manipulations by the sympathetic system.

The data gathered did not support the hypothesis stated above, as the HR of the isolated chick hearts increased when exposed to this drug in increasing concentrations. Indeed, it appears that in the absence of innervation PSE, still worked directly, i.e., by binding to adrenergic receptors on the embryonic isolated chicken heart. As for the whole chick embryo, the experimental results could not differentiate an indirect effect from a direct effect, since there are both possibilities that PSE 
worked to increase HR by binding directly to adrenergic receptors or via stimulation of sympathetic nerve terminals. Therefore, for future research, whole embryos will be treated with 6-OHDA for the destruction of the sympathetic nerve terminals in the 6-day whole embryo to rule out indirect effects. Another factor that could have been involved in the results obtained in this study may be the developing heart system itself. PSE's effects on the developing 4-chambered heart may depend on the expression of adrenergic receptors and/or sympathetic nerve development.

PSE is an over-the-counter (OTC) medication commonly sold under the brand name Sudafed ${ }^{\circledR}$. It is used to temporarily relieve nasal congestion due to the common cold, hay fever or other upper respiratory allergies, and sinus congestion and pressure. It promotes nasal and/or sinus drainage, and works as a decongestant by shrinking blood vessels in the nasal passages. In terms of safety, this drug is of particular concern for pregnant women and young children. Recommendations by its manufacturer, Sudafed ${ }^{\oplus}$, state that it should not be given to children under 4 years old or for longer than 7 days in a row. This drug is an FDA pregnancy category $\mathrm{C}$, which states animal reproduction studies have shown an adverse effect on the fetus and there are no adequate and well-controlled studies in humans. ${ }^{10}$ Adverse reaction data from human investigational studies and case studies suggest an evidentiary 4.2-fold increase risk of gastroschisis, a defect in the anterior abdominal wall through which the abdominal contents freely protrude in the first trimester with use of the decongestant." Sympathomimetic drugs mimic the effects of neurotransmitter substances of the sympathetic nervous system, therefore drugs such as pseudoephedrine may activate $\alpha$-adrenergic receptors causing vasoconstriction in the uterine arteries, which may affect the blood flow to the fetus. This process could explain the association between the use of pseudoephedrine in the first trimester and the development of gastroschisis. Evidence proposes that this effect is negligible at recommended dosages; therefore, the drug may be given if the benefits justify the potential risk to the fetus. ${ }^{9}$ Based on experimental data gathered in this study on developing chick hearts and from the FDA Classification of Drug Safety During Pregnancy list, it appears that there is evidence of possible fetal risk. When pregnant women is taking Sudafed the baby also is exposed, for all substances cross the placenta through the umbilical cord and enter into the baby's bloodstream. Once in the embryo's bloodstream the drug may work directly and/or indirectly to increase HR in the fetus. When taken in high doses, it may lead to many arrhythmias and further complications to the embryo. The indistinguishable effect of the drug as seen in the study is significant because Sudafed ${ }^{\circ}$ is a commonly used decongestant. Without thorough understanding of the effects of the drug, it is difficult to ensure its safe use by the public. It is also important to reiterate that little research has been done on a vertebrate embryo. Therefore, further research is warranted to determine whether PSE will harm a developing human fetus in order to safeguard or prevent the use of the drug by pregnant women. This research shed some understanding as it assessed the effects of PSE on a developing heart system in various concentrations; studying dose response is central to determining "safe" and "hazardous" levels and dosages for drug. The data enclosed in this paper supports the finding of dangerous arrhythmias at elevated doses, while at low concentrations the isolated hearts and whole embryos illustrated a normal sinus rhythm. These observations suggest that the risks can be reduced if Sudafed is used as directed on the label, and not used in larger amounts.

Additionally, it is important to note that PSE is a key ingredient used to illegally produce the drug methamphetamine or "crystal meth" and is therefore kept behind the counters in local pharmacies in order to help keep drug abuse rates down. Pediatric pseudoephedrine use seems to be declining since the U.S. Congress passed the Combat Methamphetamine Epidemic Act of 2006. By law, products containing pseudoephedrine must now be sold behind the pharmacy counter and through online retailers who must meet certain requirement ${ }^{12}$. Given the adverse effects of PSE at high dosages presented in this paper and its ability to be converted into methamphetamine, it is strongly suggested that this drug be further regulated by becoming a prescribed medication. By making PSE less accessible, it may reduce the risks associated with the abuse of this drug and benefit society.

Overall, this research utilized denervated chick hearts, intact whole embryos along with various 
PSE concentrations to understand the pharmacodynamics of PSE on a developing vertebrate heart. Pharmacodynamics is the study of the biochemical and physiologic effects of drugs, dose-dependent relationships, and their mechanisms of action (drug/receptor interaction). Data illustrated that this drug increased the HR via a direct and/or indirect mechanisms on adrenergic receptors. These effects were dose-dependent and ranged by varying levels of exposure as more adverse effects were observed towards high concentrations. Understanding pharmacodynamics is critical for the clinical development of new drugs. Knowledge of such biochemical pathways is critical to finding the no-effect dose and the maximal effective dose so that an optimal therapeutic dose range can be selected. This research demonstrated that even though PSE can change the heart rate and cause arrhythmias, it does have a safe dose range, therefore risks can be reduced if taken in recommended amounts. However, the established dose range is based on studies in fully developed heart systems. Therefore, further research of this drug's effect on a developing vertebrate heart may help establish a more specific and safer dose range for pregnant women.

\section{ACKNOWLEDGMENTS}

We would like express gratitude to Dr. Jacqueline McLaughlin for her continuous commitment to undergraduate research and dedication to mentorship. We would also like to recognize Melissa Coyle for her aid in the laboratory and Justin Muser for sharing his knowledge in statistical analysis.

\section{REFERENCES}

[1] Hamburger, V. \& Hamilton, H. L. (1951). A series of normal stages in the development of the chick embryo. Journal of Morphology, 88: 49-92.

[2] Hamburger, V. \& Hamilton, H. L. (1992). A series of normal stages in the development of the chick embryo. 1951. Developmental Dynamics, 195(4): 231-72

[3] Hill, M.A. (2014) Embryology Chicken Development. Retrieved July 30, 2014, from //php.med.unsw. edu.au/embryology/index.php?title=Chicken_Development.

[4] Reece, J.B., Campbell, N.A., Urry, L.A., et al. (2009). Campbell Biology, 9th edition. Boston: Pearson.

[5]Bektas, F., Eken, C. \& Okta, C. (2010). Pseudoephedrine-induced paranormal supraventricular tachycardia: a case report. The Journal of Emergency Medicine, 38: 53-57.

[6] Kobayashi, S., Endou, M., Sakuraya, F., et al. (2003). The sympathomimetic actions of I-ephedrine and d-pseudoephedrine: Direct receptor activation or norepinephrine release? International Anesthesia Research Society, 97: 1239-1245.

[7] Cruz, Y.P. (1993). Laboratory Exercises in Developmental Biology. Academic Press, San Diego, California, 241 pages. [ISBN 0-12-198390-0][book]

[8] McLaughlin, J. S., \& McCain, E. R. (1998). Developmental and physiological aspects of the chicken embryonic heart. Tested Studies for Laboratory Teaching, Volume 20. (C.A. Goldman, editor). Proceedings of the 20th Workshop/Conference of the Association for Biology Laboratory Education $(A B L E), 20: 85-100$.

[9] Wallace, Rosa. "Graphing Resources." Using Descriptive Statistics. National Science Foundation, 16 May 2005. Web. 6 Feb. 2015.

[10] Black, R.A. \& Hill, D.A. (2003). Over the counter medications in pregnancy. American Family Physician, 67: 2517-2524.

[11] Werler, M.M., Sheehan, J. E. \& Mitchell, A.A. (2002). Maternal medication use and risks of gastroschisis and small intestinal atresia. American Journal of Epidemiology, 155: 26-31.

[12] Vernacchio, L., Kelly, J.P., Kaufman, D.W., et al. (2008). Pseudoephedrine use among U.S. children, 1999-2006: results from the Slone Survey. Journal of the American Academy of Pediatrics, 122: 1299-1304. 


\section{ABOUT THE STUDENT AUTHORS}

Samely Gonzalez is a current undergraduate student at Penn State Abington, Philadelphia, PA set to graduate in May, 2016 with a B.S. degree in Genetics and Developmental Biology. She plans to utilize her skills and knowledge from undergraduate research and other science experiences in her pursuit to become a physician assistant. Her overall objective is to have a career in the medical field that allows her to help others achieve a healthy way of living.

Fatima Afzal is from Bethlehem, Pennsylvania and is currently enrolled as an undergraduate sophomore at Penn State Berks, Reading, PA. She has declared her major in biochemistry, pursing the cell and molecular biology option. She plans on applying the acquired research skills to future careers in pharmaceutical companies or as a medicinal chemist, working alongside eager scientists to develop and manufacture pharmaceuticals in order to grow and expand the drug industry.

\section{PRESS SUMMARY}

Pseudophedrine (PSE) is an over the counter (OTC) medication commonly used to temporarily relieve nasal congestion, upper respiratory allergies, and sinus pressure. Previous research has shown, however, that this drug elevates heart rate and blood pressure in humans and other mammals. This research utilized the chick embryonic heart as a model system to study the effects of (PSE) on the developing, vertebrate four-chambered heart and to determine whether this drug has either direct or indirect effects, i.e., it works by binding directly to receptors in the heart's pacemaker or indirectly by causing the release of norepinephrine from sympathetic nerve terminals to activate the same receptors. To do this, isolated developing chick hearts without innervation and whole chick embryos were exposed to varying PSE concentrations and heart rates were recorded. Research suggests that this drug has both direct and indirect effects and induces atrial flutter when exposed to high dosages. 


\title{
Nutritional Stress of Cultured Vero Cells Causes Altered Growth and Morphology as Seen in Neoplastic Transformation
}

\author{
Tyler Adams, Rabia Anwar, Michael Mfarej, Taylor Rundatz, \\ Melissa Coyle, and Dr. Jacqueline S. McLaughlin \\ Department of Biology, Penn State University Lehigh Valley, Center Valley, PA \\ Students: tadams1845@gmail.com,ranwar997@gmail.com,mfarejm@yahoo.com, \\ taylorrundatz4@gmail.com \\ Mentors: jxm57@psu.edu,msg110@psu.edu
}

\begin{abstract}
In this report, the authors describe the effects of stressed culture conditions on Vero cells, which are a form of epithelial cells derived from the African green monkey kidney. This project was designed in large part to replicate a previously published report on the effects of nutritional stress on the growth patterns of these cells. Culture conditions that include nutritional limitation and cell crowding have been shown to transform these cells into cells that differ morphologically, develop into spheroid-shaped clusters, as well as exhibit altered protein expression. It was suggested that these changes might mirror those occurring in metastatic cancer cells, and could therefore provide a useful experimental model system. In the current study, the investigators successfully cultured Vero cells in control cultures, and then compared their growth patterns and morphology to cells grown in nutritionally stressed and overcrowded cell culture conditions defined by extensive days in culture in unchanged media which did or did not contain glucose. The results confirmed that nutritionally stressed and over-crowded cultures result in cells that change morphologically, detach from the substrate, and exhibit spheroid-shaped clusters of cells. Sub-culturing these detached cells demonstrated that the results were permanent, meaning that the new growth patterns and clustering persisted. The results suggest that deprivation of nutrition and other factors essential to life may facilitate aberrant growth.
\end{abstract}

\section{KEYWORDS}

Vero Cells, Nutritional Stress, Cell Morphology, Neoplastic Transformation, Malignant Cells, Spheroid Formation.

\section{INTRODUCTION}

Vero cells are epithelial cells derived from the kidney of an African green monkey (Cercopithecus aethiops), which can be used for many purposes including: screening for the toxin of Escherichia coli; as host cells for growing specific viruses; or, as host cells for eukaryotic parasites. The Vero cell lineage was developed in 1962 by Yasumura and Kawakita at Chiba University in Chiba, Japan. Vero cells have a distinctive growth pattern in culture. The cells of this lineage grow in monolayers, are elongated in shape, and are similar to fibroblast cells with little cytoplasmic granulation. When a Vero cell population reaches cellular confluence, sub-culture should be carried out; otherwise, the cells degenerate or lift off the culture flask surface. ${ }^{1}$

Transformation is a multistep process that often culminates in the production of neoplastic (malignant) cells. ${ }^{2}$ Fibroblasts and epithelial cells have been shown to undergo neoplastic transformation in cell culture. According to Smets, cellular transformation itself involves a modification or serial modifications in the cellular genome, which lead to an overall altered phenotype. ${ }^{3}$ Morphological changes can be seen through altered growth and adhesion and spreading characteristics. ${ }^{4-6}$ Importantly, cellular transformation can be induced in vitro by several factors such as viruses, 
chemicals, radiation and metabolic injury, and as such, transformed cells have been used to study processes comparable to neoplastic transformation in vivo to better understand the origin and progression of tumors in animals. ${ }^{7-9}$ The interest here focuses on cellular transformation of Vero cells that is induced by nutritional stress and over-crowding.

Vero cells have also been shown to be a model system for studying nutritional stress-induced cellular transformation in vitro. If grown to confluence and left without media change for up to 15 days, nutritionally stressed Vero cells exhibit multilayered growth with many cells either detaching and growing freely in suspension or aggregating into cellular clusters called "spheroids". ${ }^{10}$ Normal fibroblasts or epithelial cells require adhesion and spreading on the substrate for growth in vitro, while several tumor cell lines present the ability to grow in suspension. ${ }^{11}$ Spheroids have been extensively characterized in cancer cells and embryonic cells ${ }^{12-14}$ and numerous research gives evidence that malignant cells of solid tumors, fetal cells of various organs, and liver cells of newborn rats aggregate and form spheroids when they are cultured on non-adhesive substrata. ${ }^{13}$ Additionally, cells participating in spheroid formation exhibit decreased glucose consumption, lactic acid production, and ATP content compared to cells in a monolayer, i.e., anaerobic metabolism ${ }^{14}$ and reveal higher polyploidy index than non-spheroidal cells. ${ }^{1}$

The goal of this experiment was to provide further evidence that Vero cell transformation is inducible in cell culture by engineering culture conditions with, and without, nutritional stress and overcrowding. Vero cells were grown over both nine day and fifteen day periods under the following conditions: control group with glucose and media change; experimental group with glucose and no media change; and, experimental group without glucose and no media change. Cell growth patterns and morphology were extensively monitored over this time period on a daily basis. For the fifteen day study, supernatant, which included spontaneously detached cells and spheroids, was collected and placed in culture flasks for an additional seven day period. The overall results substantiate that cell starvation and over-crowding in culture results in cells that detach from the substrate and/or exhibit spheroid-shaped clusters of cells. Additionally, the sub-culturing of these cells demonstrated that these morphological changes and behaviors are permanent, meaning that free-floating cells and spheroidal clusters persisted and were viable.

\section{METHODS AND MATERIALS}

\section{A. Control Culture Conditions}

Vero cells, obtained from Penn State York campus, were maintained in Roswell Park Memorial Institute (RPMI) 1640 media containing $2 \mathrm{~g} / \mathrm{L}$ glucose (Cellgro, 10-040-CM) supplemented with 5\% fetal bovine serum (FBS; Cellgro, 35-010-CV) and 1\% penicillin-streptomycin (Hyclone, SV30010). The cells were stored at $37^{\circ} \mathrm{C}$ with $5 \% \mathrm{CO}_{2}$ (ThermoForma Series II). The cells maintained under these conditions were known as the "control glucose" group (CG) since the media was replenished every 2-3 days. Sub-culture was not performed at confluence of the monolayers, and two groups of cultures were maintained for both nine and fifteen days (Figure 1). For the nine day experiment, ten T-25 flasks of cells were maintained for the CG group (Figure 1A) and were analyzed on a daily basis for cell confluency and morphology (Figure 1A). Importantly, one flask from this group was selected on each day of experimentation for analysis of cell count and viability. For the fifteen day experiment, fifteen T-25 flasks were maintained for the CG group and flasks were analyzed on a daily basis for cell confluency and morphology. Cell counts and viability were obtained from CG flasks on day 15 only, and both the adhered cells and culture supernatant were analyzed (Figure 1B).

\section{B. Experimental Culture Conditions}

Two nutritionally stressed and overcrowded experimental groups were created: "experimental glucose" (EG) and "experimental no glucose" (ENG). Both groups were maintained without change of culture media for both nine and fifteen days. These time spans were chosen as changes in growth 
characteristics were not observed over shorter periods of time, and most cell cultures presented senescence or death in cultures over 15 days - as per the work of Genari et al. (1998). ${ }^{10}$ EG cells were grown to confluence in RPMI 1640 containing $2 \mathrm{~g} / \mathrm{L}$ glucose (Cellgro, 10-040-CV) supplemented with 5\% FBS (Cellgro, 35-010-CV) and 1\% penicillin-streptomycin (Hyclone, SV30010) in $37^{\circ} \mathrm{C}$ with $5 \% \mathrm{CO}_{2}$. ENG cells were maintained in the same growth media, RPMI 1640, but contained no glucose (Cellgro, 10-043-CV) supplemented with 5\% FBS (Cellgro, 35-010-CV) and 1\% penicillin-streptomycin (Hyclone, SV30010). For the nine day experiment, ten T-25 flasks of cells were maintained for each of the EG and ENG groups (Figure 1A) and were analyzed for confluency and morphology on a daily basis. Importantly, one flask from this group was selected on each day of experimentation for analysis of cell count and viability. For the fifteen day experiment, fifteen T-25 flasks were maintained for each of the EG and ENG groups and were analyzed on a daily basis for cell confluency and morphology. Importantly, cell counts and viability were obtained from EG and ENG flasks on day 15 only, and both adhered cells and culture supernatant were analyzed (Figure 1B).

A.

\begin{tabular}{|c|c|c|c|c|c|c|c|c|c|}
\hline \multirow[b]{3}{*}{ CG } & & & & & Days & & & & \\
\hline & 1 & 2 & 3 & 4 & $\overline{55}$ & 6 & 7 & 8 & 9 \\
\hline & & & & & & & & & \\
\hline EG & & & & & & & & & \\
\hline ENG & & & & & & & & & \\
\hline
\end{tabular}

\begin{tabular}{|c|c|c|c|c|c|c|c|c|c|c|c|c|c|c|c|}
\hline \multicolumn{7}{|l|}{ B. } & \multicolumn{2}{|c|}{ Days } & & & & & & & \\
\hline 1 & 2 & 3 & 4 & 5 & 6 & 7 & 8 & 9 & 10 & 11 & 12 & 13 & 14 & 15 & \\
\hline CG & & & & & & & & & & & & & & & \\
\hline EG & & & & & & & & & & & & & & & 7 Dav subculture \\
\hline & & & & & & & & & & & & & & & \\
\hline
\end{tabular}

Figure 1. Experimental design showing culture conditions for the 9 day preliminary study (A) and the subsequent 15 study (B). Media change is denoted by blue boxes in control groups (CG). Attached cells (adhered to flask) and suspended cells (free-floating in supernatant) were counted on day 15 and are represented by red boxes in CG, EG, and ENG groups. Following cell counting and viability analysis, the remaining supernatant was centrifuged, re-suspended in fresh media, re-plated, and monitored microscopically for an additional seven days. No media changes were performed on these additional seven days.

\section{Cell Plating}

On day 0 of both experiments, the CG, EG, and ENG cultures were prepared by transferring $5 \mathrm{~mL}$ of Vero cells at approximately $1 \times 10^{5}$ cells/mL into the above stated T-25 flasks containing respective media conditions.

\section{Confluency}

Once the cells were plated, cell confluency (percentage of cell culture space utilized by adhering cells) was monitored microscopically over either the nine or fifteen day experimental period. Confluency was determined by observing the proportion of the flask surface occupied by adhered cells.

\section{E. Photomicrography}

Throughout the experimental period photomicrographs were taken daily of all three groups, for both 9 or 15 day experiments, using an Olympus CKX41 inverted phase-contrast microscope. Photomicrographs were taken at magnifications of 40X, 100X and 200X to document changes in cell morphology. 


\section{F. Cell Counting and Viability}

For the nine day experiment, trypsinization was performed daily on one flask in order to count attached cells using a hemocytometer. For the fifteen day experiment, trypsinization was performed on day 15 flasks only in order to count attached cells using a hemocytometer. Additionally, $1 \mathrm{~mL}$ of supernatant of cells from each group's labeled flasks was aliquoted into separate microcentrifuge tubes for cell counting. For both experiments, a trypan blue exclusion dye assay was also used to determine whether cells were viable. The trypan blue test was done by adding 50uL cell sample $+50 \mathrm{uL}$ trypan blue $+400 \mathrm{uL}$ PBS and mixing well. Next, 10uL of sample was loaded into each side of a hemocytometer slide.

\section{G. Supernatant and Subculture of Detached Cells}

Remaining supernatant from the day 15 CG, EG, and ENG group flasks was collected into 15 $\mathrm{mL}$ conical tubes. Each flask was then washed two times with $2 \mathrm{~mL}$ of D-PBS $\left(\mathrm{Ca}^{2+}\right.$ and $\mathrm{Mg}^{2+}$ free $)$ and added to its corresponding conical tube. The supernatant and D-PBS were then centrifuged for 5 minutes; the resulting cell pellet was re-suspended in $5 \mathrm{~mL}$ of fresh corresponding media and was added to a new T-25 flask. Corresponding flasks were returned to the incubator for an additional seven days. This was done to monitor any reattachment and growth of aggregates and to continually inspect suspended cells.

\section{RESULTS}

A preliminary nine day study was performed using Vero cells under the following conditions: as a control group with glucose and media change (CG); an experimental group with glucose and no media change (EG); and an experimental group without glucose and no media change (ENG) (Figure 1). It was reasoned that the CG group would grow to an over-crowded monolayer, while the EG and ENG groups would show stressed morphological changes due to nutritional stress and overcrowding. CG and EG groups reached peak confluency within 2-4 days after plating, while the ENG group never reached confluency. All groups showed no spheroid formation as nutritional deprivation and/or overcrowding increased over the nine day period; but, the ENG cells did become thin, elongated and branch-like and were less confluent (Figures $\mathbf{2}$ and $\mathbf{3}$; see Figure $\mathbf{5}$ for images of normal CG cell growth). Additionally, by day 8 of this preliminary study, ENG cells began to markedly detach from the flask surface (Figure 4A, examples shown with arrows) and exhibited noteworthy added stressed conditions of cytoplasmic vacuolation and granulation (Figure 4B, arrows). The latter morphological characteristics are abnormal in Vero cells and may indicate impaired cell health. ${ }^{15}$ This prompted a subsequent experiment employing a longer culture period of 15 days as evidenced in the work of Genari et al. (1998). ${ }^{10}$ It was reasoned, that by modifying the experimental conditions to include a 15 day culture period, cell detachment and spheroid formations, known to be associated with starvation and overcrowding, would be accentuated in the herein designed EG and ENG groups. 

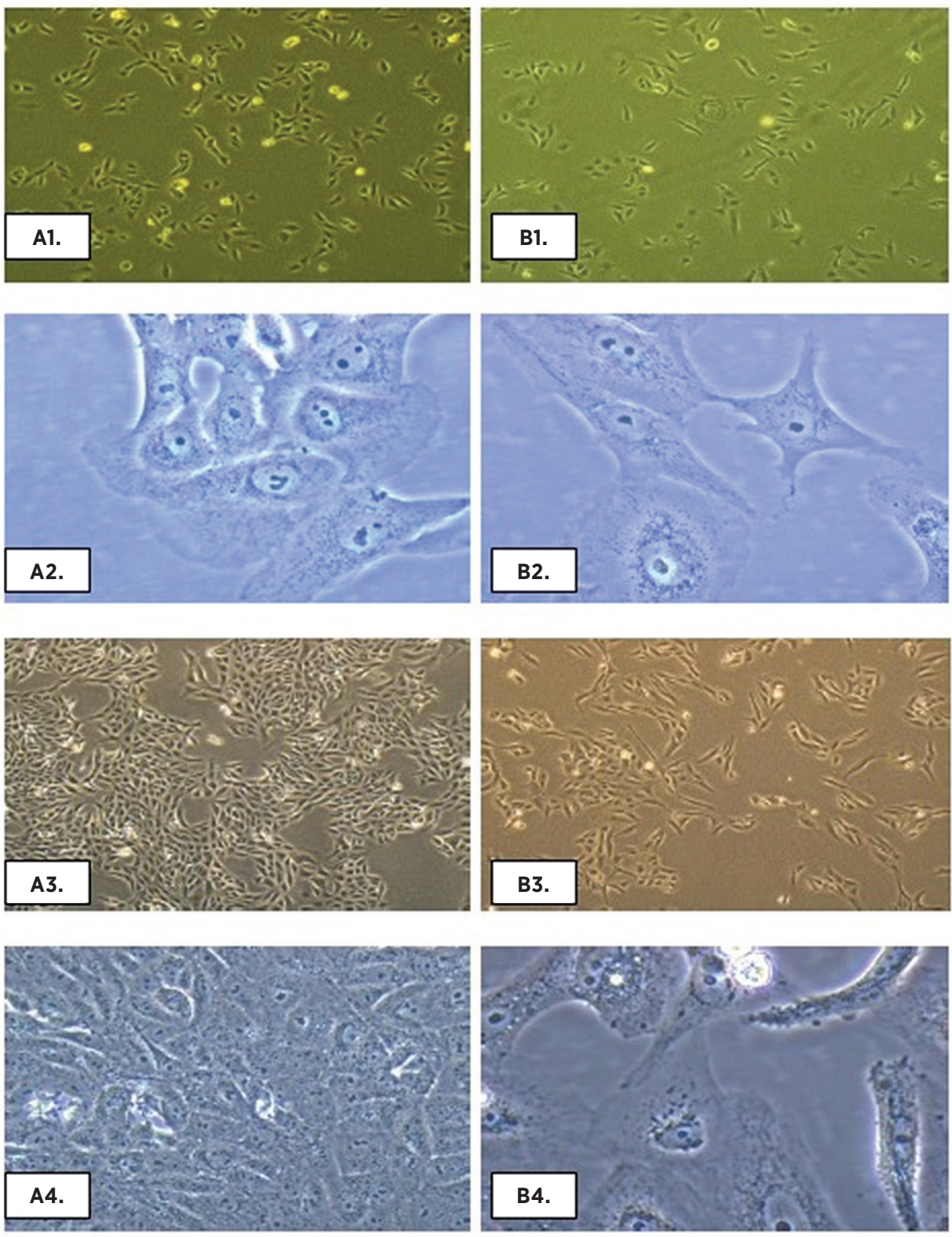

Figure 2. Photomicrographs contrasting the morphology of the EG cells. A. and the ENG cells B. from days 1-4 pilot study: A1 and B1 (40X); A2 and B2 (40X); A3 and B3 (40X); and, A4 and B4 (400X). Note elongated and thin shape in the ENG group and decreased cell confluency in comparison to EG group. 

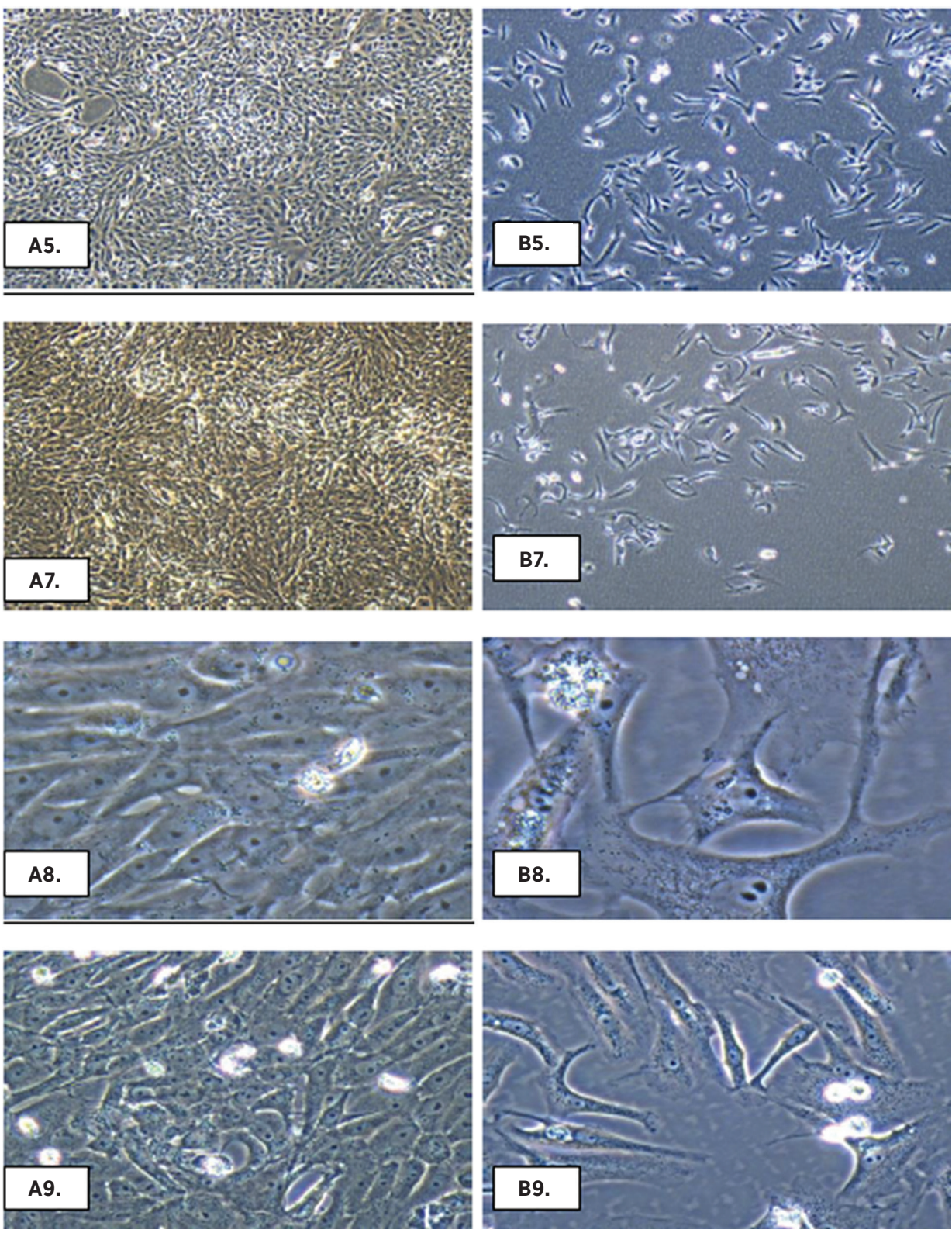

Figure 3. Photomicrographs contrasting the morphology of the EG cells (A) and the ENG cells (B) from days 5, 7, 8 and 9 pilot study: A5 and B5 (40X); A7 and B7 (40X); A8 and B8 (400X); and, A9 and B9 (200X). Note elongated and thin shape in the ENG group and decreased cell confluency in comparison to the EG group (A5-9). 


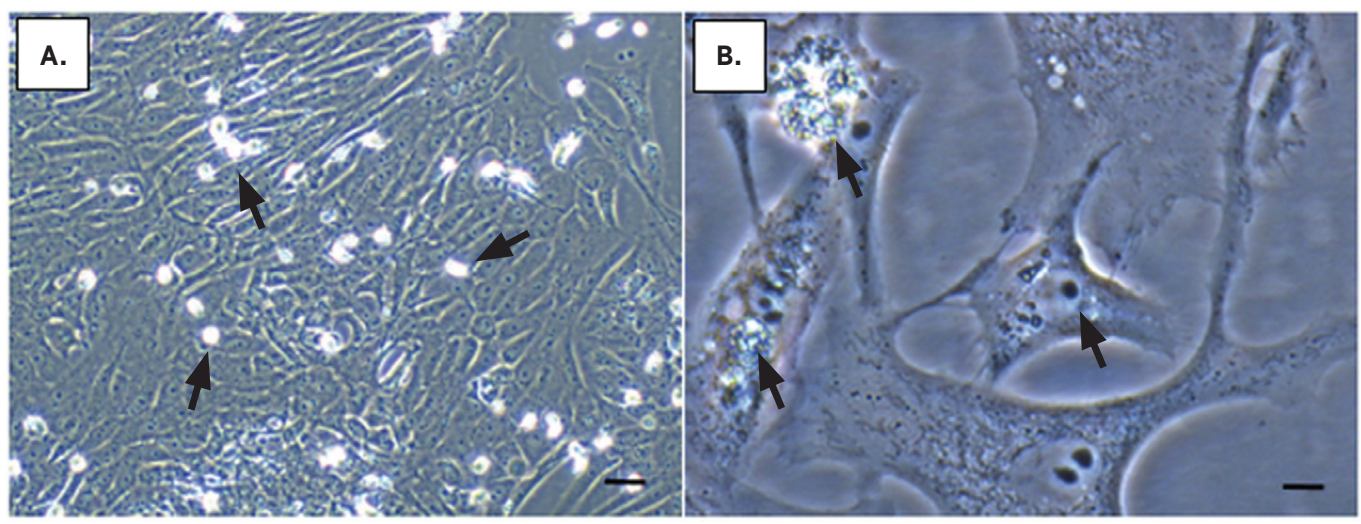

Figure 4. Images of Vero cell morphology in ENG conditions at day 8: (A) 400X (scale bar $=40 \mu \mathrm{m}$ ); and, (B) 400X (scale bar $=10 \mu \mathrm{m}$ ). Cells began to detach from the flask surface (Figure 4, arrows) and exhibited noteworthy added stressed conditions of cytoplasmic vacuolation and granulation (Figure 4B, arrows).

Similar to the pilot experiment, the CG and EG groups in the 15 day experiment reached peak confluency within 2-4 days after plating (Figure 5A-C). These two groups remained at peak confluency until days 8-9. At this time, however, the EG cells began to detach from the culture flask surface. Loss of surface adhesion was indicated by the appearance of cells that were more refractive and circular in shape as compared to adherent spindle-shaped cells. Concurrently, around day 8, the CG cells began to exhibit multi-layered growth which persisted until day 15 (Figure 5, C1 and D1). The EG cells did not exhibit the onset of multi-layered growth and remained as a confluent monolayer (Figure 5, C2 and D2). As for the ENG cells, as seen in the first experiment, confluence was never reached and cell detachment was more apparent beginning on days 8-9 and persisted until day 15 (Figure 5, C3 and D3).

As for spheroid formation, at days 13-15, EG cell growth behavior displayed the characteristic spheroid formation in culture (Figure 5, D3). Spheroidal aggregates appeared as multi-layered clusters, with non-adherent cells localized to the top surface. The cells at the peripheral edges of the spheroids were strictly elongated with diminished intercellular connections. The CG cells also displayed the formation of cell clusters at 13-15 days, but were much less concentrated and did not show the characteristics as described above. As for the ENG cell cultures on days 13-15, no spheroids were observed, and cell necrosis was apparent as cell confluence decreased and attached cells took on abnormal elongated appearances while detached cells increased in number. 


\section{Before Passage}

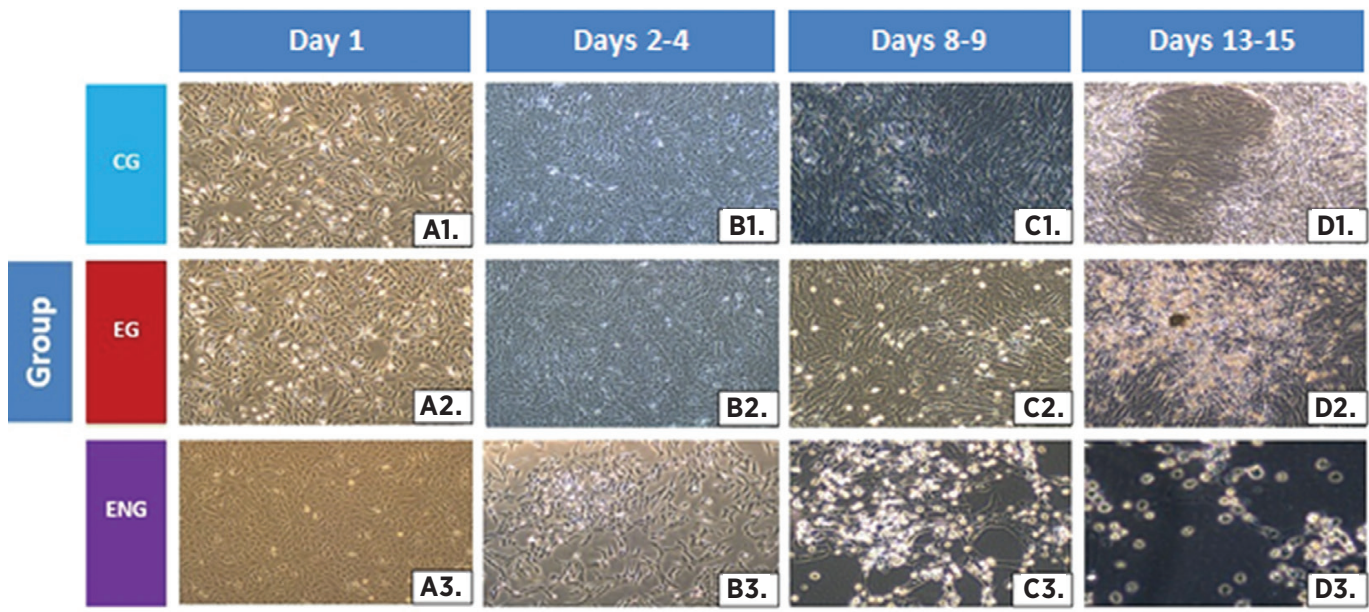

Figure 5. Photomicrographs of all groups at 40X assuming early adherence and normal morphology (A1 - A3). All groups at 40X displaying varying levels of confluency and substrate adherence (B1 - B3). All groups at 100X with EG and ENG cells exhibiting elongation and non-adherence (C1 - C3). CG and EG groups at 100X and ENG group at 200X with CG cells displaying multilayered overcrowding, EG cells with spheroid clusters, and ENG showing increased cell detachment and abnormal morphology (D1 - D3).

On day 15, cell counts and viability analysis of both the supernatant and the cells that remained attached to the flasks showed significant findings (Figure 6). The supernatant from the CG group contained very few detached cells (average $3.75 \times 10^{4}$ cells $/ \mathrm{mL}$ ); however, none of the detached cells were viable. The majority of the CG cells remained attached to the flask (average $8.05 \times 10^{6}$ cells/ $\mathrm{mL}$ ) with $99 \%$ viability. In contrast, EG cells presented an increased number of cells in the supernatant (average 1.50x $10^{5}$ cells $/ \mathrm{mL}$ ) with $25 \%$ of these detached cells being viable. This is essential to point out as this is the only group that presented a live detached cell population at an average concentration of $3.65 \times 10^{4}$ cells $/ \mathrm{mL}$. The portion of the EG cells which remained attached to the flask (average $6.88 \times 10^{5}$ cells $/ \mathrm{mL}$ ) were $87 \%$ viable. Lastly, while the supernatant of the ENG group confirmed observations of increased levels of cell detachment, this group also revealed the highest total cell concentration (average $2.63 \times 10^{5}$ cells $/ \mathrm{mL}$ ) in the supernatant with $0 \%$ viability. Notably, almost no cells remained attached to the flask in the ENG group (average $1.25 \times 10^{4} \mathrm{cells} / \mathrm{mL}$ ).

Since the EG group was the only group that presented live detached cells in the supernatant on day 15 , it was re-plated with fresh media and was monitored for an additional seven days. Figure 7 displays the EG group during the seven day period after passage. Within 24 hours after re-plating, the cells resumed spheroid formation (Figure 7A). Additionally, attached cells retained cytoplasmic granulation which was first evident before passage (Figure 7B). Other morphological changes noted included enhanced elongation and spindle-like cytoplasmic extensions. Moreover, seven days after sub-culturing, the cells showed aberrant plasmolytic (shrinkage or contraction of the protoplasm away from the wall of a living cell) morphology. The cells demonstrated failure to reestablish intercellular associations and normal spreading characteristics (Figure 7C). In summary, abnormal morphology and growth was maintained throughout the seven days after passage, i.e., cells did not recover normal Vero cell phenotype or monolayer growth pattern. 


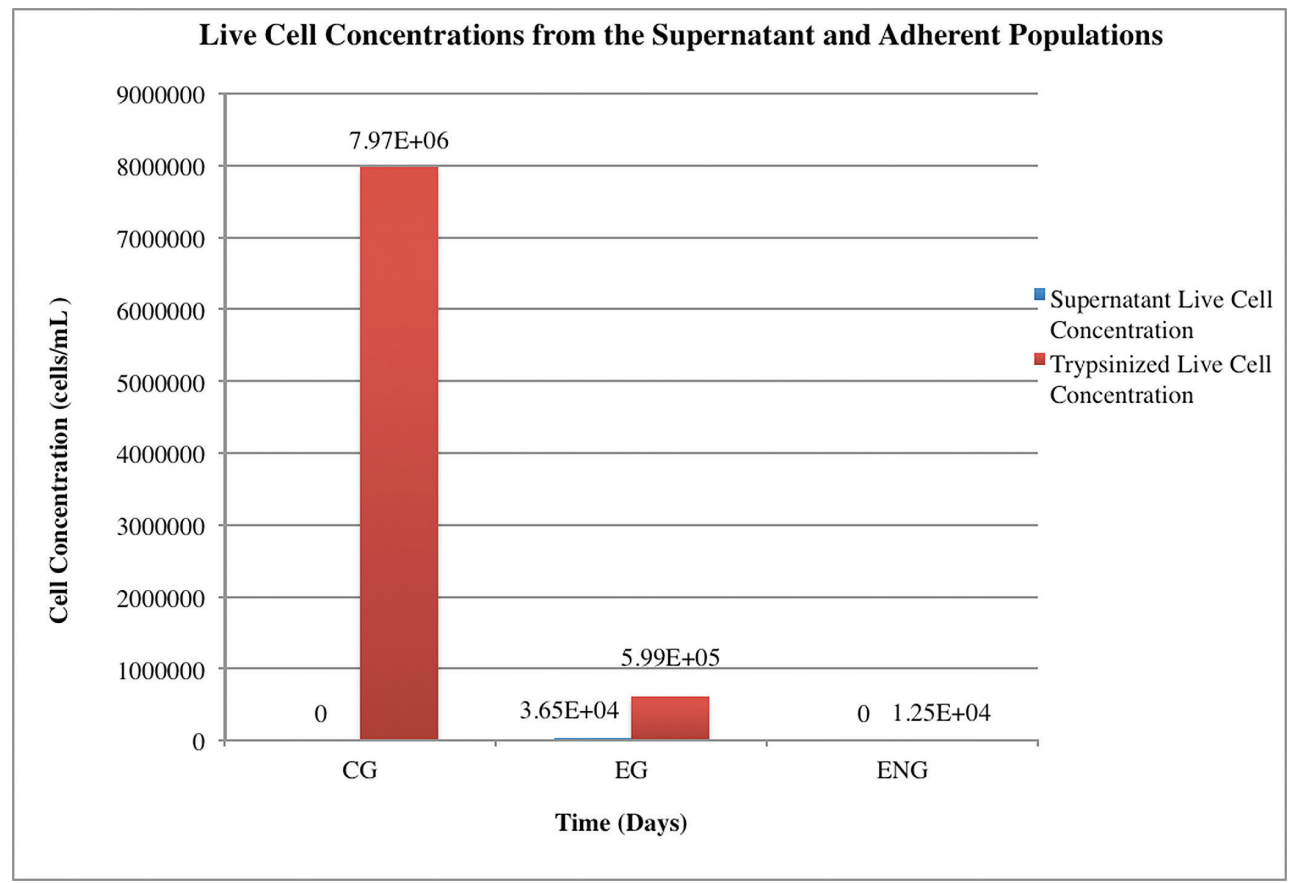

Figure 6. Histogram displaying live average cell count data taken on day 15 from the supernatant of the CG, EG, and ENG cultures (blue bars) and their trypsinized adherent cell fractions (red bars).
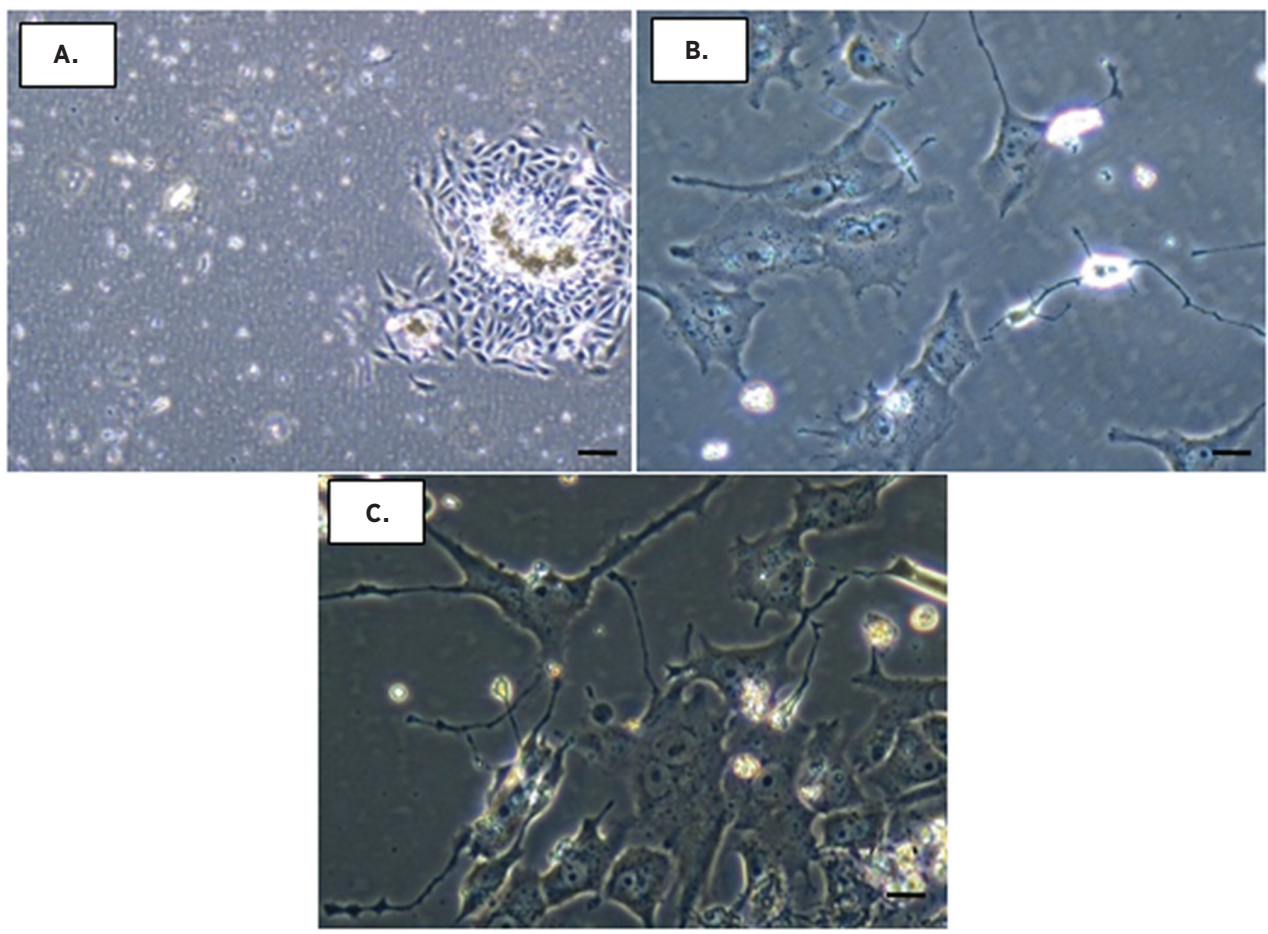

Figure 7. EG group displaying progression of abnormal cell morphology and growth characteristics after passage: A. $48 \mathrm{~h}$ (2 days) after passage showing re-adherence of transformed cells and spheroid formation (scale bar $=100 \mu \mathrm{m}$ ).

B. $144 \mathrm{~h}$ (6 days) after passage showing development of spindle-like cytoplasmic extensions (scale bar $=20 \mu \mathrm{m}$ ).

C. $168 \mathrm{~h}$ (7 days) after passage revealing prominent plasmolytic shape and reduced cell spreading (scale bar $=20 \mu \mathrm{m})$. 


\section{DISCUSSION}

The culture conditions employed in this experiment were used to further investigate previous findings on nutritionally-stressed and over-crowded Vero cells in vitro. Our results were similar, as the Vero cells grown without media change in the presence of normal media, the EG group, spontaneously detached after maintenance at peak confluency, being markedly noticeable at day 8 . This spontaneous detachment was accompanied by the formation of spheroid-shaped cell clusters between days 13-15. These cell aggregates formed in multiple layers, with detached cells on the top surface and attached cells at the peripheral edges being elongated with diminished intercellular connections. Also, the remaining attached cells developed marked cytoplasmic granules and vacuolization. These behaviors are notable deviations from normal Vero cell growth with media change (CG group herein), as this cell type typically grows as confluent monolayers and does not normally exhibit intracellular granulation or vacuolization. The observation that the Vero cells lost substrate adherence, however, is not a unique growth characteristic of this cells type or other cultured epithelial cell types. Substrate adhesion is facilitated by the establishment of critical components of the extracellular matrix (ECM). Loss of substrate adherence may be due to aberrant ECM synthesis which is an indication of enervated cell health. ${ }^{10,15}$ The additional finding that the EG group was the only group to possess viable detached cells in its supernatant at day 15 , however, does lend itself to support the postulate that nutritional stress and overcrowding were potential factors in the cell detachment of this group.

It is apparent that Vero cells grown in the presence of media without glucose and without media change, the ENG group, is lethal by 15 days. It is unclear as to why the cells deprived of glucose exhibit an elongated and abnormal shape change by day 8 in culture, but it is speculated that these cells are stretching to create cell to cell connections due to their less confluent nature. Indeed, as the confluency decreased the stretching of the cells became more evident, and this group never gained complete confluency during its nine days of culture.

Altered cell morphology and growth characteristics of the EG cells persisted after re-plating of the detached, floating cells and spheroid clusters that were centrifuged and re-suspended into fresh media at day 15. Moreover, abnormal EG cell morphology progressed further after re-plating. Augmented stress-induced morphological changes included spindle-like cytoplasmic extensions, degenerated intercellular associations, and plasmolytic shape. Additionally, these cells developed enhanced cytoplasmic granulation and vacuolization as compared to observations made before passage.

As previously noted, conditions of nutritional stress in Vero cells have been previously shown to induce deviant growth characteristics. ${ }^{10}$ Detachment of cells and spheroid formation has been explained as a loss of contact inhibition. This may also explain why the EG cells detached from their cell culture monolayer and were viable, while few control CG cells detached and those that did were non-viable. Prior work has documented successful culturing of nutritionally stressed detached Vero cells in soft agar medium. Such behavior has been interpreted as "anchorage independence," a characteristic which is deviant from standard Vero cell growth and commonly seen in malignancy. ${ }^{10,15}$

While loss of contact inhibition has been identified as a plausible underlying cellular process which promoted cell detachment and spheroid formation, other plausible processes include alterations in gene expression and regulation, cytoskeletal dynamics, extracellular matrix deposition and integrity, and/or second messenger systems - all of which are yet to be fully elucidated. Noteworthy is the work of Smets (1980) who associated cellular transformation with serial modifications of the cellular genome, resulting in irregular gene expression. ${ }^{3}$ Specifically, Genari et al. (1998) identified $44 \mathrm{kDa}$ and $78 \mathrm{kDa}$ proteins in nutritionally-stressed Vero cells as potential heat shock proteins (HSPs). ${ }^{15}$ The role of these two suspected HSPs is speculated to aid in sustaining the altered Vero cell phenotype. This was observed in conjunction with aberrant fibronectin expression in Vero cells that underwent cell transformation under nutritional stress conditions. ${ }^{10}$ Fibronectin 
is an essential component of the ECM. Previous work by Chakrabarty et al. (1997) explained that the ECM modulates cellular processes such as cell proliferation and gene expression. ${ }^{16}$ Mechanical stimuli transmitted through the ECM can mobilize intracellular signal cascades to affect gene expression..$^{17}$ Indeed, abrogated fibronectin is a trademark of epithelial tumor development. ${ }^{16}$ Interestingly, transformed chick embryo fibroblasts have been used as a model for the return of normal morphology, surface adhesion, and contact inhibition when the expression of major glycoproteins are restored. ${ }^{18}$ This further demonstrates the importance of the extracellular matrix in normal mammalian cell growth and the potential effects of cellular crowding on altering gene expression.

In summary, the results presented here confirm the essential roles that media change and sub-culturing procedures play in maintaining the morphological integrity of Vero cells, as shown previously by Genari et al. (1998)..$^{10}$ These results also demonstrate the utility of Vero cells as a model to study cell transformation induced by artificially implemented nutritional stress paradigms in vitro. As such, subsequent undergraduate research experiments that employ the Vero cell model can be used to further investigate the cumulative culture conditions that induced the changes observed in this paper. Before and after the fifteen day culture period, $\mathrm{pH}$, osmolarity, and glucose concentration measurements of the all culture conditions must be explicitly determined and compared, especially to those of the control group. This would provide insight into which stressors are most important in the transformation process. Indeed, little is known on the effect of glucose deprivation in transforming cell morphology. Some other unanswered questions include: What metabolic fuel source is relied upon by nutritionally-stressed and overcrowded Vero cells; How does ECM synthesis change as cells transition in detached and/or spheroid clusters; Are the transformation-like Vero cell morphology and growth changes truly analogous to neoplastic transformation processes at the genomic level, and if so in what ways? Subsequent experiments will also include other mammalian, adherent cell lines to determine if similar results can be generated in different cell types.

\section{ACKNOWLEDGMENTS}

We thank Penn State Lehigh Valley and Olympus for resource provisions. 


\section{REFERENCES}

[1] Genari, S., and Wada, M. (1995) Behavioral differences and cytogenic analysis of a transformed cellular population derived from a Vero cell line, Cytobioas 81, 17-25.

[2] Alberts, B., Johnson, A., Lewis, J., Raff, M., Roberts, K., and Walter, P. (2008) Cancer, In Molecular Biology of the Cell, pp 1209-1215, Garland Science, New York, NY.

[3] Smets, L. (1980) Cell transformation as a model for tumor induction and neoplastic growth, Biochemica et Biophysica Acta 605, 93-111.

[4] Keski-Oja, J., Alitalo, K., Barlati, S., and Vaheri, A. (1985) Pericellular matrix changes in fibroblastic and epithelial cells induced by oncogenic transformation, In Theories and Models in Cell Transformation (Tumori, I. S., Ed.), pp 55-70, Academic Press, Genoa, Italy.

[5] Nermut, M. V., Eason, P., Hirst, E. M., and Kellie, S. (1991) Cell/substratum adhesions in RSV-transformed rat fibroblasts, Exp Cell Res 193, 382-397.

[6] Vasiliev, J. M. (1985) Spreading of non-transformed and transformed cells, Biochimica et biophysica acta 780, 21-65.

[7] Group, I. N. E. W. (1985) Cellular and molecular mechanisms of cell transformation and standardization of transformation assays of established cell lines for the prediction of carcinogenic chemicals; overview and recommended protocols, Cancer Research 45, 2395-2399.

[8] Pónten, J. (1976) The relationship between in vitro transformation and tumor formation in vivo, Biochemica et Biophysica Acta 458, 397-422.

[9] Terzaghi-Howe, M. (1993) Factors regulating the emergence of spontaneous and X-ray-induced variants in primary rat tracheal epithelial cell cultures, In vitro cellular \& developmental biology: journal of the Tissue Culture Association 29A, 120-126.

[10] Genari, S. C., Gomes, L., and Wada, M. L. (1998) Alterations in the growth and adhesion pattern of Vero cells induced by nutritional stress conditions, Cell biology international 22, 285-294.

[11] Berenblum, I., and Armuth, V. (1981) Two independent aspects of tumor promotion, Biochimica et biophysica acta 651, 51-63.

[12] Genari, S. C., Dolder, M. A., and Wada, M. L. (1996) Scanning and transmission electron microscopy of transformed Vero cells, with altered in vitro growth characteristics, Journal of submicroscopic cytology and pathology 28, 565-572.

[13] Landry, J., Bernier, D., Ouellet, C., Goyette, R., and Marceau, N. (1985) Spheroidal aggregate culture of rat liver cells: histotypic reorganization, biomatrix deposition, and maintenance of functional activities, J Cell Biol 101, 914-923.

[14] Takezawa, T., Mori, Y., Yonaha, T., and Yoshizato, K. (1993) Characterization of morphology and cellular metabolism during the spheroid formation by fibroblasts, Exp Cell Res 208, 430-441.

[15] Freshney, I. (2011) In Culture of Animal Cells: A Manual of Basic Technique and Specialized Applications Sixth Edition ed., John Wiley \& Sons, Inc., Hoboken, NJ.

[16] Chakrabarty, S., Reynolds, S., Wang, H., and Rajagopal, S. (1997) Action of polypeptide growth factors in colon cancer: Development of new therapeutic approaches, In Frontiers in Bioscience 2.

[17] Alberts, B., Bray, D., Hopkin, K., Johnson, A., Lewis, J., Raff, M., K, R., and Walter, P. (2010) Cellular Communities: Tissues, Stem Cells, and Cancer, In Essential Cell Biology, pp 696-697, Garland Science, New York, NY.

[18] Yamada, K. M., Yamada, S. S., and Pastan, I. (1976) Cell surface protein partially restores morphology, adhesiveness, and contact inhibition of movement to transformed fibroblasts, Proc Natl Acad Sci USA 73, 1217-1221. 


\section{PRESS SUMMARY}

Vero cells were monitored for nine and fifteen day periods under nutritional deprivation and over-crowding conditions. The stressed cells remained viable and underwent a transformation in growth patterns and morphology analogous to behavior seen other models of mammalian cell transformation. These alterations were maintained in the stressed cells after re-plating, suggesting that the transformation was permanent. The results suggest that deprivation of nutrition and other factors essential to life may facilitate aberrant growth.

\section{ABOUT THE STUDENT AUTHORS}

Tyler Adams is currently a third year student at Penn State Berks. He is pursuing a B.S. in Biology. After graduation, he intends on earning his M.D. and specializing in cardiology.

Rabia Anwar is a third year student at Penn State Berks. She is pursuing a B.S. in Genetics and Developmental Biology and a minor in Entrepreneurship and Innovation. After graduation, she intends on earning her M.D. and specializing in cardiology.

Michael Mfarej is currently a fourth year Penn State Berks student. He is pursuing a B.S. in Genetics and Developmental Biology. He will be graduating in the Spring of 2015. After graduation, he plans to pursue a Ph.D. in Cellular and Molecular Biology with interests of continuing his research training in cancer biology.

Taylor Rundatz is currently a fourth year Penn State Berks student. She intends on graduating in the Spring of 2015 with a B.S. in Biology. After graduation, she plans to pursue an M.D. and Ph.D. dual degree program. With her medical education she is interested in a career as a pathologist. 


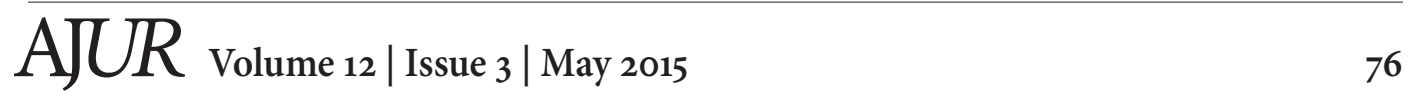




\title{
Searching for Gravitational Waves from the Coalescence of High-mass Black Hole Binaries
}

\author{
Liting Xiao ${ }^{a *}$, Alan J. Weinstein ${ }^{b}$, Dr. Tjonnie G. F. Li ${ }^{b}$, Surabhi Sachdev ${ }^{b}$ \\ ${ }^{a}$ Department of Physics, University of Virginia, Charlottesville, VA \\ ${ }^{b}$ LIGO Laboratory, California Institute of Technology, Pasadena, CA \\ *Student:lx8ad@virginia.edu \\ Mentors: ajw@ligo.caltech.edu,tgfli@ligo.caltech.edu,surabhi.sachdev@ligo.org
}

\begin{abstract}
We search for gravitational waves from the coalescence (inspiral, merger and ringdown) of binary black holes with data from the Laser Interferometer Gravitational-Wave Observatory (LIGO). Provided with well-described waveform models from General Relativity, matched filtering is employed in the GSTLAL analysis pipeline as the optimal detection technique for weak signals in Gaussian noise. The GSTLAL analysis pipeline filters data with waveform template banks, identifies triggers with SNR greater than 4, forms coincident triggers between multiple detectors in the LSC-Virgo Collaboration, and attempts to optimally separate signal from detector background noise fluctuations using a Chisquared test. We analyze high-statistics simulations of binary merger waveforms injected into LIGO recolored S6 data to evaluate the pipeline search sensitivity and to test the readiness of the pipeline for Advanced LIGO. With Advanced LIGO fully in operation by 2015 and the upgraded analysis pipelines, the expected detection rate is increased to as much as 100 events/year or more as compared to 0.01-1 events/year in Initial LIGO. Our work will make it possible to detect gravitational waves from binary black hole coalescence in Advanced LIGO data with high confidence.
\end{abstract}

\section{KEYWORDS}

LIGO, Gravitational Waves, General Relativity, Coalescence, Black Hole Binaries, Noise Fluctuations, Matched Filtering, Chi-squared Test, Simulations, GSTLAL Analysis Pipeline

\section{INTRODUCTION}

General Relativity predicts the presence of gravitational waves as the ripples of space-time curvature. Gravitational waves (GWs) propagate outwards at the speed of light from the sources of changing gravitational fields. As GWs pass by objects, the observer on earth would detect that the distances between objects increase and decrease periodically, known as the effects of strain. Astrophysical sources of GWs include compact binary coalescences (CBCs), pulsars, supernovae, gamma ray bursts, the Big Bang, etc. ${ }^{1}$ The amplitudes of GWs are estimated to be of the order of $10^{-21}$. $^{1}$ Direct detection of GWs has not been made yet. However, measurements of the Hulse-Taylor binary pulsar system provided indirect evidence of the existence of GWs. Orbital decay of the Hulse-Taylor system agrees with the prediction of General Relativity that the energy loss is due to emission of GWs. ${ }^{2}$

LIGO Scientific Collaboration (LSC) aims to directly detect gravitational waves using several Michelson interferometers located across the globe (Livingston in LA, Hanford in WA, GEO in Germany, and a planned LIGO in India) in association with Virgo in Italy and KAGRA in Japan. A schematic of the advanced LIGO interferometer is shown in Figure 1. Successful detection of GWs will provide a direct test of General Relativity and a probe into the most exotic, least understood objects in the universe, such as black holes, neutron stars, supernovae, and the Big Bang. ${ }^{1}$ The most 
promising source of ground-based detections is the CBCs, where the frequencies of GWs lie in the high frequency band (i.e. $10^{1}$ to $10^{4} \mathrm{~Hz}$ ) that LIGO is sensitive to. Initial LIGO has searched for CBCs (NS-NS, NS-BH, and BH-BH systems) as they spiral in towards each other and merge with an expected detection rate of 0.01-1 events/year from 2002 to 2012 but reported no unambiguous detections. ${ }^{3}$ Soon in 2015-2017, Advanced LIGO detectors will increase the amplitude sensitivity at least ten times, and will thereby increase the expected detection rate to as much as 100 events/ year or more. With the upgraded sensitivity, the probability of detecting CBCs, especially BH-BH systems, is greatly increased. ${ }^{4}$

The CBC-BBH working group in LIGO searches for GW signals coming from binary black hole (BBH) systems with the use of matched filtering detection technique. BBH systems involve two black holes orbiting around each other, radiating off GWs. As BBH systems give off GWs, they go through three phases of coalescence - inspiral, merger, and final ringdown (IMR). ${ }^{5}$ While for lowmass CBC systems only inspiral lies in the LIGO frequency band, all three coalescence phases are detectable in LIGO for high-mass BBH systems (each of mass greater than $M_{t o t}=\left(m_{1}+m_{2}\right)>10 M_{\odot}$ ).

Previously in Initial LIGO, the iHOPE analysis pipeline was implemented to search for non-spinning $\mathrm{BBH}$ systems, with lower mass region and higher mass region being searched separately. ${ }^{6}$ Nonetheless, BBH systems are predicted to contain substantial spins, either aligned spins from the death of high-mass stars, or precessing spins from dynamical captures. We hereby introduce the GSTLAL analysis pipeline that is currently under development to search for BBH systems with aligned spins (work to include precessing spins is underway), as well as a larger mass range. Unlike other pipelines in LIGO, the GSTLAL pipeline has low latency, which means it can initiate other astrophysical searches using electromagnetic telescopes immediately after flagging a detection in the pipeline. This is helpful in short gamma ray bursts and supernovae searches.

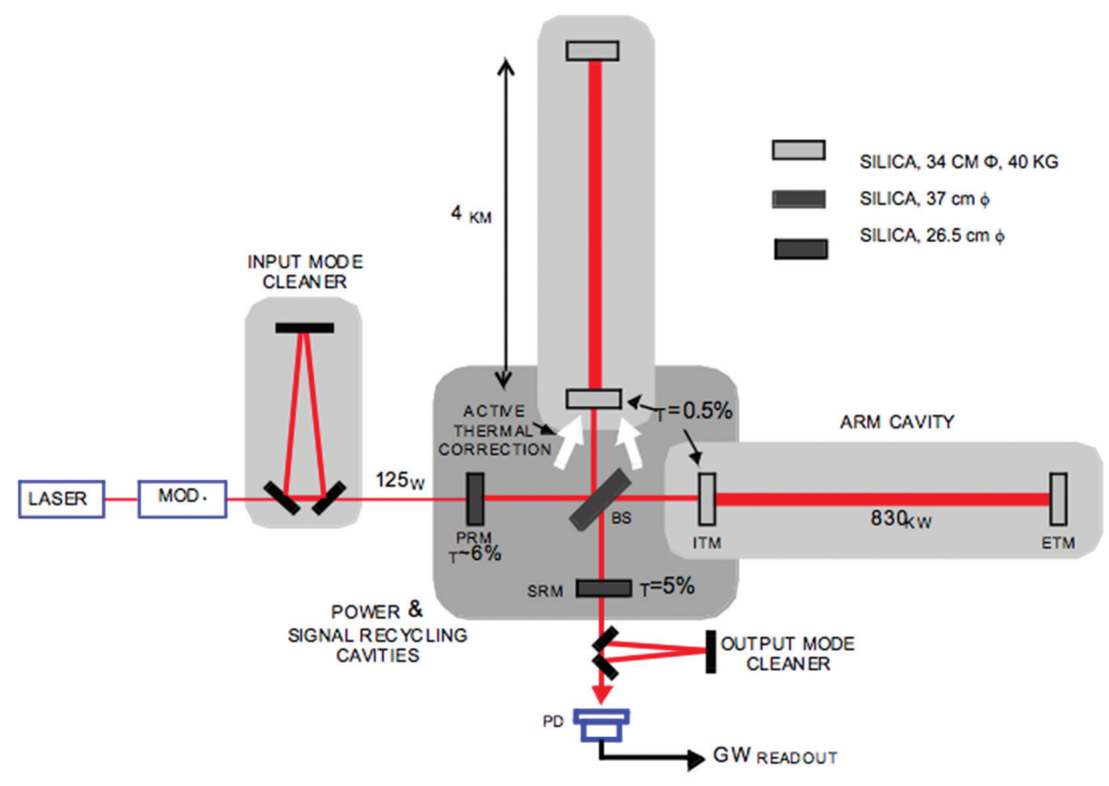

Figure 1. A schematic of the advanced LIGO interferometer. ETM = end test mass; ITM = input test mass; $\mathrm{PRM}=$ power recycling mirror; $\mathrm{SRM}=$ signal recycling mirror; $\mathrm{BS}=50 / 50$ beam splitter; $\mathrm{PD}=$ photodetector $^{\mathrm{MOD}}=$ phase modulation ${ }^{4}$ 


\section{THE GSTLAL ANALYSIS PIPELINE}

Gravitational waveforms from BBH systems are believed to be well described by General Relativity. Along with the simplification of the detector noise being stationary and Gaussian, matched filtering searches for GW signals are employed as the optimal detection technique. ${ }^{7}$ Matched filtering correlates a known signal, a template, with an unknown signal to detect the presence of the template in the data stream. In implementing matched filtering, however, the research problem is significantly complicated by the facts that (a) the exact parameters of the $\mathrm{BBH}$ system giving off the signal are not provided to us ahead of time, which requires the use of template banks to cover a large parameter space, and that (b) detector noise is by no means stationary and Gaussian, which is why we utilize a $\chi^{2}$ statistic to suppress the influence of non-Gaussian "glitches" in the data stream.

In the GSTLAL analysis pipeline implementation, we first construct stochastic banks of discretely sampled template waveforms within specified spin range and mass range. To make a stochastic bank, waveform parameters are proposed randomly and the template is admitted to the bank if satisfying certain criteria. Then data are matched filtered with the same template bank separately in different detectors, generating signal-to-noise ratio (SNR) for all possible filters/templates. Simultaneously, because of the non-Gaussian noise existing in GW detectors, a $\chi^{2}$ statistic is computed which compares the data to potential signal plus Gaussian noise. This is useful in distinguishing potential signals from triggers arising from non-Gaussian detector noise fluctuations, known as "glitches". Events exceeding a certain predetermined SNR cut (SNR $=4$ in the current case) are recorded as triggers of interest. Then triggers close in time are clustered together and only the one with the largest SNR is recorded. We then compare triggers from different detectors and look for coincident triggers within a certain tolerance in time and the exact match in masses and spins. Subsequently, a list of coincident triggers ranked by a likelihood ratio, computed from the SNR and $\chi^{2}$, is obtained. For the remaining coincident triggers, we further compute the false alarm probability (FAP) and the inverse false alarm rate (IFAR), to determine how likely it is to mistake noise as signals. We claim a detection if a trigger has an IFAR value larger than a threshold. ${ }^{9}$ A flowchart of the GSTLAL analysis pipeline is shown in Figure $\mathbf{2}$.

In this section, we discuss the waveform template banks and some important detection statistics, i.e., SNR, $\chi^{2}$, and FAP, in detailed derivations. 


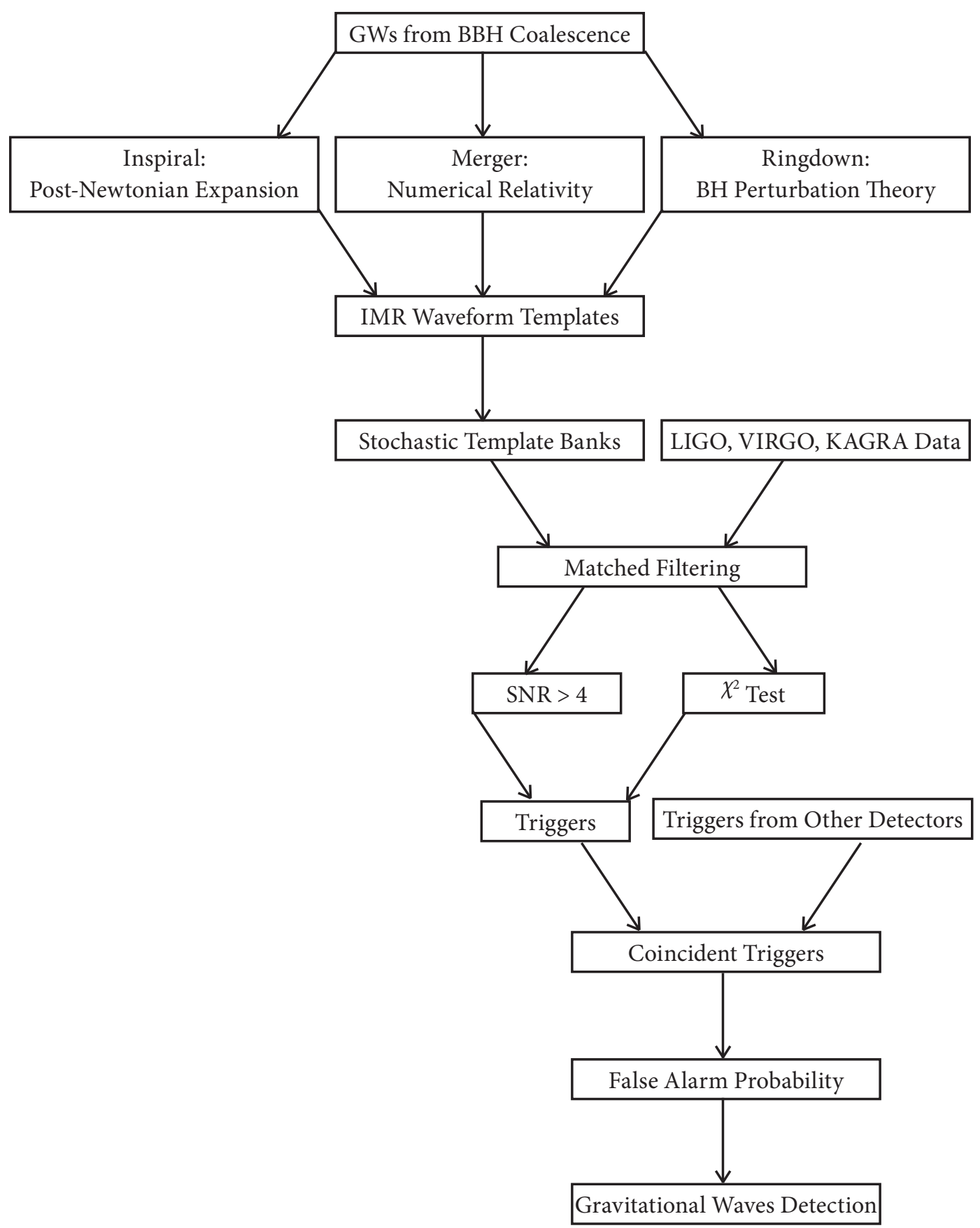

Figure 2. Flow chart of the GSTLAL analysis pipeline. 


\section{WAVEFORM TEMPLATE BANKS}

Since the parameters of BBH systems are unknown to us, we cover a large parameter space in the searches by constructing template banks. In GSTLAL, we make use of a phenomenological model, IMRPhenomB, to compute waveform templates that constitute template banks. Computing waveforms of $\mathrm{BBH}$ coalescence in different phases is done by different methods. ${ }^{9}$ The inspiral phase is well described by post-Newtonian expansion. The merger phase can be calculated through numerical relativity. The ringdown phase can be calculated by black-hole perturbation theory. ${ }^{5}$ Nonetheless, for a particular BBH system, 15 parameters are needed to specify the expected incoming waveform. Luckily, most extrinsic parameters only affect the overall amplitudes of signals, leaving us only intrinsic parameters to consider in order to cover the whole parameter space. These intrinsic parameters (not all independent to each other) include component masses $m_{1}, m_{2}$, the total mass of the system $M_{\text {tot }}=m_{1}+m_{2}$, mass ratio of the component masses $q=\max \left(m_{2} / m_{1}, m_{1} / m_{2}\right)$, and spins of the component masses in the binary system $\overrightarrow{S_{1}}, \overrightarrow{S_{2}}$.

IMRPhenomB incorporates all three (IMR) phases of the waveform, and includes the effect of aligned spin using a single parameter $\vec{\chi}_{\text {eff }}=\left(m_{1} \overrightarrow{S_{1}}+m_{2} \overrightarrow{S_{2}}\right)=M_{\text {tot }}$. Using this waveform model, the time domain and frequency domain waveforms for an example BBH system with $M_{1}=30 M_{\odot}$, $M_{2}=30 M_{\odot}$, distance $=100 \mathrm{Mpc}, s_{1 z}=0, s_{2 z}=0$ are shown in Figure 3. The three stages (i.e. inspiral, merger, ringdown) of coalescences are observable in both domains.

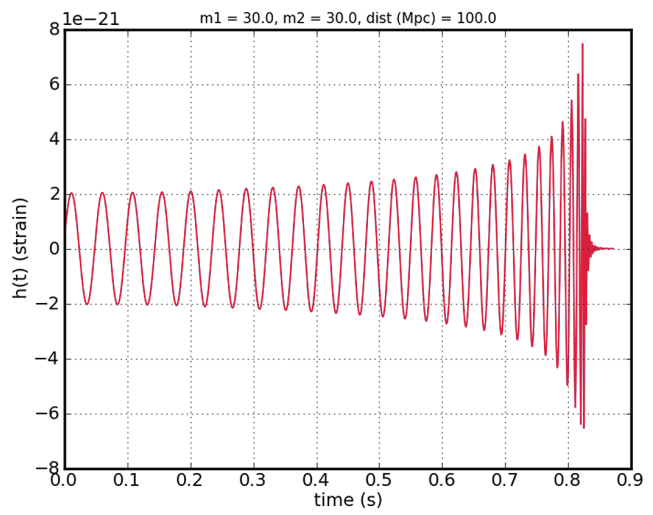

a. Time domain waveforms.

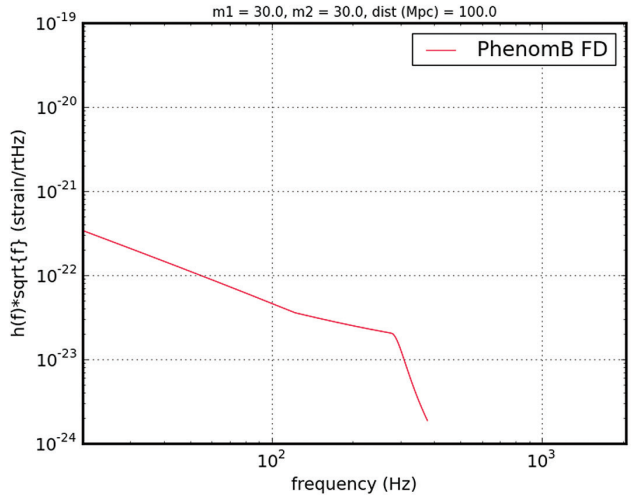

b. Frequency domain waveforms.

Figure 3. Example time domain and frequency domain waveforms of a BBH system. The frequency domain is the Fourier Transform of the time domain.

To generate template banks covering the parameter space (here, a 3-dimensional space comprising two component masses and one aligned spin parameter), lattice and stochastic template placement techniques could be used. In the GSTLAL pipeline implementation, stochastic banks are used. To generate a stochastic template bank, mass and spin parameters are proposed randomly first. If the proposed template's fitting factor, which describes how well a waveform "fits" another waveform, against the initial bank does not exceed a chosen minimum value (currently $F F_{\min }=0.97$ ), this template is accepted into the bank. Otherwise, the proposed template is discarded. This process is iterated until the rejection rate of proposed templates reaches a set value. $^{9}$ 


\section{SIGNAL-TO-NOISE RATIO}

The signal-to-noise ratio $\rho$ statistic used in the GSTLAL analysis pipeline, which characterizes the maximum detection probability for an event, is described as the optimal detection statistic for a single even. ${ }^{8}$

For a data set $s(t)=h(t)+n(t)$, where $h(t)$ is the signal and $n(t)$ is the Gaussian stationary noise, we define the SNR time series as

$$
\rho(t) \equiv \frac{|z(t)|}{\sigma},
$$

Equation 1.

where the cross-correlation of two waveforms is defined by their Hermitian inner product

$$
z(t)=\langle\widetilde{s} \mid \tilde{h}\rangle=4 \int_{0}^{\infty} \frac{\widetilde{s}(f) \tilde{h}^{*}(f)}{S_{n}(f)} e^{2 \pi i f t} d f,
$$

Equation 2.

and the measure of the sensitivity of detector $\sigma$ is

$$
\sigma=\sqrt{\langle\tilde{h} \mid \tilde{h}\rangle}=\sqrt{4 \int_{0}^{\infty} \frac{|\tilde{h}(f)|^{2}}{S_{n}(f)} d f .}
$$

Equation 3.

The tilde represents the Fourier Transform of the waveform, which is given by

$$
h(\tilde{f})=\int e^{-2 \pi i t} h(t) d t .
$$

Equation 4

And the noise power spectral density (PSD), which gives the average intensity at a given frequency, is defined as

$$
S_{n}(f)=2\left\langle|\tilde{n}(f)|^{2}\right\rangle,
$$

Equation 5.

where $\left\langle|\tilde{n}(f)|^{2}\right\rangle$ here denotes the average of $|\tilde{n}(f)|^{2}$.

A "trigger" is a peak exceeding 4 in the SNR time series, $\rho\left(t_{o}\right)>4$.

\section{$\chi^{2}$ TEST}

A $\chi^{2}$ test is used to determine whether the waveform template added to Gaussian noise is a good fit for the trigger." We compute an autocorrelation $\chi^{2}$ by comparing the SNR time series to the autocorrelation of the template.

In a data stream $s=A h+n, n$ is the detector noise and $A h$ is the signal, where $A$ is the amplitude of the signal and $\langle h, h\rangle=1$. We measure the SNR time series

$$
\rho(\tau)=\left\langle n, h e^{2 \pi i f \tau}\right\rangle+A\left\langle h, h e^{2 \pi i f \tau}\right\rangle
$$

Equation 6.

where $\tau$ is the time difference between the actual signal coalescence time and the time-shifted template. Since we do not know the BBH system's actual coalescence time, we define $\tau=0$ to be the time that gives the maximum SNR.

We define the autocorrelation of the whitened template as

$$
\alpha(\tau) \equiv\left\langle h, h e^{2 \pi i f \tau}\right\rangle .
$$

Equation 7.

If we maximize Equation 6 over time and take the ensemble average, the noise term will disappear, which then will give us $A \approx\left\langle\rho_{\max }\right\rangle$. And thus,

$$
\rho(\tau)-\left\langle\rho_{\max }\right\rangle \alpha(\tau)=\left\langle n, h e^{2 \pi i f \tau}\right\rangle .
$$

Equation 8. 
Quantities $\rho(\tau),\left\langle\rho_{\max }\right\rangle$, and $\alpha(\tau)$ are easily measurable from data and templates. We use these quantities to compute the $\chi^{2}$ statistic for an event,

$$
\chi^{2}=\int_{0}^{T_{\max }}\left|\rho(\tau)-\left\langle\rho_{\max }\right\rangle \alpha(\tau)\right|^{2} d \tau
$$

Equation 9.

If we only have Gaussian noise, SNR is the optimal detection statistic. However, with non-Gaussian detector noise, SNR statistic only is not good enough to separate signal and noise. Shown in Figure 4 is an example $\chi^{2}$ vs. SNR plot for triggers arising from simulated signals injected into recolored S6 data. We can see that some backgrounds also have high SNR values, and thus, without $\chi^{2}$ statistic, these backgrounds would blend into signals. Because noise triggers tend to have higher $\chi^{2}$ values than those from signal triggers, $\chi^{2}$ is very effective in differentiating signal from noise when non-Gaussian noise fluctuations are present.

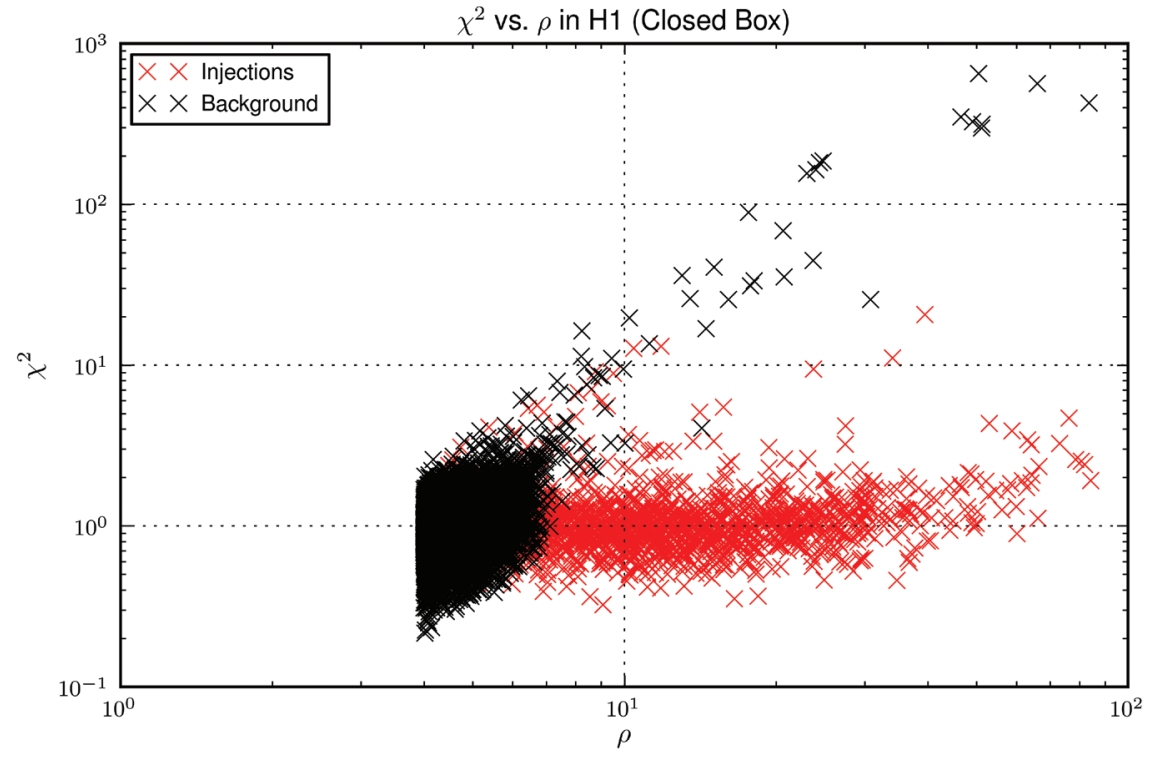

Figure 4. Example $\chi^{2}$ vs. SNR plot.

\section{FALSE ALARM PROBABILITY}

In the pipeline, it is also crucial to estimate the probability of observing a coincident event that is purely noise at the multi-detector level. False alarm probability $P(\mathcal{L} \mid n)$ is the probability of identifying noise as signal at a likelihood $\mathcal{L}$ when the data contain only noise. We aim to establish a FAP that does not depend on the time shifts between detectors, but depends on intrinsic parameters $\vec{\theta}$ (here, the template parameters $m_{1}, m_{2}$ and $\overrightarrow{\chi_{\text {eff }}}$ ) of BBH systems, matched filter SNR $\rho$, and $\chi^{2}$ statistic $^{10}$.

Assume that we have $N$ independent detectors. For each detector $i$, we rank the coincident events by standard likelihood ratio $\mathcal{L}$, which characterizes the likelihood of an event being signal as opposed to being noise,

$$
\mathcal{L}\left(\rho_{1}, \chi_{1}^{2}, \ldots \rho_{N}, \chi_{N}^{2}, \vec{\theta}\right)=\frac{P\left(\rho_{1}, \chi_{1}^{2}, \ldots \rho_{N}, \chi_{N}^{2}, \overrightarrow{\theta \dagger} s\right)}{P\left(\rho_{1}, \chi_{1}^{2}, \ldots \rho_{N}, \chi_{N}^{2}, \overrightarrow{\theta \dagger} n\right)},
$$

Equation 10.

where $P(\ldots \mid s)$ is the probability of observing the event when it is signal, and $P(\ldots \mid n)$ is the probability of observing the event when it is noise. 
Because the noise in the different detectors is independent, the total likelihood ratio is the product of likelihood ratios from the $N$ detectors,

$$
\left.\mathcal{L}\left(\rho_{1}, \chi_{1}^{2}, \ldots \rho_{N}, \chi_{N}^{2}, \vec{\theta}\right) \simeq \prod_{i=1}^{N} \mathcal{L}_{i} \rho_{i}, \chi_{i}^{2}, \vec{\theta}\right) .
$$

Equation 11.

The FAP is then, again due to the independence of different detectors,

$$
P(\mathcal{L}, \vec{\theta} \mid n)=\int_{\Sigma} P\left(\mathcal{L}_{1}, \ldots \mathcal{L}_{N}, \vec{\theta} \mid n\right) d^{N-1} \Sigma=\int_{\Sigma} \prod_{i=1}^{N} P\left(\mathcal{L}_{i}, \vec{\theta} \mid n\right) d^{N-1} \Sigma, \quad \text { Equation } 12
$$

where $\Sigma$ is the contour of constant likelihood $\mathcal{L}=\prod_{i=1}^{N} \mathcal{L}_{i}$. And $P\left(\mathcal{L}_{i}, \vec{\theta} \mid n\right)$ is the result of marginalization of $P\left(\rho_{i}, \chi_{i}^{2}, \vec{\theta} \mid n\right)$ over $\rho_{i}$ and $\chi_{i}^{2}$,

$$
P\left(\mathcal{L}_{i}, \vec{\theta} \mid n\right)=\int_{\delta} P\left(\rho_{i}, \chi_{i}^{2}, \vec{\theta} \mid n\right) d \delta, \quad \text { Equation } 13 .
$$

where $\delta$ is the contour of constant likelihood $\mathcal{L}_{i}$ in the space of $\left\{\rho_{i}, \chi_{i}^{2}\right\}$ at constant $\vec{\theta}$.

Finally, to determine a FAP over a broad range of parameter space $\vec{\theta}$, we average (marginalize) over $\vec{\theta}$ (the template parameters $m_{1}, m_{2}$ and $\overrightarrow{\chi_{\text {eff }}}$ ):

$$
P(\mathcal{L}, n)=\int P(\mathcal{L}, \vec{\theta} \mid n) d \vec{\theta}
$$

Equation 14.

In application, the FAP of observing one noise event having likelihood ratio larger than a threshold $\mathcal{L}^{*}$ is

$$
P\left(\mathcal{L} \geq \mathcal{L}^{*} \mid n\right)=\int_{\mathcal{L}^{*}}^{\infty} P(\mathcal{L} \mid n) d \mathcal{L} . \quad \text { Equation } 15 .
$$

Thus, by binomial expansion, the FAP of observing at least one noise event having likelihood ratio greater than a threshold $\mathcal{L}^{*}$ within a sample of $M$ coincident triggers is,

$$
\begin{aligned}
P\left(\mathcal{L} \geq \mathcal{L}^{*} \mid n_{1}, \ldots, n_{M}\right) & =1-\left(\begin{array}{c}
M \\
0
\end{array}\right) P\left(\mathcal{L} \geq \mathcal{L}^{*} \mid n\right)^{0}\left[1-P\left(\mathcal{L} \geq \mathcal{L}^{*} \mid n\right)\right]^{\mathrm{M}} \\
& =1-\left[1-P\left(\mathcal{L} \geq \mathcal{L}^{*} \mid n\right)\right]^{\mathrm{M}} .
\end{aligned}
$$

Equation 16.

For a noise event, it is expected that the noise event follows Poisson Statistics. ${ }^{10}$ Thus, the probability of observing one noise event with a Poisson mean $\lambda$ is defined as,

$$
P(N=1 \mid n)=1-e^{-\lambda} \sum_{j=0}^{N-1} \frac{\lambda^{j}}{j !} .
$$

Equation 17.

Combining Equation 16 and Equation 17, we obtain the mean number of noise events with likelihood ratio $\mathcal{L} \geq \mathcal{L}^{*}$,

$$
\lambda\left(\mathcal{L} \geq \mathcal{L}^{*}\right)=-\ln \left[1-P\left(\mathcal{L} \geq \mathcal{L}^{*}\right)\right] .
$$

Equation 18.

Substitute the mean $\lambda$ from Equation 18 back into Equation 17, we get the probability of observing $N$ noise events with likelihood ratio $\mathcal{L} \geq \mathcal{L}^{*}$,

$$
P(N \mid n)=1-e^{-\lambda\left(\mathcal{L} \geq \mathcal{L}^{*}\right)} \sum_{j=0}^{N-1} \frac{\lambda\left(\mathcal{L} \geq \mathcal{L}^{*}\right)^{j}}{j !} .
$$

Equation 19.

In practice, nevertheless, the pipeline computes the FAP from the observed distribution in likelihood $\mathcal{L}$ of triggers. The event likelihood is calculated by locating its $\rho, \chi^{2}$ values in a 2 -dimensional histogram for signal and for background; a typical background histogram is shown in Figure 5a. 
Figure 5a shows the likelihood ratio $\mathcal{L}_{H 1}$ as a function of $\operatorname{SNR} \rho$ and $\chi^{2}$ for detector H1. It is expected that a coincident event with higher SNR $\rho$ and lower $\chi^{2}$ would be ranked as having a higher likelihood of being a signal. Red here in the plot indicates higher likelihood ratio values. The resulting FAP distribution for candidate triggers falls off with increasing likelihood value shown in Figure 5b. Figure 5b shows the probability of capturing a noise event with a signal likelihood ratio $\mathcal{L}$ or greater. Again, as expected, as the likelihood ratio $\mathcal{L}$ increases, the probability FAP of events being noise goes down. The grey area suggests that the uncertainty in FAP calculation also increases with increasing likelihood.

Furthermore, traditionally a quantity derived from FAP is utilized to estimate the significance of coincident triggers, which is the inverse false alarm rate, defined using Equation $\mathbf{1 8}$ as,

$$
\operatorname{IFAR}=\frac{\mathrm{T}}{\lambda}
$$

Equation 20.

where $\mathrm{T}$ is the total run time of the observation.

Coincident triggers with large IFAR are our detection candidate events.

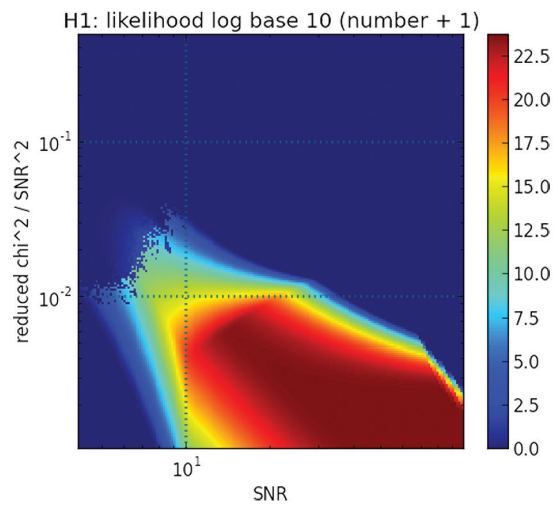

a. An example likelihood distribution in noise.

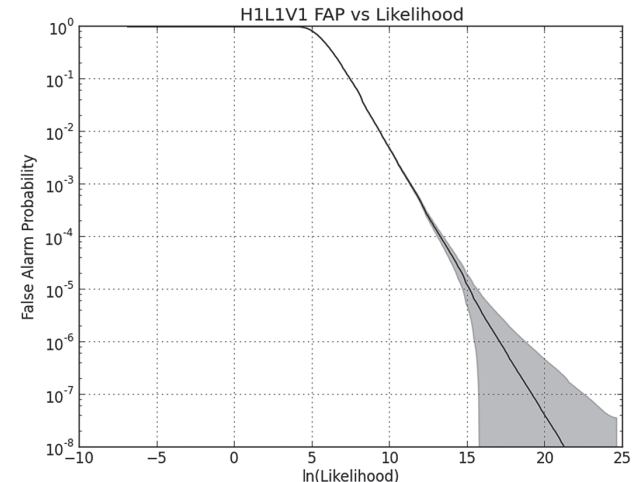

b. An example FAP vs. Log likelihood plot.

Figure 5. Likelihood distribution in noise and FAP in an example pipeline run.

\section{TESTING THE GSTLAL PIPELINE}

In order to have the optimal analysis pipeline ready when Advanced LIGO (aLIGO) turns on, we test the GSTLAL analysis pipeline by running it with a collection of stochastic banks and injections of simulated signal added to recolored S6 data. Recolored S6 data means real Science Run 6 data, but strips out the PSDs from iLIGO and recolors the data with the Early aLIGO noise curve. Shown in Figure 6 is the aLIGO Amplitude Spectral Density (ASD), the square root of the PSD. We then evaluate the pipeline efficiency through injection accuracy, missed and found plots, horizon distance, SNR vs. $\chi^{2}$, FAP, pipeline run time, etc. In this section, we are only concerned with the pipeline search sensitivity, characterized by different detection statistics, setting aside the computational run time. In addition to search sensitivity analysis, in the next section, we will analyze the pipeline run time for one day of data, trying to minimize the run time without jeopardizing the detection efficiency. 


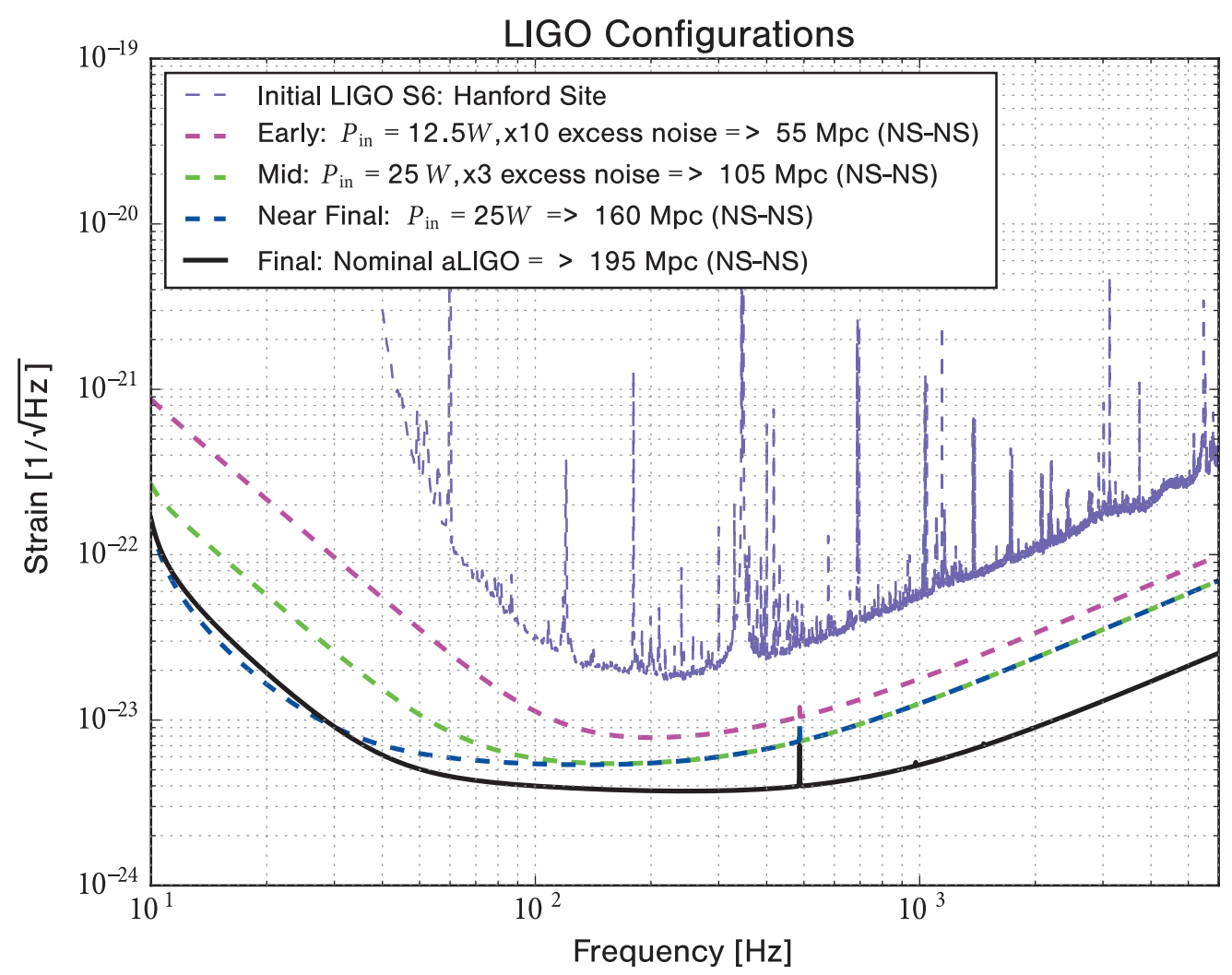

Figure 6. LIGO Amplitude Spectral Density curves. The pink dash line is the ASD model of Early aLIGO.

\section{CURRENT RUNS STATUS}

We investigate different aspects of the GSTLAL analysis pipeline with several stochastic template banks, varied by spin range, minimum integrating frequency, bank shape, etc. We have finished generating a collection of stochastic template banks, with parameter space listed in Table 1. In Table 1, the last two banks, LgSq and LgTrape result from stitching dense low mass banks together with sparse high mass banks. Adding in higher mass region does not significantly increase the total number of templates, since BBH systems with higher mass tend to have shorter waveforms as shown in the simple example in Figure 7, leading to wider coverage of mass parameter space for a specified minimal match. Simultaneously, other group members are trying to push down to lower mass region with a minimum component mass of $3 M_{\odot}$. We will then combine these efforts to generate banks that cover from $6 M_{\odot}$ to $110 M_{\odot}$ in total mass, and run the pipeline with them. In addition to mass, by comparing Bank Sq2 and Bank Sq3, we demonstrate the significant contribution of spins to the number of templates in a bank. The effects of including spins on number of templates in banks is also shown in Figure 8. 


\begin{tabular}{|c|c|c|c|c|c|c|c|c|}
\hline & Shape & $\begin{array}{c}\text { Number } \\
\text { of } \\
\text { Templates }\end{array}$ & $\begin{array}{c}\text { Compo- } \\
\text { nent Mass }\end{array}$ & $\begin{array}{c}\text { Total } \\
\text { Mass }\end{array}$ & $\begin{array}{c}\text { Mass } \\
\text { Ratio }\end{array}$ & F-low & Spin & $\begin{array}{c}\text { Rejection } \\
\text { Rate }\end{array}$ \\
\hline Sq1 & square & 13713 & {$[4.9,22]$} & {$[9.8,44]$} & {$[1,4]$} & $20 \mathrm{~Hz}$ & {$[-0.9,0.9]$} & 100 \\
\hline Sq2 & square & 27430 & {$[4.9,22]$} & {$[9.8,44]$} & {$[1,4]$} & $20 \mathrm{~Hz}$ & {$[-0.9,0.9]$} & 1000 \\
\hline Sq3 & square & 18370 & {$[4.9,22]$} & {$[9.8,44]$} & {$[1,4]$} & $20 \mathrm{~Hz}$ & {$[-0.2,0.9]$} & 1000 \\
\hline Sq4 & square & 12237 & {$[4.9,22]$} & {$[9.8,44]$} & {$[1,4]$} & $30 \mathrm{~Hz}$ & {$[-0.9,0.9]$} & 1000 \\
\hline Trape5 & trapezoid & 32289 & {$[1,44]$} & {$[9.8,44]$} & {$[1,4]$} & $30 \mathrm{~Hz}$ & {$[-0.9,0.9]$} & 1000 \\
\hline Trape6 & trapezoid & 100713 & {$[1,44]$} & {$[9.8,44]$} & {$[1,4]$} & $20 \mathrm{~Hz}$ & {$[-0.9,0.9]$} & 1000 \\
\hline LgSq & square & 13561 & {$[4.9,22]$} & {$[9.8,110]$} & {$[1,4]$} & $30 \mathrm{~Hz}$ & {$[-0.9,0.9]$} & 1000 \\
\hline LgTrape & trapezoid & 32597 & {$[1,110]$} & {$[9.8,110]$} & {$[1,4]$} & $30 \mathrm{~Hz}$ & {$[-0.9,0.9]$} & 1000 \\
\hline
\end{tabular}

Table 1. Bank parameter space

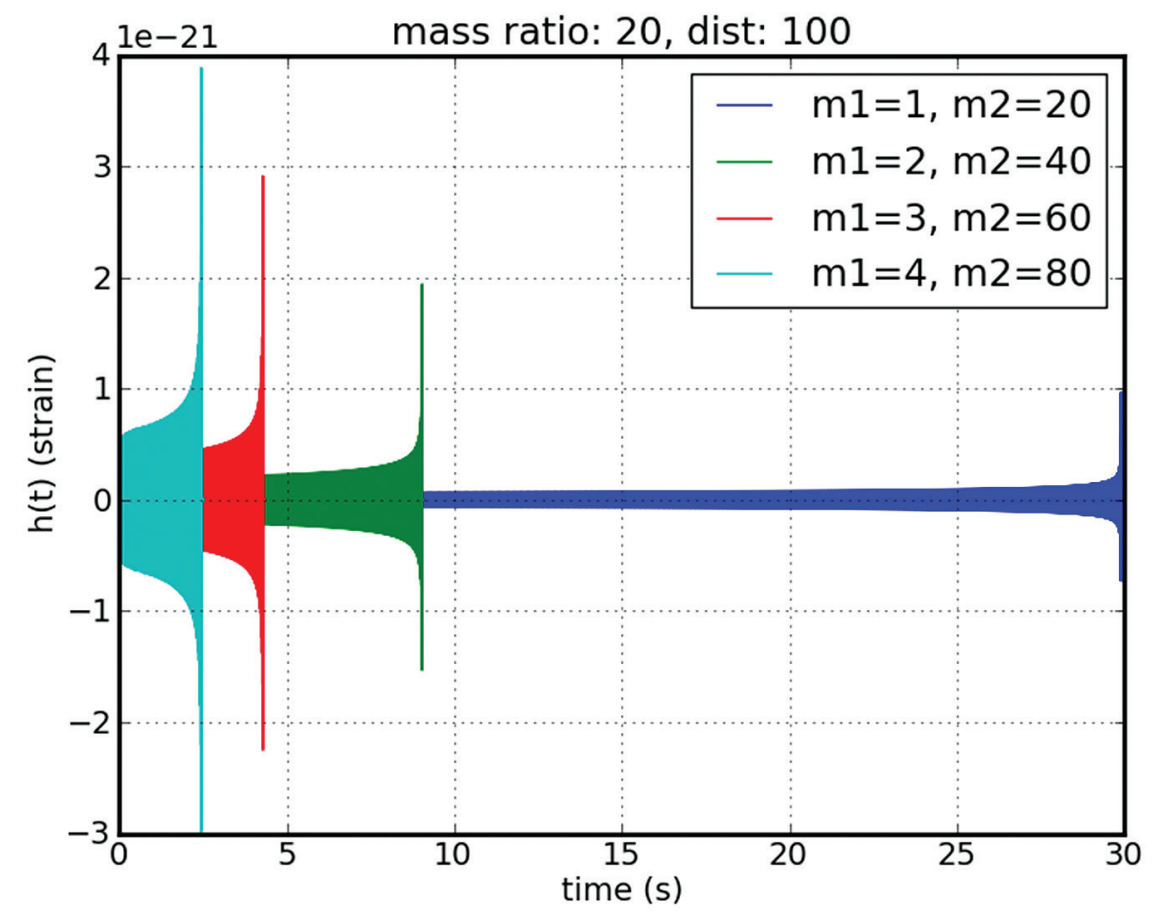

Figure 7. Example GW waveforms. Higher-mass BBH systems have shorter waveform durations above a specified $f_{\min }=20 \mathrm{~Hz}$. 


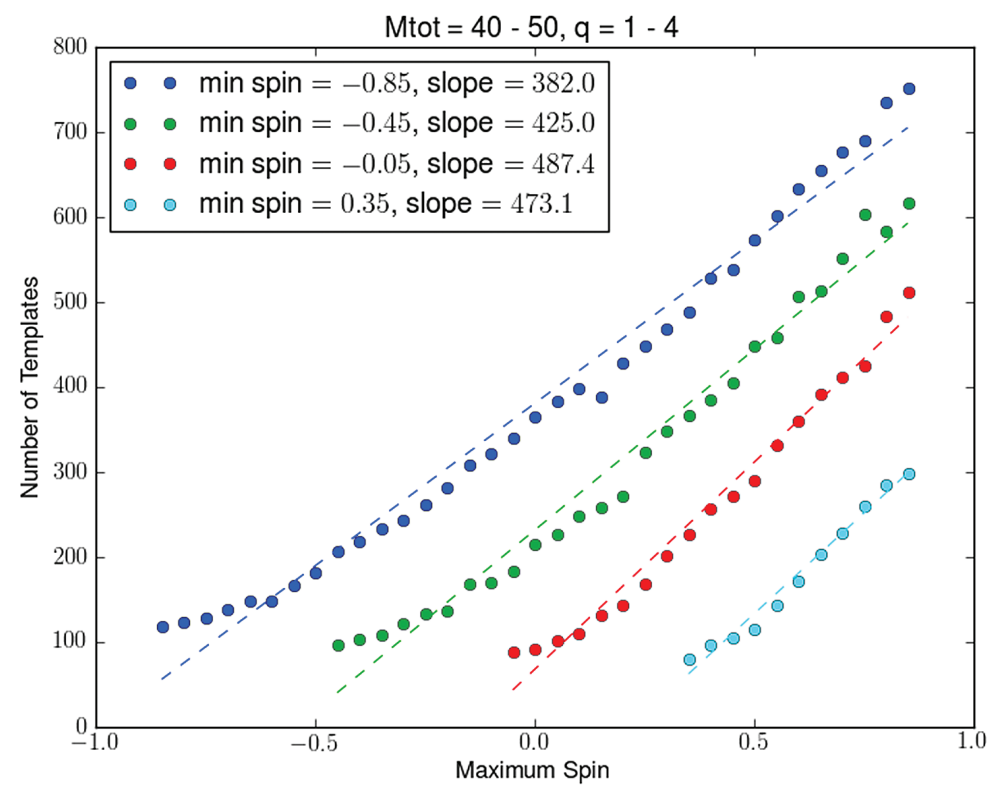

Figure 8. Range of spins vs. number of templates

We have finished a number of daily (one-day data analysis) pipeline runs with injections of recolored S6 data using these banks, and the status of the daily pipeline runs is listed in Table 2. The GPS start time of S6 is 966384015 s, and the GPS stop time of S6 is 1077667216 s. Analysis of one week of data with Bank Sq2 and Bank Trape5 is done. Our next step is to combine the daily results into weekly results. Ultimately, we hope to combine weeklies into monthlies, monthlies into yearlies, etc.

\begin{tabular}{|c|c|c|c|c|c|c|c|}
\hline & Day 1 & Day 2 & Day 3 & Day 4 & Day 5 & Day 6 & Day 7 \\
\hline $\begin{array}{c}\text { GPS Start } \\
\text { Time }\end{array}$ & 966384015 & 966470415 & 966556815 & 966643215 & 966729615 & 966816015 & 966902415 \\
\hline $\begin{array}{c}\text { GPS Stop } \\
\text { Time }\end{array}$ & 966470415 & 966556815 & 966643215 & 966729615 & 966816015 & 966902415 & 966988815 \\
\hline Sq1 & $\sqrt{ }$ & $\mathrm{X}$ & $\mathrm{X}$ & $\mathrm{X}$ & $\mathrm{X}$ & $\mathrm{X}$ & $\mathrm{X}$ \\
\hline Sq2 & $\sqrt{ }$ & $\sqrt{ }$ & $\sqrt{ }$ & $\sqrt{ }$ & $\sqrt{ }$ & $\sqrt{ }$ & $\sqrt{ }$ \\
\hline Sq3 & $\sqrt{ }$ & $\mathrm{X}$ & $\mathrm{X}$ & $\mathrm{X}$ & $\mathrm{X}$ & $\mathrm{X}$ & $\mathrm{X}$ \\
\hline Sq4 & $\sqrt{ }$ & $\mathrm{X}$ & $\mathrm{X}$ & $\mathrm{X}$ & $\mathrm{X}$ & $\mathrm{X}$ & $\mathrm{X}$ \\
\hline Trape5 & $\sqrt{ }$ & $\sqrt{ }$ & $\sqrt{ }$ & $\sqrt{ }$ & $\sqrt{ }$ & $\sqrt{ }$ & $\sqrt{ }$ \\
\hline Trape6 & $\mathrm{X}$ & $\mathrm{X}$ & $\mathrm{X}$ & $\mathrm{X}$ & $\mathrm{X}$ & $\mathrm{X}$ & $\mathrm{X}$ \\
\hline LgSq & $\sqrt{ }$ & $\Delta$ & $\Delta$ & $\Delta$ & $\Delta$ & $\Delta$ & $\Delta$ \\
\hline LgTrape & $\sqrt{ }$ & $\Delta$ & $\Delta$ & $\Delta$ & $\Delta$ & $\Delta$ & $\Delta$ \\
\hline
\end{tabular}

Table 2. GSTLAL daily runs status ( $\sqrt{ }$ : Finished; $\Delta$ : Planning; X: No plan to run.) 


\section{PUSHING UP INTO HIGH MASS REGION}

We have successfully completed pipeline runs with banks LgSq and LgTrape. These two large banks are shown in Figure 9. We could see that in the upper right high mass region, both banks are very sparse, as explained before. In Figure 10, we show histograms of bank parameters vs. number of templates. Figure 10a and Figure 10b show that we successfully cover the full spin range, and that spins distribute uniformly throughout the spin range. And both banks have much more templates in the lower mass region than in the higher mass region as shown in Figure 10e and Figure 10f. Figure $10 \mathrm{c}$ and Figure 10d illustrate that templates in square banks concentrate near the $q=1$ line, while templates in trapezoid banks gather around the $q=4$ line. Our next step is to push down into low mass region, combine into an even larger mass range, and run the pipeline.

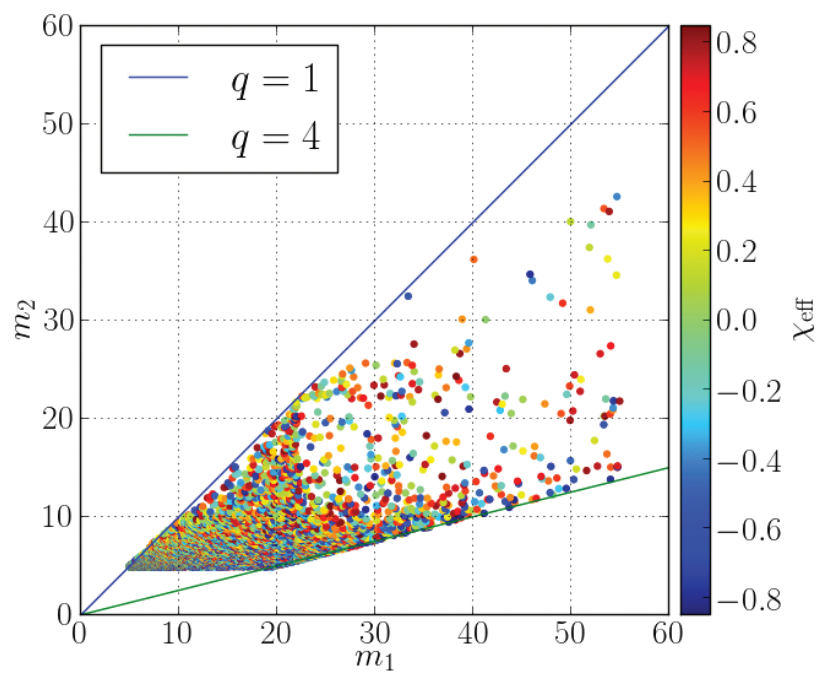

a. Large square bank

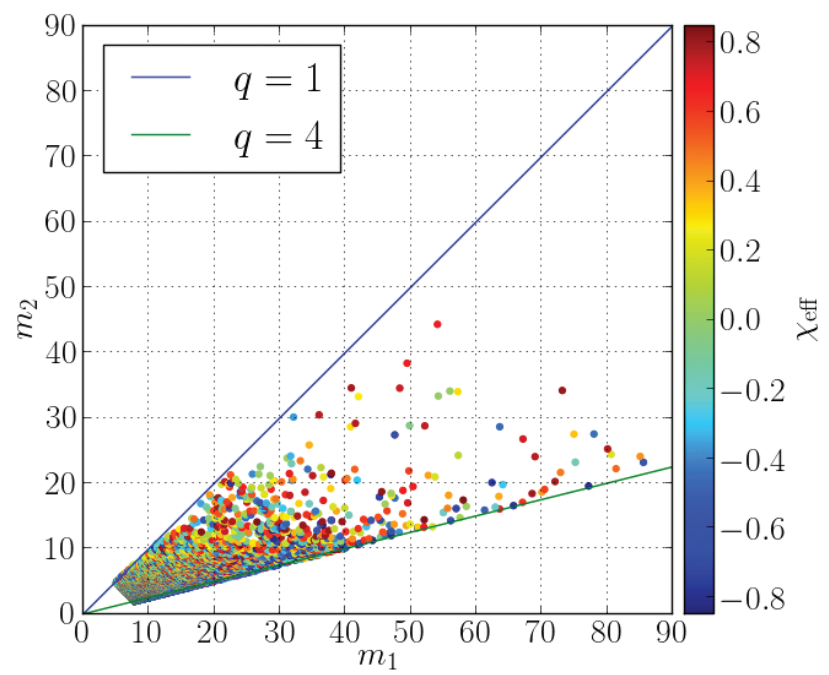

b. Large trapezoid bank

Figure 9. Visualization of large mass span banks. 


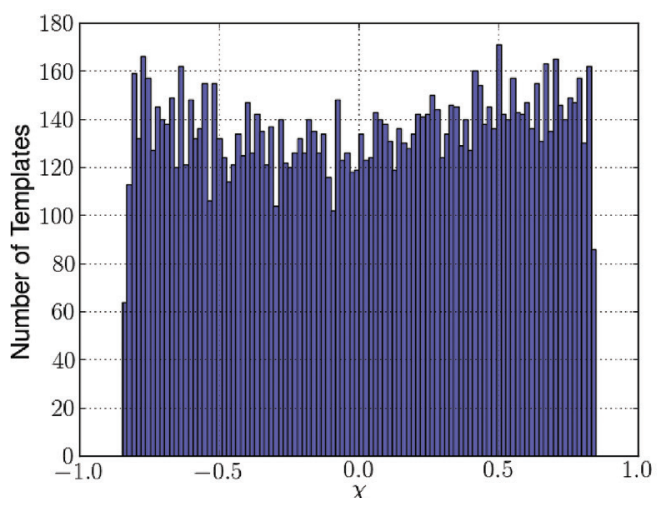

a. Square bank: $\chi$ vs. number of template

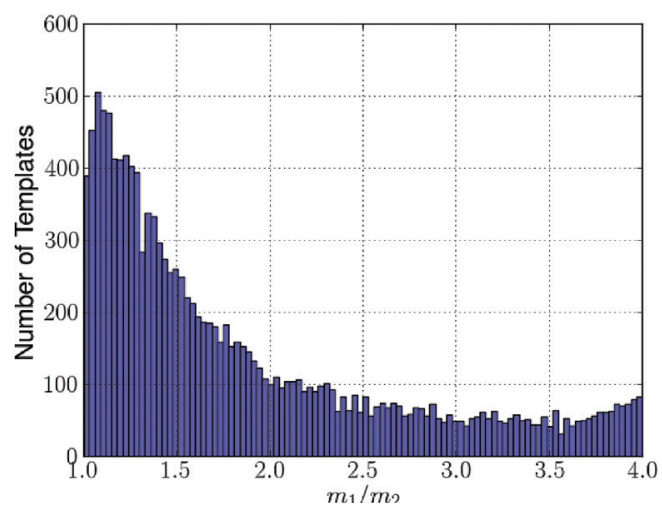

c. Square bank: mass ratio vs. number of templates

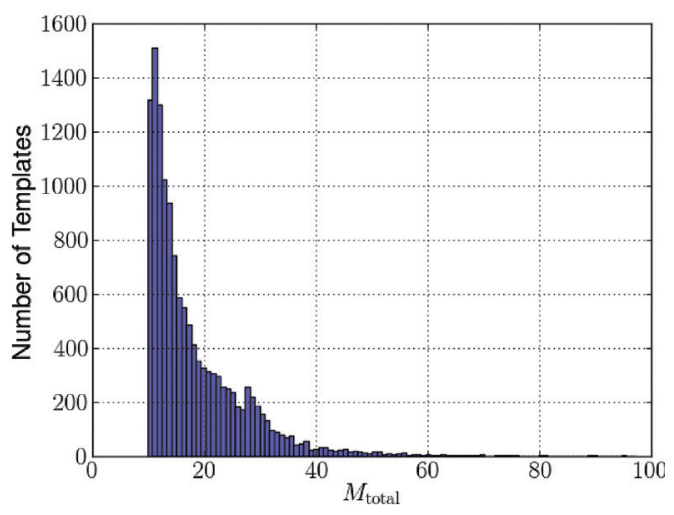

e. Square bank: total mass vs. number of templates

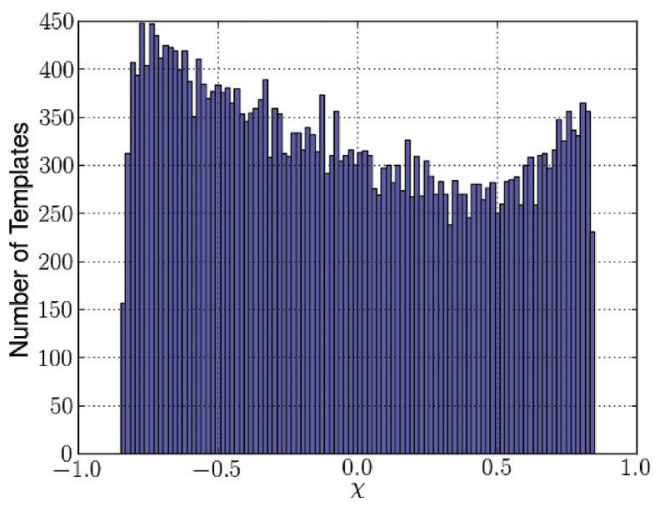

b. Trapezoid bank: $\chi$ vs. number of templates

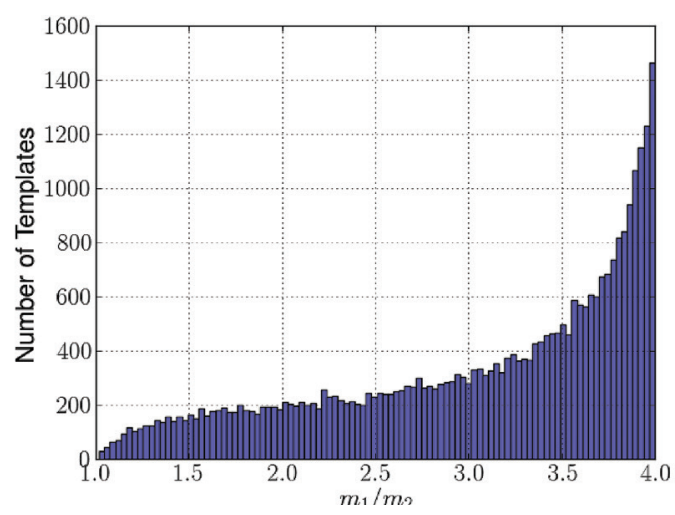

d. Trapezoid bank: mass ratio vs. number of templates

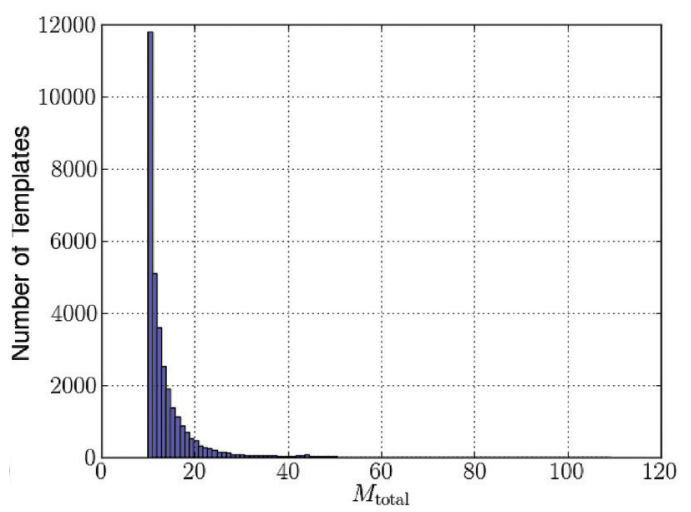

f. Trapezoid bank: total mass vs. number of templates

Figure 10. 1-D histograms of bank parameters. 


\section{EVALUATE THE PIPELINE EFFICIENCY}

This subsection briefly shows how we check the pipeline's performance. We demonstrate ways to evaluate the pipeline eciency through injection accuracy, missed and found plots, horizon distance, SNR vs. $\chi^{2}$, and FAP using Day 1 run on Bank Trape 5 as an example. The parameters of Bank Trape5 are noted in Table 1. The shape of the bank as well as certain parameters is shown in Figure 11.

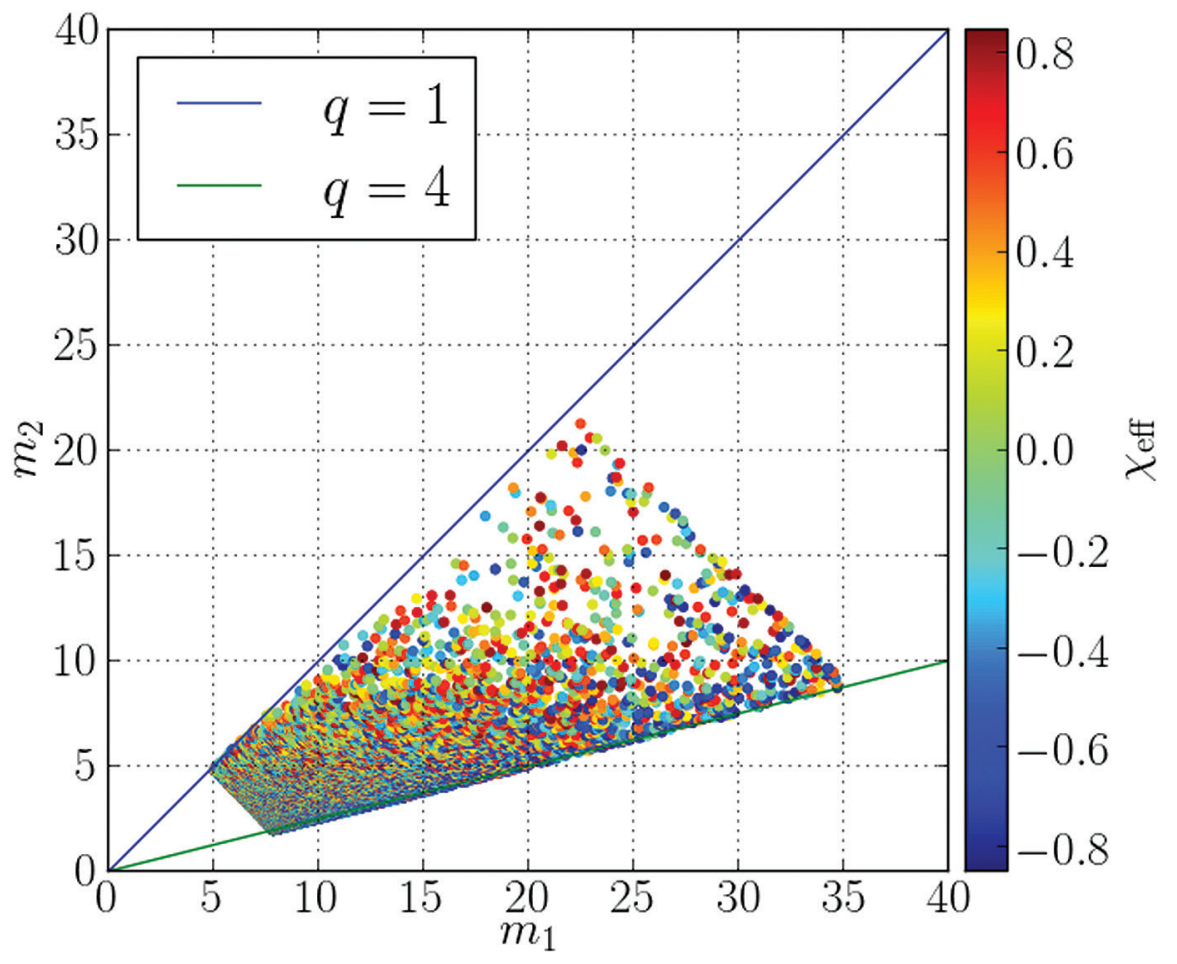

Figure 11. Visualization of Bank Trape5

\section{INJECTION ACCURACY}

We examine the pipeline's ability to recover BBH systems' parameters by checking the injection accuracy. The accuracy of recovered parameters aids in diagnosing the performance of the pipeline as well as hinting at the possibility of measuring certain parameters. As illustrated in Figure 12,we see the full spin range, the chirp mass $M_{\text {chirp }}=\left(m_{1} m_{2}\right)^{3 / 5} / M_{\mathrm{tot}}^{1 / 5}$, and the end time can be mostly recovered. Mass parameters for the low mass search are often recovered better than mass parameters for the high mass search because high mass waveforms are shorter as explained before. Degeneracy with chirp mass causes some outliers in recovering spins. Generally, most trigger parameters are well recovered, but we do have outliers that return very badly recovered parameters. At least some of these are due to "glitches" in the data masking the (weaker) signal injections. Our next step is to examine the output trigger database ("sqlite") files to dig into these events, identify the problems and try to resolve them. 


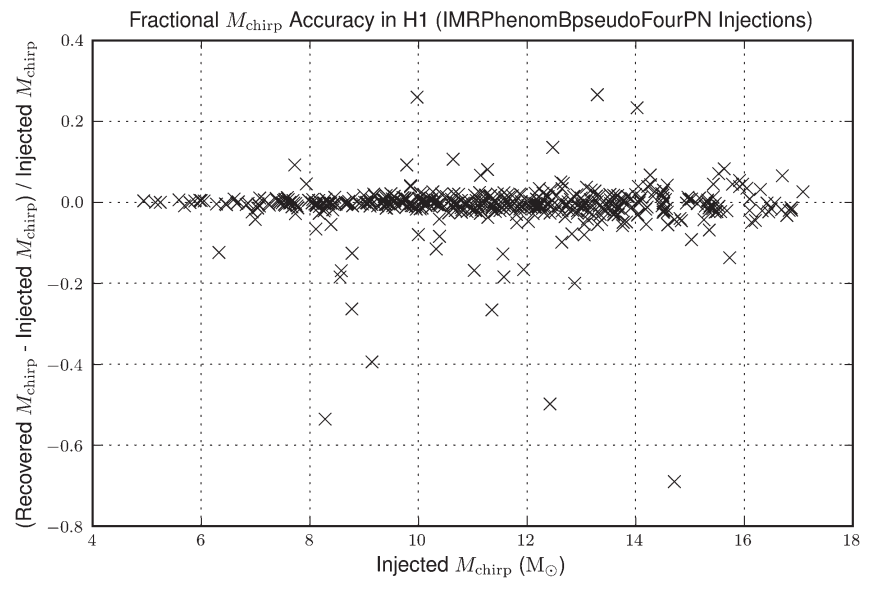

a. Injected chirp mass vs. recovered chirp mass

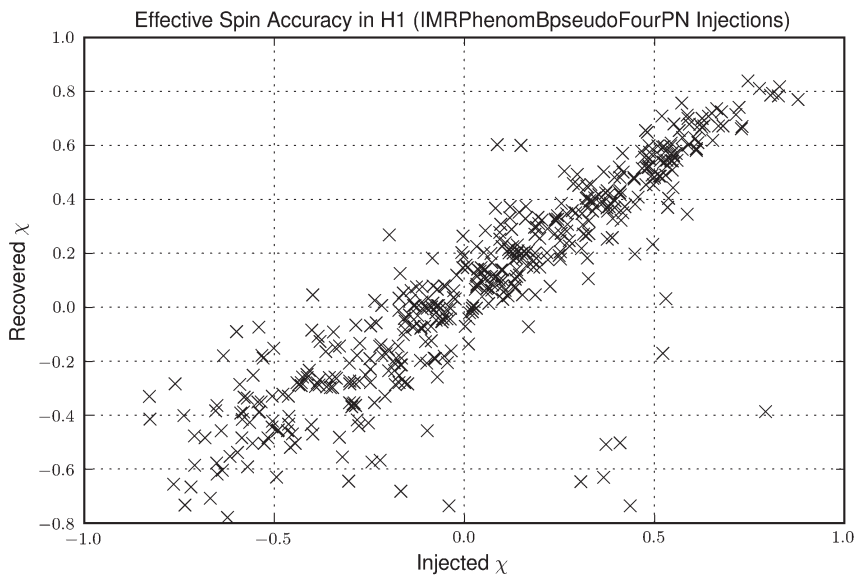

b. Injected effective spin vs. recovered effective spin

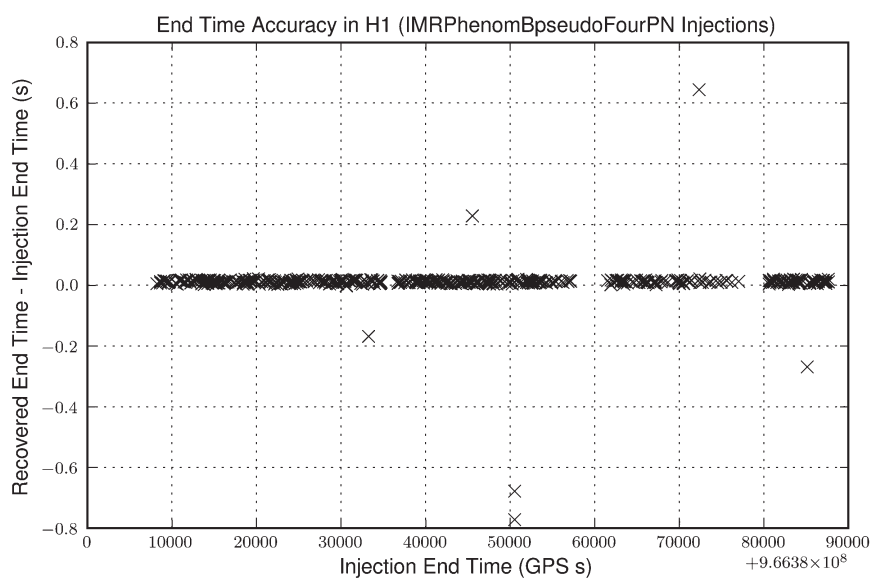

c. Injected effective time vs. recovered effective time

Figure 12. Injection accuracy. 


\section{MISSED AND FOUND}

A table listing numbers of missed and found signal injections is shown in Figure 13a. Measuring the missed and found injections as a function of various parameters aids in diagnosing the pipeline as well as providing the expected sensitivity of the pipeline to real signals. We measure the missed and found injections as a function of chirp mass, total mass, time, and spin. We here show the plot of effective distance of found and missed injections as a function of chirp mass in Figure 13b. We can see in Fig 13b that more distant signal injections are usually missed, but the dividing line between missed and found injections increases in distance with chirp mass, because the higher chirp mass sources produce higher amplitude GW signals (see also Figure 16).

\begin{tabular}{|c|c|c|}
\hline On Instruments & Participating Instruments & Missed / Found \\
\hline H1V1 & H1V1 & FOUND: 33 \\
\hline H1V1 & - & MISSED: 376 \\
\hline H1L1V1 & H1L1 & FOUND: 379 \\
\hline H1L1V1 & H1L1V1 & FOUND: 149 \\
\hline H1L1V1 & trapezoid & FOUND: 9 \\
\hline H1L1V1 & H1L1 & FOUND: 4 \\
\hline H1L1V1 & L1V1 & MISSED: 1125 \\
\hline H1L1 & H1L1 & FOUND: 14 \\
\hline H1L1 & - & MISSED: 28 \\
\hline L1V1 & L1V1 & FOUND: 20 \\
\hline L1V1 & - & MISSED: 212 \\
\hline
\end{tabular}

a. Summary of missed and found injections broken up by detector time

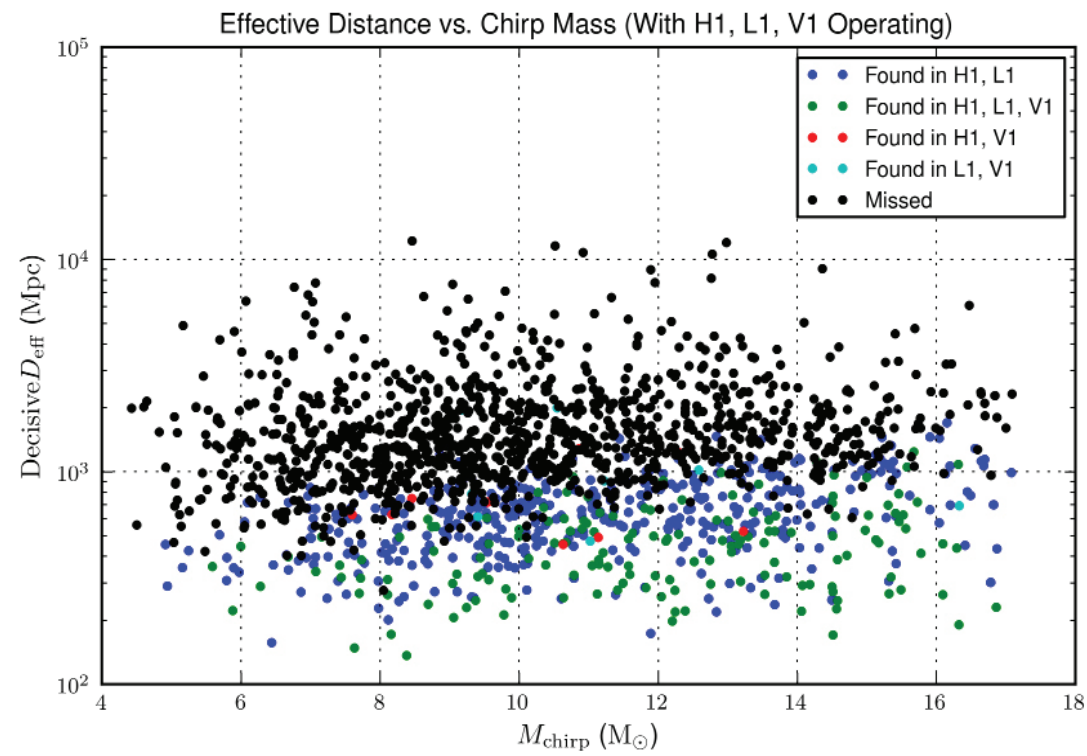

b. Effective distance of found and missed injections as a function of chirp mass

Figure 13. Missed and found table and plot 


\section{HORIZON DISTANCE}

The horizon distance, which is the furthest distance at which you could see a compact binary system at a given SNR, is another useful pipeline sanity check. Horizon distance grows with total mass, since the gravitational waves are louder for higher mass systems; but above a certain total mass, it will "turn over", ie, start falling (to zero) at higher masses, as the merger frequency falls below the LIGO frequency band $\left(f_{\min }\right)$. Example horizon distance plots generated from pipeline runs are shown in Figure 14, which gives the distance at which you should see a $1.4 M_{\odot}+1.4 M_{\odot}$ binary neutron star (BNS) system at SNR 8.
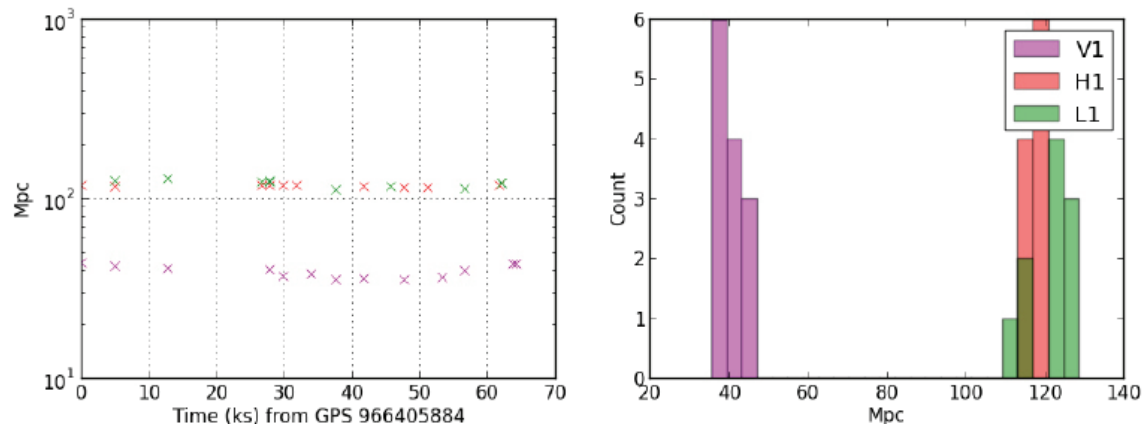

Figure 14. Horizon distance for a $1.4 M_{\odot}+1.4 M_{\odot}$ BNS system at $\mathrm{SNR}=8$

The first panel shows the time evolution of the horizon distance and the second panel shows the same information in histogram for the two LIGO detectors participating in S6 and the VIRGO detector participating in VSR3.

Since horizon distance information for high-mass BBH systems is missing, efforts were put into producing plots that are more informative for higher total mass BBH systems. We use an equivalent quantity, mean sensitive distance, which is the horizon distance averaged over all sky position and orientation angles, to characterize the overall sensitivity of the search. From this averaging, the mean sensitive distance is equal to the horizon distance divided by 2.26 . To evaluate the sensitivity, we first compute the theoretical mean sensitive distance using the simulated real detector Amplitude Spectral Density (ASD) curve generated from Early aLIGO ASD model. Then we compare this theoretical mean sensitive distance with the search range computed in the pipeline. The Early aLIGO ASD model and an example simulated real detector Early aLIGO ASD curve are shown in Figure 6 and Figure 15. Using the simulated detector ASD better models the theoretical mean sensitive distance than using the Early aLIGO ASD model because it takes into account the detector non-stationarity, non-Gaussianity, and instrumental spectral lines. 


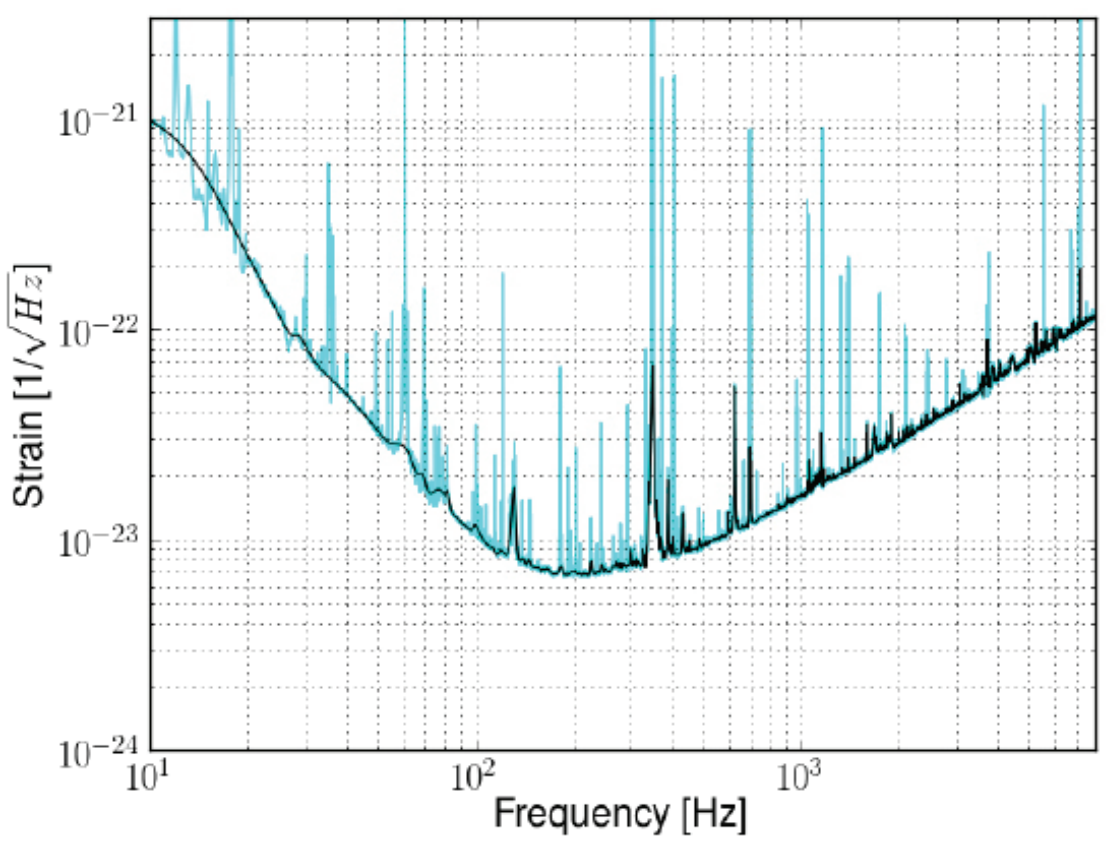

Figure 15. An example smoothed simulated real detector ASD curve generated from Early aLIGO ASDmodel in a pipeline run.

Finally, with the theoretical mean sensitive distance computed (see Figure 16a), we estimate the search sensitivity by comparing the theoretical plot with the search sensitivity plot from the pipeline run (see Figure 16 for the Day 1 run on Bank Trape5), as a visual sanity check. If our pipeline is sensitive enough, then the pipeline mean sensitive distance should match with the theoretical one computed from real detector ASDs.

As an example, for a $\mathrm{BBH}$ system with a total mass of $\sim 21 M_{\odot}$, the theoretical mean sensitive distance is roughly $230 \sim 260 \mathrm{Mpc}$ indicated in Figure 16a. In Figure 16, $\sim 21 M_{\odot}$ roughly points at the middle of the green segment, and this point suggests a pipeline mean sensitive distance of $240 \sim 260 \mathrm{Mpc}$. These comparisons provide us a first check of the pipeline. And in this case, the sensitivity with the real search pipeline is quite comparable to expectations; the pipeline works as expected. And further examinations of the pipeline will be carried out with detailed statistics and more informative plots. 


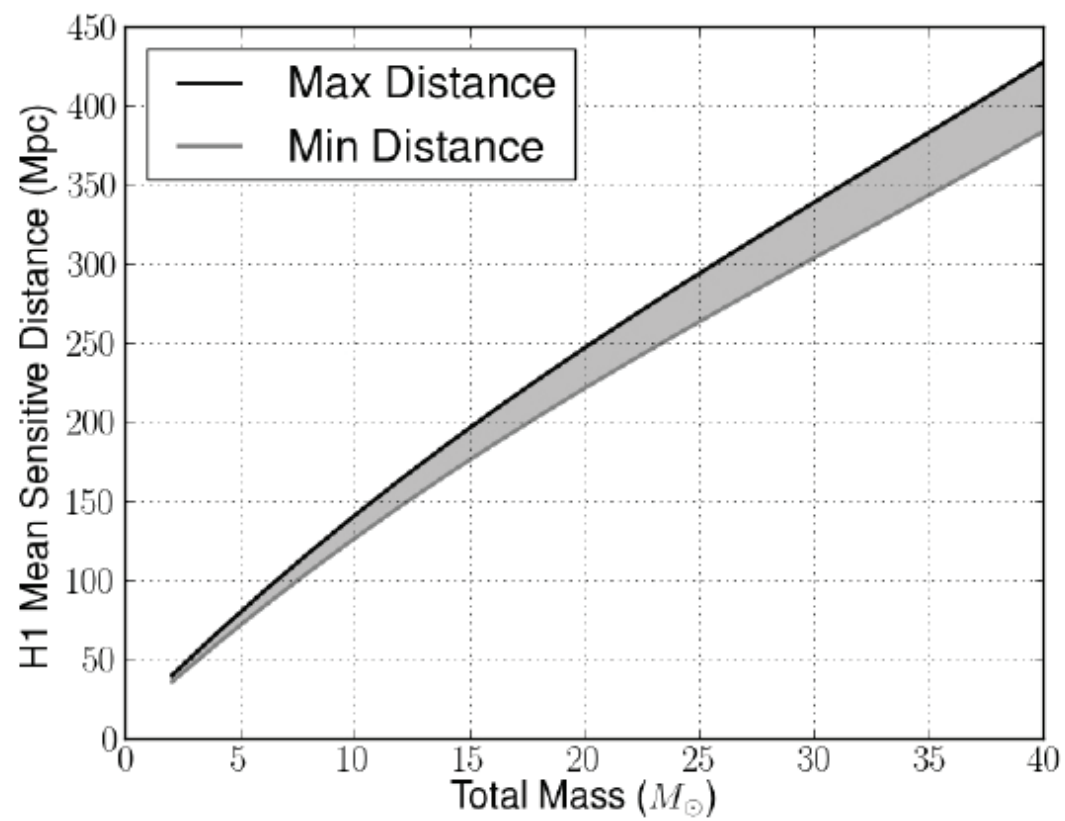

a. Theoretical mean sensitive distance

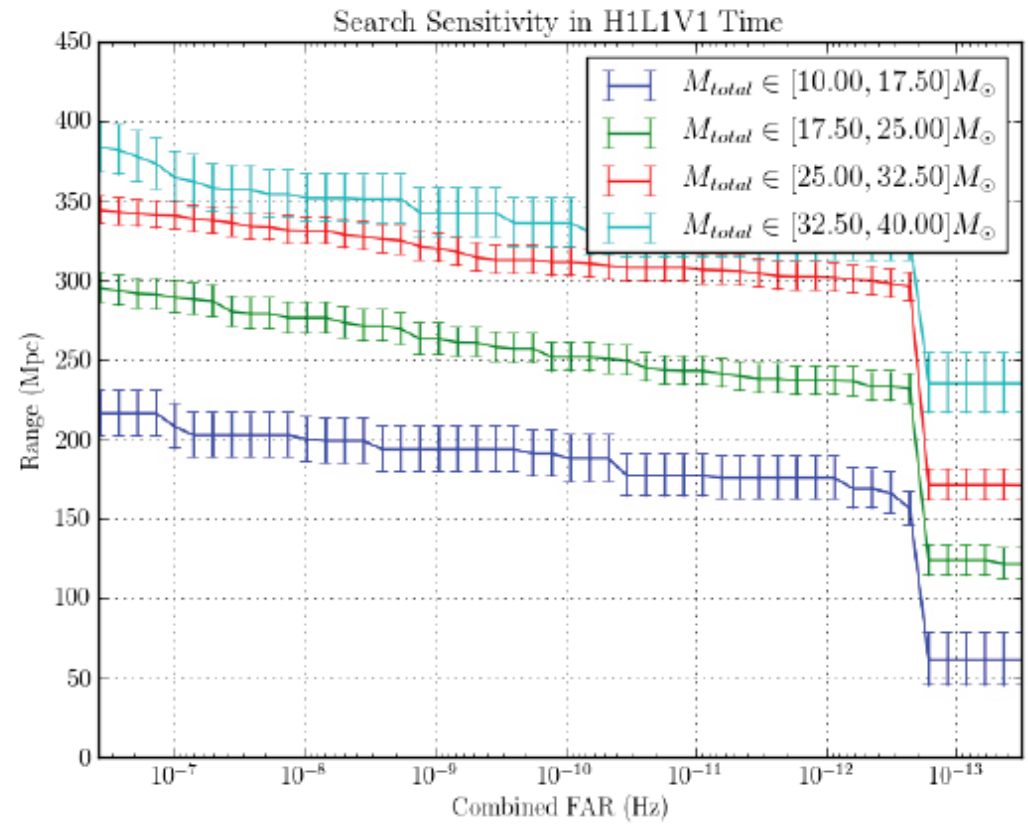

b. Pipeline search range

Figure 16. Theoretical and real pipeline mean sensitive distance 


\section{TIMING ANALYSIS}

Timing is another crucial aspect in the GSTLAL analysis pipeline. Undoubtedly, we would like to cover as large a parameter space as possible. However, this idealized case will inescapably introduce a large number of templates into a bank and consequently the pipeline will take a long time to finish. Therefore, it is not possible to enlarge the parameter space indefinitely. With limited resources, we would like to approach a sufficiently large parameter space to increase detection possibility within reasonable search time. Therefore, investigation of template placements in banks and pipeline timing analysis is paramount. In this section, we will discuss our analysis of the execution time of our pipeline. This analysis is still ongoing at an early stage. We will provide the progresses that we had so far.

The entire workflow runs under the Condor job control system, and is constructed as a Condor DAG. ${ }^{13}$ Through carefully generating and analyzing program execution time logs, we can access the pipeline run time information after the runs have finished. More importantly, we can scrutinize the jobs that slow down the whole pipeline and try to tune the pipeline parameters so as to speed up the whole process. For timing analysis, we have three different times recorded, "wall time", "good time", and "CPU usage". "Wall time" is the difference between the time at which a task finishes and the time at which the task started, including time that passes due to artificial delays or waiting for resources to become available. "Good time" is the allocated time which contributes to the completion of the job. "CPU usage" is the CPU time which contributes to the completion of this job. Here we show the "wall time" analysis results from one example pipeline run, which is the Day 1 data of S6 using Bank Trape5 referred in Table 1. The analysis of the other two time types is similar, and we include the information in the pipeline runs summary page.

Figure 17 and Figure 18 both show the program flowchart of the GSTLAL pipeline. A child process could not start if the information provided by its parent process is not ready. Figure 17 shows the maximum "wall time" of each process, and Figure 18 shows the mean "wall time" \pm standard deviation of each process. Comparing the two flow charts, we see some large discrepancies between the process's max time and mean time. For example, "gstlal_inspiral" has a mean time of 33 minutes, while having a max time of 3 hours. To better visualize the run time distribution, we generate histograms of different processes, which are shown in Figure 19. We notice in Figure 19 that some processes such as "gstlal_inspiral" in Figure 19a have long tails of run times. "gstlal_inspiral" is one of the core processes that have many jobs (in Figure 17, there are 1960 gstlal_inspiral jobs) and this spread significantly slows down the whole pipeline run. Ultimately, we would want all the jobs from the same program to finish close in time. In order to achieve this optimization, we will probe the job information by examining the trigger database sqlite files. On one hand, we can reorder the job execution waiting line so that jobs taking the longest time start first; on the other hand, we can split the jobs in a smarter way which will equalize the run time for each job. Subsequently, jobs in line for the next process can start around the same time, greatly minimizing the total run time. 


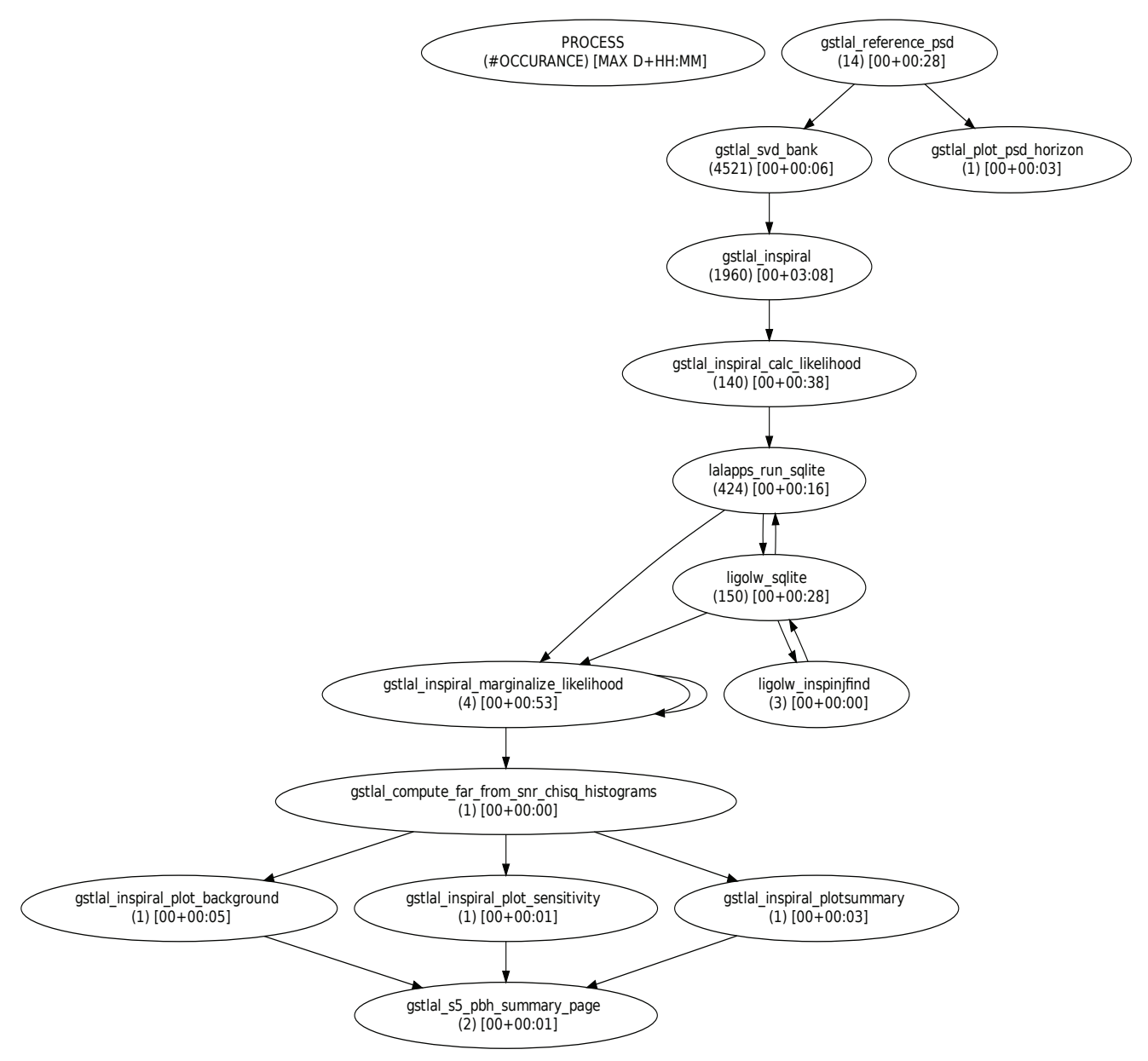

Figure 17. Example flow chart of max process run times. 


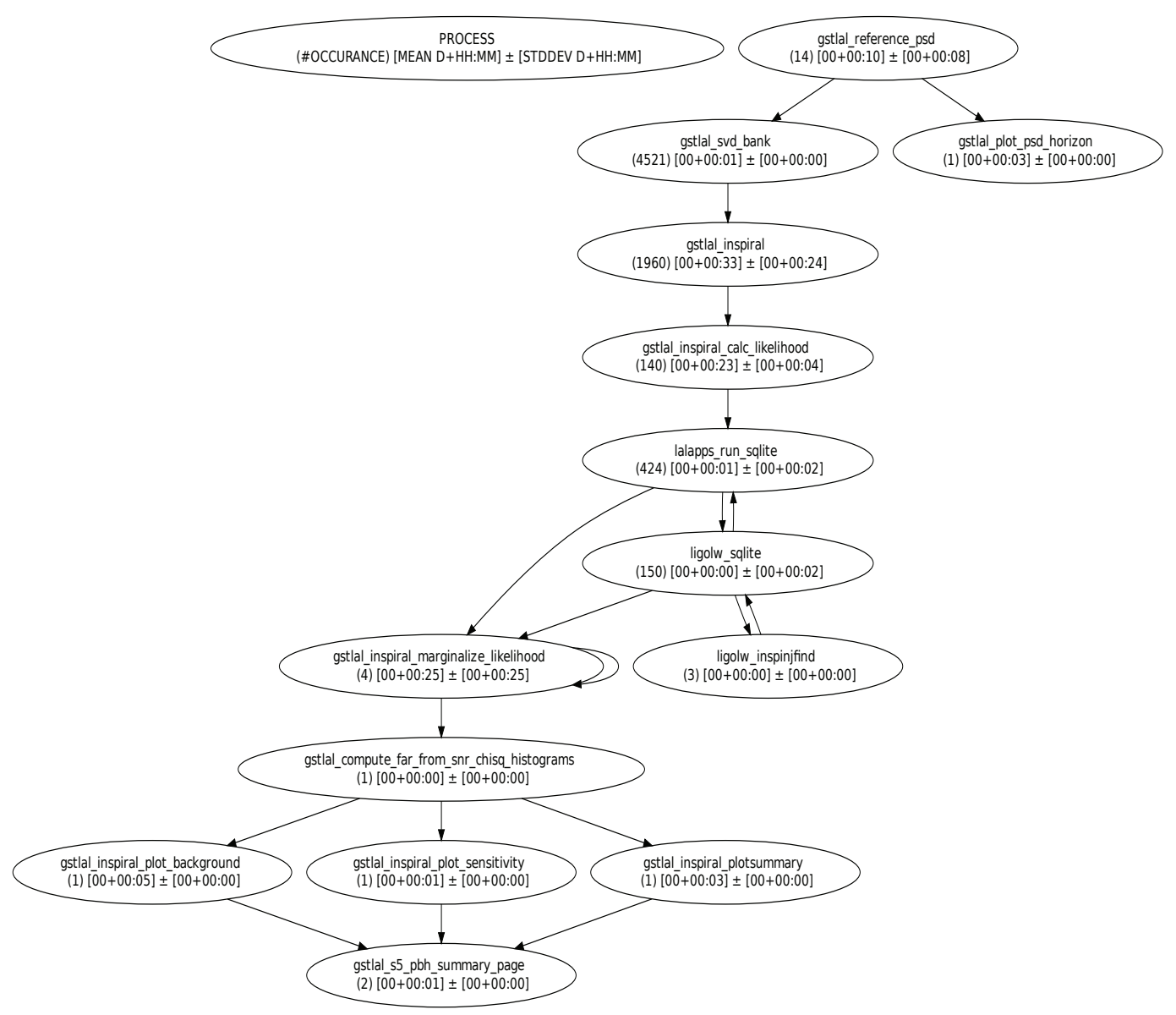

Figure 18. Example flow chart of mean process run times. 


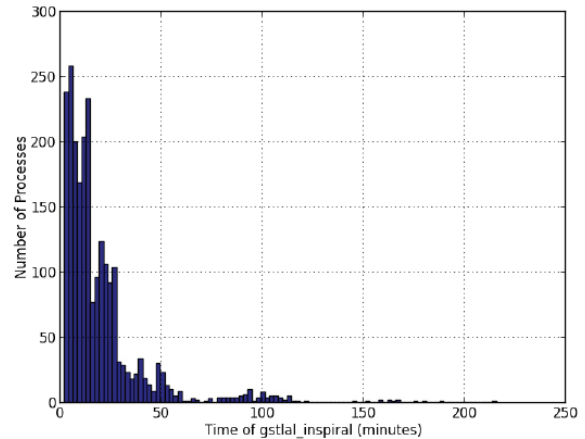

a. gstlal_inspiral

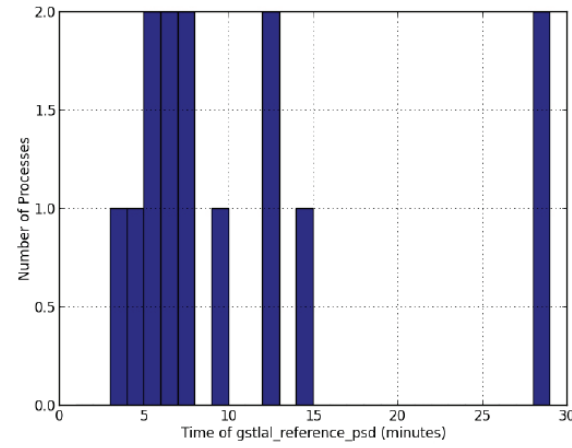

c. gstlal_reference_psd

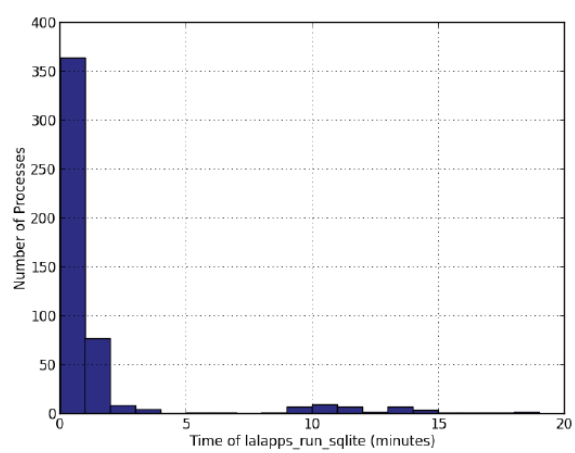

e. lalapps_run_sqlite

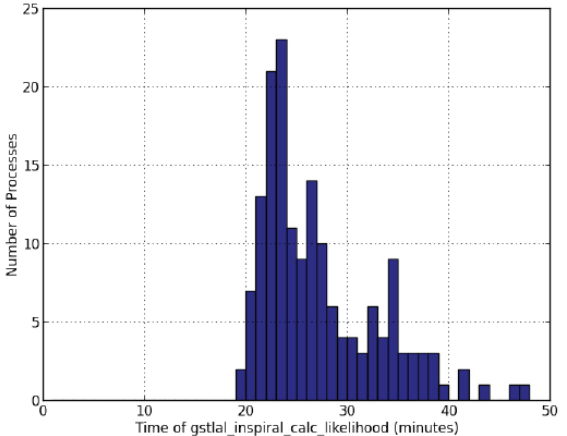

b. gstlal_inspiral_calc_likelihood

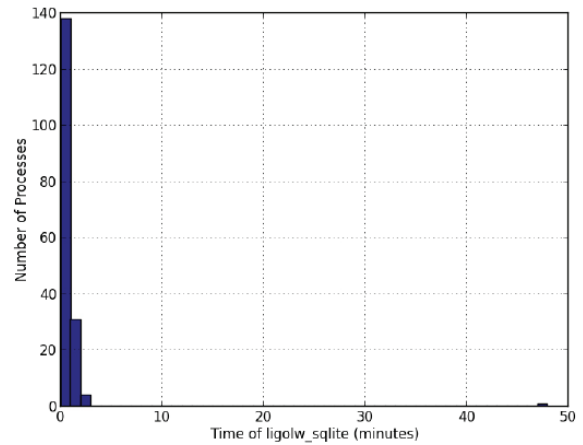

d. ligolw_sqlite

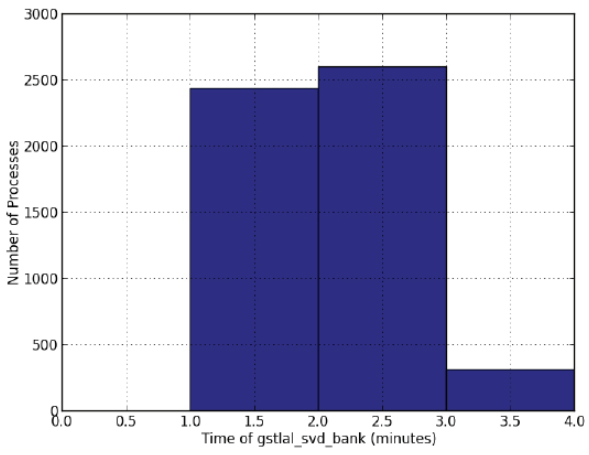

f. gstlal_svd_bank

Figure 19. 1-D histograms of run times of processes which occur more than 10 times in the pipeline runs. 


\section{FUTURE WORK}

Now that we have a collection of stochastic banks and daily runs, our next steps are to fully debug and tune the GSTLAL pipeline to improve its sensitivity and assemble a complete suite of diagnostic plots and search summary information.

Daily analysis of the first week of data in S6 with Bank Sq2 and Bank Trape5 is done. Up next, we will combine dailies into weeklies, weeklies into monthlies, monthlies into yearlies, etc. To make weeklies, we combine daily coincident triggers together and recalculate the background level. PSDs of GW detectors remain relatively stable over the span of one week. Thus, weeklies enable us to better estimate backgrounds since more data are available to better separate signal from noise. Each weekly summary is a "detection trial"; over many weeks, there are many trials, and thus many opportunities for noise fluctuations to fake signals. Combining them into monthlies and yearlies, and eventually, one summary over the whole run gives us one trial for finding a signal above the noise (no "trials factor").

Pipeline runs using injections containing only Gaussian noise are ongoing now. We want to compare the pipeline efficiency of the existing runs with that of Gaussian runs to see how "glitches" affect the pipeline's efficiency. For further analysis regarding existing runs, we will investigate the outliers that give us bad injection accuracy or exceptionally long job run time. Ultimately, we want to tune the pipeline parameters to improve the its performance, in terms of efficiency and pipeline run time so that we can arrive at optimal settings under different anticipated noise fluctuation conditions for aLIGO.

\section{ACKNOWLEDGMENTS}

I would like to express my gratitude to my research mentor, Professor Alan J. Weinstein for giving me the great opportunity of studying and working in the LIGO CBC-BBH working group during the summer in 2014 as a participant of the Caltech LIGO SURF program. I would also like to thank Caltech LIGO postdoc Tjonnie G. F. Li and graduate student Surabhi Sachdev for all the help and advice during the summer. I would not have made so much progress without their help. In addition, I am grateful to all the staff at Caltech, the personnel in LIGO Scientific Collaboration, and my colleagues in LIGO CBC-BBH group, Dr. Kent Blackburn, Dr. Stephen Privitera, and Sara Frederick. Finally, I would like to express my greatest appreciation to Caltech SFP Oce and NSF for funding. 


\section{REFERENCES}

[1] B.S. Sathyaprakash, Bernard F. Schutz, Physics, Astrophysics and Cosmology with Gravitational Waves, Living Rev. Relativity 12 (2009), [DOI:10.12942/Irr-2009-2].

[2] Joel M.Weisberg, Joseph H. Taylor, Relativistic Binary Pulsar B1913+16: Thirty Years of Observations and Analysis, ASP Conf. Ser. 328 (2005) 25, [astro-ph/0407149].

[3] LIGO Scientic and Virgo Collaborations, J. Aasi, J. Abadie, B. P. Abbott, R. Abbott, T. D. Abbott, M. Abernathy, T. Accadia, F. Acernese, C. Adams, T. Adams, P. Addesso, R. Adhikari, C. Affeldt, M. Agathos, K. Agatsuma, P. Ajith, B. Allen, A. Allocca, E. Amador Ceron, D. Amariutei, S. B. Anderson, W. G. Anderson, K. Arai, M. C. Araya, S. Ast, S. M. Aston, P. Astone, D. Atkinson, P. Aufmuth, C. Aulbert, B. E. Aylott, S. Babak, P. Baker, G. Ballardin, S. Ballmer, Y. Bao, J. C. B. Barayoga, D. Barker, F. Barone, B. Barr, L. Barsotti, M. Barsuglia, M. A. Barton, I. Bartos, R. Bassiri, M. Bastarrika, A. Basti, J. Batch, J. Bauchrowitz, Th. S. Bauer, M. Bebronne, D. Beck, B. Behnke, M. Bejger, M.G. Beker, A. S. Bell, C. Bell, I. Belopolski, M. Benacquista, J. M. Berliner, A. Bertolini, et al. (740 additional authors not shown)., Search for Gravitational Waves from Binary Black Hole Inspiral, Merger and Ringdown in LIGO-Virgo Data from 2009-2010, Phys. Rev. D87 (2013) 022002, [arXiv:1209.6533].

[4] Advanced LIGO Team, Advanced LIGO reference design, LIGO technical document LIGO-M06005610-M, available at http://www.ligo.caltech.edu/docs/M/M060056-10.pdf (accessed May 2015)

[5] LIGO Scientic and Virgo Collaborations, J. Aasi, J. Abadie, B. P. Abbott, R. Abbott, T. D. Abbott, M. Abernathy, T. Accadia, F. Acernese, C. Adams, T. Adams, P. Addesso, R. Adhikari, C. Affeldt, M. Agathos, K. Agatsuma, P. Ajith, B. Allen, A. Allocca, E. Amador Ceron, D. Amariutei, S. B. Anderson, W. G. Anderson, K. Arai, M. C. Araya, S. Ast, S. M. Aston, P. Astone, D. Atkinson, P. Aufmuth, C. Aulbert, B. E. Aylott, S. Babak, P. Baker, G. Ballardin, S. Ballmer, Y. Bao, J. C. B. Barayoga, D. Barker, F. Barone, B. Barr, L. Barsotti, M. Barsuglia, M. A. Barton, I. Bartos, R. Bassiri, M. Bastarrika, A. Basti, J. Batch, J. Bauchrowitz, Th. S. Bauer, M. Bebronne, D. Beck, B. Behnke, M. Bejger, M.G. Beker, A. S. Bell, C. Bell, I. Belopolski, M. Benacquista, J. M. Berliner, A. Bertolini, et al. (740 additional authors not shown)., Search for gravitational waves from binary black hole inspiral, merger and ringdown, Phys. Rev. D83 (2011) 122005, Erratum-ibid. D86 (2012) 069903, [arXiv:1102.3781].

[6] S. Babak, R. Biswas, P. R. Brady, D. A. Brown, K. Cannon, C. D. Capano, J. H. Clayton, T. Cokelaer, J. D. E. Creighton, T. Dent, A. Dietz, S. Fairhurst, N. Fotopoulos, G. Gonzlez, C. Hanna, I. W. Harry, G. Jones, D. Keppel, D. J. A. McKechan, L. Pekowsky, S. Privitera, C. Robinson, A. C. Rodriguez, B. S. Sathyaprakash, A. S. Sengupta, M. Vallisneri, R. Vaulin, and A. J. Weinstein, Searching for Gravitational Waves from Binary Coalescence, Phys. Rev. D87 (2013) 024033, [arXiv:1208.3491].

[7] C. W. Helstrom, Statistical Theory of Signal Detection, 2nd ed. (Permagon, London, 1968).

[8] B. Allen, W. Anderson, P. Brady, D. Brown, J. Creighton, FINDCHIRP: An Algorithm for detection of gravitational waves from inspiraling compact binaries, Phys. Rev. D85 (2012) 122006, [DOI:10.1103/ PhysRevD.85.122006].

[9] S. Privitera, S. R. P. Mohapatra, P. Ajith, K. Cannon, N. Fotopoulos, M. A. Frei, C. Hanna, A. J. Weinstein, J. T. Whelan, Improving the Sensitivity of a Search for Coalescing Binary Black Holes with Non-precessing Spins in Gravitational Wave Data, Phys. Rev. D89 (2014) 024003, [arXiv:1310.5633].

[10] K. Cannon, C. Hanna, D. Keppel. Method to Estimate the Significance of Coincident Gravitational-wave Observations from Compact Binary Coalescence, Phys. Rev. D88 (2013) 024025, [DOI: 10.1103/ Phys-RevD.88.024025].

[11] B. Allen, A $\chi^{2}$ time-frequency discriminator for gravitational wave detection, Phys. Rev. D71 (2005) 062001, [arXiv:gr-qc/0405045].

[12] Early aLIGO Configurations: example scenarios toward design sensitivity, https://dcc.ligo.org/ LIGO-T1200307/public (accessed May 2015)

[13] DAGMan https://research.cs.wisc.edu/htcondor/dagman/dagman.html (accessed May 2015) 


\section{ABOUT THE STUDENT AUTHOR}

Liting Xiao is a fourth year Astronomy-Physics and Mathematics student at the University of Virginia. After graduation, she plans to pursue a Ph.D. degree in physics, specialized in experimental particle physics, astrophysics and cosmology. In the past, she has worked on Indirect Dark Matter Searches in the NOVA Experiment at Fermi National Accelerator Laboratory, student-led JefferSat Cosmic Ray Mission funded by NASA, and LIGO Gravitational Waves Searches at the California Institute of Technology. She is currently investigating time evolution of the population of compact galaxy groups in different epochs of the Universe for her honors senior thesis.

\section{PRESS SUMMARY}

Gravitational waves are the ripples of space-time curvature according to Einstein's General Relativity. We search for gravitational waves as binary black holes spiral in towards each other and dissipate energy. We develop the GSTLAL analysis pipeline to match data from the Laser Interferometer Gravitational-wave Observatory (LIGO) with astrophysically modeled templates. Our pipeline evaluation and optimization work will improve the pipeline sensitivity and make it possible to detect gravitational waves from binary black hole coalescence in Advanced LIGO data with high confidence.

\footnotetext{
$\overline{A J U R \text { Volume } 12 \mid \text { Issue } 3 \text { | May } 2015} 103$
} 
Print Edition ISSN 1536-4585

Online Edition ISSN 2375-8732 